\title{
Uncovering Mechanisms of Zanthoxylum piperitum Fruits for the Alleviation of Rheumatoid Arthritis Based on Network Pharmacology
}

\author{
Kikwang Oh ${ }^{(1)}$, Md. Adnan (1) and Dongha Cho* \\ Department of Bio-Health Convergence, College of Biomedical Science, Kangwon National University, \\ Chuncheon 24341, Korea; nivirna07@kangwon.ac.kr (K.O.); mdadnan@kangwon.ac.kr (M.A.) \\ * Correspondence: chodh@kangwon.ac.kr; Tel.: +82-33-250-6475; Fax: +82-33-259-5642
}

check for updates

Citation: Oh, K.; Adnan, M..; Cho, D. Uncovering Mechanisms of Zanthoxylum piperitum Fruits for the Alleviation of Rheumatoid Arthritis Based on Network Pharmacology. Biology 2021, 10, 703. https:// doi.org/10.3390/biology10080703

Academic Editor: Chris G. Dealwis

Received: 14 June 2021

Accepted: 21 July 2021

Published: 23 July 2021

Publisher's Note: MDPI stays neutral with regard to jurisdictional claims in published maps and institutional affiliations.

Copyright: (c) 2021 by the authors. Licensee MDPI, Basel, Switzerland. This article is an open access article distributed under the terms and conditions of the Creative Commons Attribution (CC BY) license (https:// creativecommons.org/licenses/by/ $4.0 /)$.
Simple Summary: The aim of the study is to investigate the bioactives of Zanthoxylum piperitum fruits on rheumatoid arthritis. The methodology to identify the relationship between signaling pathways, targets, and bioactives is based on network pharmacology. The results show that Zanthoxylum piperitum fruits might alleviate inflammatory symptoms of rheumatoid arthritis. Thus, we suggest that Zanthoxylum piperitum fruit is a promising herbal plant to reduce the level of cytokines against rheumatoid arthritis.

Abstract: Zanthoxylum piperitum fruits (ZPFs) have been demonstrated favorable clinical efficacy on rheumatoid arthritis (RA), but its compounds and mechanisms against RA have not been elucidated. This study was to investigate the compounds and mechanisms of ZPFs to alleviate RA via network pharmacology. The compounds from ZPFs were detected by gas chromatography-mass spectrometry (GC-MS) and screened to select drug-likeness compounds through SwissADME. Targets associated with bioactive compounds or RA were identified utilizing bioinformatics databases. The signaling pathways related to RA were constructed; interactions among targets; and signaling pathwaystargets-compounds (STC) were analyzed by RPackage. Finally, a molecular docking test (MDT) was performed to validate affinity between targets and compounds on key signaling pathway(s). GC-MS detected a total of 85 compounds from ZPFs, and drug-likeness properties accepted all compounds. A total of 216 targets associated with compounds 3377 RA targets and 101 targets between them were finally identified. Then, a bubble chart exhibited that inactivation of MAPK (mitogen-activated protein kinase) and activation of PPAR (peroxisome proliferator-activated receptor) signaling pathway might be key pathways against RA. Overall, this work suggests that seven compounds from ZPFs and eight targets might be multiple targets on RA and provide integrated pharmacological evidence to support the clinical efficacy of ZPFs on RA.

Keywords: Zanthoxylum piperitum fruits; rheumatoid arthritis; network pharmacology; MAPK signaling pathway; PPAR signaling pathway

\section{Introduction}

Rheumatoid arthritis (RA) is a long-term systemic autoimmune disorder that deteriorates the synovial joints and is associated with gradual disability [1]. RA is a progressive inflammation caused by joint damage and its functional loss around the articular $[2,3]$. RA can present irrespective of age, diagnosed in around $1 \%$ of the population, brings huge social-economic burden [4]. The main factor causing RA is uncontrollable cytokine secretion due to bone damage; however, the etiology of RA is unknown [5,6]. Commonly, the anti-RA drugs administered are disease-modifying arthritis drugs (DMARDs) and nonsteroidal anti-inflammatory drugs (NSAIDs) in most countries [7]. At present, prolonged administration of these drugs is involved in severe side effects such as upset stomach, nephrotoxicity, and electrolyte imbalance $[8,9]$. In contrast, an animal test demonstrated 
that the repeated dose treatment of plant leaf methanolic extraction with anti-RA efficacy did not alter liver and kidney function [10]. It implies that herbal medicine extracts against RA are better clinical safety than unnatural compounds. For a long time, herbal plants treating RA were essential resources due to their excellent clinical efficacy and low adverse effects [11].

Zanthoxylum piperitum (ZP) belongs to the Rutaceae family, which has been chiefly used as a condiment of food in Korea, Japan, and China [12]. It was reported that essential oil in ZP showed a 38\% reduction of nitric oxide (NO) related to the occurrence and progression of inflammatory joint disease [13]. Another report demonstrated that the peel of ZP has potent anti-inflammatory activities by suppressing nuclear factor kappa-lightchain-enhancer of activated B cells (NF-KB) and caspase- 1 activation in lipopolysaccharide (LPS)-induced RAW264.7 cells [14]. The studies give a hint that ZP might be a herbal medicine to alleviate RA. So far, active compounds and pharmacological mechanisms of ZPFs against RA have not been elucidated. Hence, studies of active compounds and mechanisms of ZPFs against RA should be investigated to prove their therapeutic value.

Network pharmacology is an integrated analytical methodology to understand multiple elements such as compounds, targets and pathways [15]. Network pharmacology can unravel the mechanism of compounds in herbal plants with an integrated concept [16]. Additionally, network pharmacology makes a point of "multiple-targets, multiplecompounds", instead of "one-target, one-compound" [17]. Therefore, network pharmacology is an optimal method to explicate herbal plant issues. Currently, network pharmacology has been utilized to prove bioactive compounds and mechanisms of herbal plants against complex diseases $[18,19]$.

Hence, network pharmacology was utilized to uncover the pharmacological mechanisms of bioactive compounds of ZPFs against RA. Firstly, compounds of ZPFs methanolic extraction identified from GC-MS filtered out drug-likeness candidates on a physicochemical descriptor tool. Secondly, targets related to filtered compounds were retrieved by public bioinformatic databases, and final overlapping targets calculated between compounds and RA targets retrieved by public disease target databases. Thirdly, a key target on proteinprotein interaction (PPI) was identified, and two key signaling pathways of ZPFs against RA were identified by analyzing the final overlapping targets. Then, another key target of signaling pathways related to the occurrence and development of RA was identified by analyzing targets associated with signaling pathways. Finally, a molecular docking test (MDT) was carried out to find po from ZPFs against RA on each target related directly to two key signaling pathways. The workflow diagram is exhibited in Figure 1. 


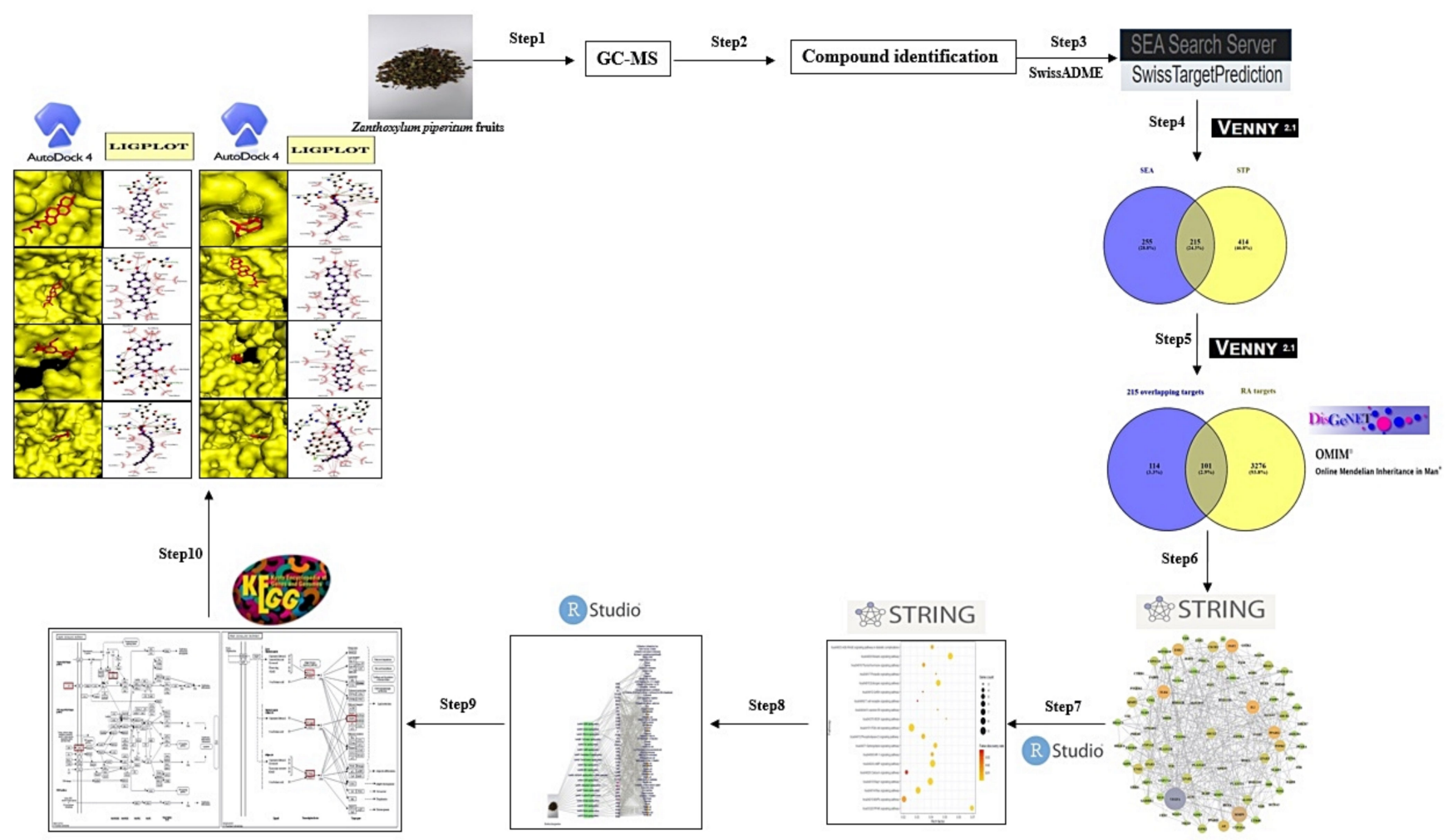

Figure 1. Workflow chart of network pharmacology analysis of ZPFs against RA.

\section{Materials and Methods}

\subsection{Plant Material Collection and Classification}

The Zanthoxylum piperitum fruits (ZPFs) were collected from (latitude: 37.628975 , longitude: 126.742978), Gyeonggi-do, Korea, in August 2020, and the plant was identified by Dr. Dong Ha Cho, Plant biologist and Professor, Department of Bio-Health Convergence, College of Biomedical Science, Kangwon National University. A voucher number (UUC 270) has been stored at Kenaf Corporation in the Department of Bio-Health Convergence, and the material can be used only for research purposes.

\subsection{Plant Preparation, Extraction}

The ZPFs were dried in a shady area at room temperature $\left(20-22{ }^{\circ} \mathrm{C}\right)$ for 21 days, and dried ZPFs made powder using an electric blender. Approximately $50 \mathrm{~g}$ of ZPFs powder was soaked in $800 \mathrm{~mL}$ of 100\% methanol (Daejung, Siheung city, Gyeonggi-do, Korea) for 10 days and repeated three times to achieve a high yield rate. The solvent extract was collected, filtered, and evaporated using a vacuum evaporator (IKA- RV8, Staufen city, Germany). The evaporated sample was dried under a boiling water bath (IKA-HB10, Staufen city, Germany) at $40{ }^{\circ} \mathrm{C}$ for around $8 \mathrm{~h}$ to obtain solid extraction.

\subsection{GC-MS Analysis Condition}

Agilent 7890A was used to carry out GC-MS analysis. GC was equipped with a DB-5 $(30 \mathrm{~m} \times 0.25 \mathrm{~mm} \times 0.25 \mu \mathrm{m})$ capillary column. Initially, the instrument was maintained at a temperature of $100{ }^{\circ} \mathrm{C}$ for $2.1 \mathrm{~min}$. The temperature was rose to $300{ }^{\circ} \mathrm{C}$ at the rate of $25^{\circ} \mathrm{C} / \mathrm{min}$ and maintained for $20 \mathrm{~min}$. Injection port temperature and helium flow rate were ensured as $250{ }^{\circ} \mathrm{C}$ and $1.5 \mathrm{~mL} / \mathrm{min}$, respectively. The ionization voltage was $70 \mathrm{eV}$. The samples injected in split mode at 10:1. The MS scan range was set at 35-900 (m/z). The fragmentation patterns of mass spectra were compared with those stored in the using W8N05ST Library MS database (analyzed 28 April 2021). The percentage of each compound was calculated from the relative peak area of each compound in the chromatogram. 
The concept of integration was used in the ChemStation integrator algorithms (analyzed 28 April 2021) [20].

\subsection{Chemical Compounds Identification and Drug-Likeness Screening}

The chemical compounds from ZPFs were identified through GC-MS analysis. The compounds detected by GC-MS confirmed "drug-likeness" physicochemical property via Lipinski's rule on SwissADME (http:/ /www.swissadme.ch/) (accessed on 14 May 2021). The filtered compounds converted into SMILES (simplified molecular input line entry system) (accessed on 14 May 2021) format through PubChem (https:/ / pubchem.ncbi.nlm. nih.gov/) (accessed on 14 May 2021).

\subsection{Targets Associated with Compounds from ZPFs or Rheumatoid Arthritis}

Targets related to the compounds were identified via both Similarity Ensemble Approach (SEA) (http:/ / sea.bkslab.org/) (accessed on 16 May 2021) [21] and SwissTargetPrediction (STP) (http:/ / www.swisstargetprediction.ch/) (accessed on 16 May 2021) [22] with "Homo Sapiens" mode, both of which is founded on SMILES (accessed on 14 May 2021). The RA-associated targets on humans were retrieved with DisGeNET (https: / www. disgenet.org/) (accessed on 17 May 2021), OMIM (https:/ / www.omim.org/) (accessed on 17 May 2021) and literature. The overlapping targets between compounds of ZPFs and RAassociated targets indicated by VENNY 2.1 (https:/ /bioinfogp.cnb.csic.es/tools/venny/) (accessed on 18 May 2021).

\subsection{PPI Networks and Bubble Chart}

STRING (https://string-db.org/) (accessed on 19 May 2021) [23] was utilized to analyze the PPI network with final overlapping targets. The RPackage was utilized to identify the degree of value. Then, signaling pathways associated with the occurrence and development of RA were visualized on a bubble chart by RPackage; two key signaling pathways with the highest and the lowest rich factor were selected to analyze the relationships against RA.

\subsection{Construction of STC Networks}

The STC networks were utilized to construct a size plot based on the degree of values. In this size map, green rectangles (nodes) represented signaling pathways; gold triangles (nodes) stood for target proteins, and red circles (nodes) stood for compounds; its size represented degree value. The size of gold triangles represented the amount of connectivity with signaling pathways; the size of red circles represented the amount of connectivity with target proteins. The combined networks were constructed by using RPackage (analyzed 20 May 2021).

\subsection{Preparation of Targets for MDT}

Firstly, two targets of MAPK signaling pathway, i.e., FGF2 (PDB ID: 1IIL), VEGFA (PDB ID: 3V2A), and one target of PPAR signaling pathway, i.e., PPARG (PDB ID: 3E00), were identified on STRING via RCSB PDB (https:/ / www.rcsb.org/) (accessed on 21 May 2021). The final three targets selected as .pdb format were converted into .pdbqt format via Autodock (http:/ / autodock.scripps.edu/) (accessed on 21 May 2021).

\subsection{Preparation of Compounds from ZPFs for MDT}

The ligand molecules were converted .sdf from PubChem into .pdb format using Pymol (accessed on 21 May 2021), and the ligand molecules were converted into .pdbqt format through Autodock (accessed on 21 May 2021).

\subsection{Preparation of Positive Standard Ligands for MDT}

The number of two positive ligands on FGF2 antagonists, i.e., NSC172285 (PubChem ID: 299405), NSC37204 (PubChem ID: 235612), and the number of one positive ligand 
on VEGFA antagonist, i.e., BAW2881 (PubChem ID: 16004702), the number of three positive ligands on PPARG antagonists, i.e., Pioglitazone (PubChem ID: 4829), Rosiglitazone (PubChem ID: 77999), Lobeglitazone (PubChem ID: 9826451) were selected to verify the docking score.

\subsection{Ligand-Protein Docking}

The ligand molecules were docked with target proteins utilizing autodock 4 by setting up four energy ranges and eight exhaustiveness as default to obtain 10 different poses of ligand molecules [24]. The 2D binding interactions were used with LigPlot+ v.2.2 (https:/ /www.ebi.ac.uk/thornton-srv/software/LigPlus/) (accessed on 21 May 2021). After docking, ligands of the lowest binding energy (highest affinity) were selected to visualize the ligand-protein interaction in Pymol (Schrödinger, New York, NY, USA).

\subsection{Toxicological Properties Prediction by AdmetSAR}

Toxicological properties of the key bioactive were established using the admetSAR web-service tool (http://lmmd.ecust.edu.cn/admetsar1/predict/) (accessed on 23 May 2021) because toxicity is an essential factor to develop new drugs. Hence, Ames toxicity, carcinogenic properties, acute oral toxicity, and rat acute toxicity were predicted by admetSAR (East China University of Science and Technology, Shanghai, China).

\section{Results}

\subsection{Chemical Compounds from ZPFs}

A total of 85 chemical compounds and its seven key chemical compounds in ZPFs were detected by the GC-MS analysis (Figure 2), and the name of compounds, PubChem ID, retention time (mins), and peak area (\%) were enlisted in Table 1. Lipinski's rules accepted all 85 compounds (molecular weight $\leq 500 \mathrm{~g} / \mathrm{mol}$; Moriguchi octanol-water partition coefficient $\leq 4.15$; number of nitrogen or oxygen $\leq 10$; number of $\mathrm{NH}$ or $\mathrm{OH} \leq 5$ ), and all chemical compounds satisfied with the criteria of "Abbott Bioavailability Score $(>0.1)^{\prime \prime}$ through SwissADME. The TPSA (topological polar surface area) value of chemical compounds was also accepted (Table 2).

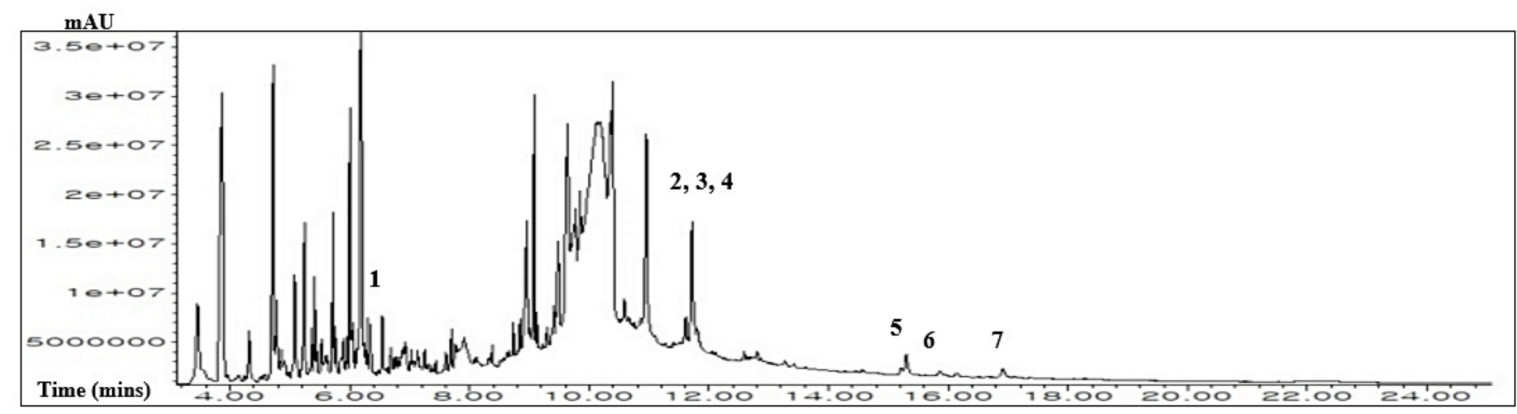

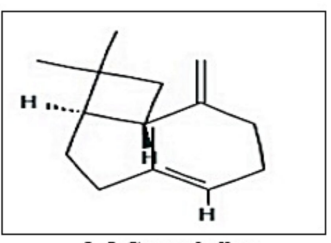

1. $\beta$-Caryophyllene

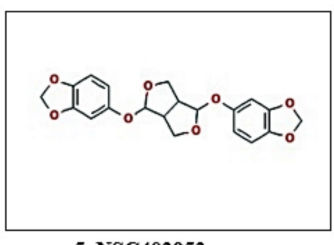

5. NSC402953

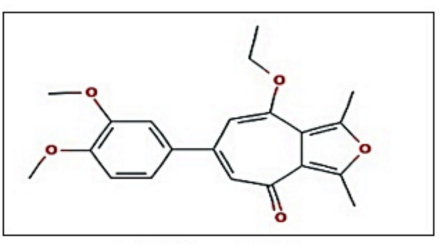

2. CBMicro_013618

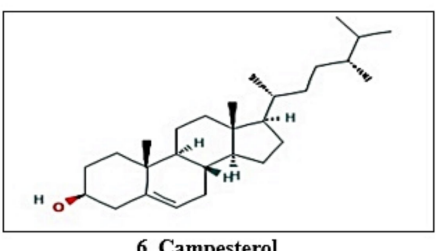

6. Campesterol

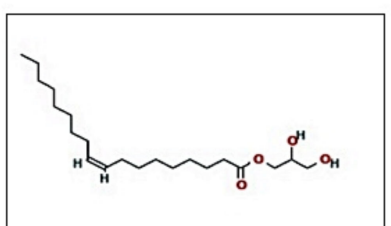

3. Monoolein

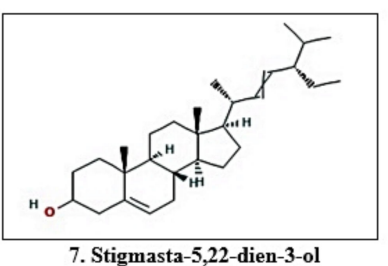

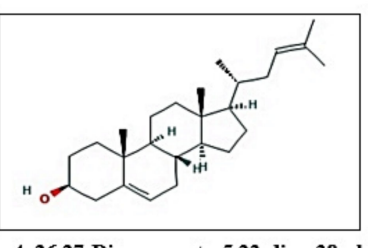

4. 26,27-Dinorergosta-5,23-dien-3ß-ol

Figure 2. A typical GC-MS peak of ZPFs methanolic extract and the number of seven key compounds. 
Table 1. A list of 85 chemical compounds identified from ZPFs via GC-MS and profiling of bioactivities.

\begin{tabular}{|c|c|c|c|c|c|}
\hline No. & Compounds & Pubchem ID & RT (mins) & Area $(\%)$ & $\begin{array}{c}\text { Pharmacological } \\
\text { Activities (Reference) }\end{array}$ \\
\hline 1 & Myrcene & 31253 & 3.462 & 1.49 & $\begin{array}{c}\text { Antibacterial, } \\
\text { Antioxidant, } \\
\text { Fungicide }\end{array}$ \\
\hline 2 & $\begin{array}{l}\text { 3(5)-[[1,2-Dihydroxy-3-propoxy]methyl]-4- } \\
\text { hydroxy-1H-pyrazole-5(3)-carboxamide }\end{array}$ & 135747301 & 3.683 & 0.09 & No reported \\
\hline 3 & $\beta$-Phellandrene & 11142 & 3.866 & 4.28 & Fungicide \\
\hline 4 & Hex-3-yne & 13568 & 3.971 & 0.10 & No reported \\
\hline 5 & 3-Hydroxycyclohexanone & 439950 & 4.087 & 0.06 & No reported \\
\hline 6 & Isopropyl hexanoate & 16832 & 4.145 & 0.12 & No reported \\
\hline 7 & Terpinolene & 11463 & $4.250,4.318$ & 0.59 & $\begin{array}{l}\text { Fungicide, } \\
\text { Antioxidant }\end{array}$ \\
\hline 8 & Vinylcyclooctane & 93331 & 4.520 & 0.07 & No reported \\
\hline 9 & 2-Tetradecynoic acid & 324386 & 4.587 & 0.10 & No reported \\
\hline 10 & Citronellal & 7794 & 4.721 & 2.75 & $\begin{array}{l}\text { Antibacterial, } \\
\text { Fungicide }\end{array}$ \\
\hline 11 & 3-Hydroxy-2,3-dihydromaltol & 119838 & 4.779 & 0.67 & No reported \\
\hline 12 & Pulegol & 92793 & 4.856 & 0.29 & No reported \\
\hline 13 & Octanoic acid & 379 & 4.923 & 0.24 & $\begin{array}{l}\text { Candidicide, } \\
\text { Fungicide }\end{array}$ \\
\hline 14 & (E)-4-Undecenal & 5283357 & 4.981 & 0.10 & No reported \\
\hline 15 & 4-Isopropyl-2-cyclohexenone & 92780 & 5.087 & 1.04 & No reported \\
\hline 16 & Citronellol & 8842 & 5.241 & 1.20 & $\begin{array}{c}\text { Antibacterial, } \\
\text { Candidicide, Sedative }\end{array}$ \\
\hline 17 & (E)-beta-Ocimene & 5281553 & 5.366 & 0.39 & Insecticide \\
\hline 18 & 3,7-Dimethylocta-2,6-dien-1-ol & 4458 & 5.414 & 0.65 & No reported \\
\hline 19 & Spiro[4 .4]nona-1,3-diene, 1,2-dimethyl- & 570800 & 5.452 & 0.21 & No reported \\
\hline 20 & Piperitone & 6987 & 5.529 & 0.41 & Antiasthmatic \\
\hline 21 & Nonanoic acid & 8158 & 5.606 & 0.42 & Perfumery \\
\hline 22 & 8,8-Dimethoxy-2,6-dimethyloct-2-ene & 102507 & 5.721 & 0.98 & No reported \\
\hline 23 & p-Isopropylbenzyl formate & 105515 & 5.760 & 0.40 & No reported \\
\hline 24 & Citronellic acid & 10402 & 5.895 & 0.55 & No reported \\
\hline 25 & $\alpha$-Terpinene & 7462 & 5.952 & 0.24 & Antispasmodic \\
\hline 26 & 2,6-Octadiene, 2,6-dimethyl- & 5365898 & 6.000 & 1.60 & No reported \\
\hline 27 & Terpinyl propionate & 62328 & 6.048 & 0.43 & No reported \\
\hline 28 & Geranyl acetate & 1549026 & 6.193 & 4.60 & Sedative \\
\hline 29 & 3-Methylcyclohexene & 11573 & 6.250 & 0.21 & No reported \\
\hline 30 & $\begin{array}{l}\text { 1,4-Dimethyl-4beta-methoxy-2,5- } \\
\text { cyclohexadien-1 } \alpha \text {-ol }\end{array}$ & 12561656 & 6.298 & 0.33 & No reported \\
\hline 31 & 2-Propenoic acid, 3-phenyl-, methyl ester & 7644 & 6.346 & 0.49 & No reported \\
\hline 32 & 6-Methylenespiro[4.5]decane & 564762 & 6.471 & 0.07 & No reported \\
\hline 33 & $\beta$-Caryophyllene & 5281515 & 6.539 & 0.67 & $\begin{array}{c}\text { Antibacterial, } \\
\text { Antiinflammation }\end{array}$ \\
\hline 34 & Bergamotane & 86000267 & 6.625 & 0.09 & No reported \\
\hline 35 & 3-Methyl-4,7-dioxo-oct-2-enal & 5363705 & 6.693 & 0.30 & No reported \\
\hline 36 & 2,6-Dimethyl-3,5,7-octatriene-2-ol, Z,Z- & 5363692 & 6.779 & 0.24 & No reported \\
\hline 37 & 2-Dodecenoic acid & 5282729 & 6.818 & 0.12 & No reported \\
\hline 38 & 1,6,10-Dodecatrien-3-ol, 3,7,11-trimethyl- & 8888 & 6.885 & 0.35 & No reported \\
\hline 39 & 1-Methyldecahydronaphthalene & 34193 & 6.943 & 0.46 & No reported \\
\hline 40 & Cadina-1(10),4-diene & 10223 & 7.029 & 0.34 & No reported \\
\hline 41 & 2-(4-Methylcyclohexyl)prop-2-en-1-ol & 543946 & 7.135 & 0.42 & No reported \\
\hline 42 & Tetradec-13-enal & 522841 & 7.250 & 0.24 & No reported \\
\hline 43 & 9-Octadecenoic acid & 965 & 7.308 & 0.15 & $\begin{array}{l}\text { Antiinflammation, } \\
\text { Antileukotriene }\end{array}$ \\
\hline 44 & 1,2-Di-but-2-enyl-cyclohexane & 5367574 & 7.375 & 0.10 & No reported \\
\hline
\end{tabular}


Table 1. Cont.

\begin{tabular}{|c|c|c|c|c|c|}
\hline No. & Compounds & Pubchem ID & RT (mins) & Area $(\%)$ & $\begin{array}{c}\text { Pharmacological } \\
\text { Activities (Reference) }\end{array}$ \\
\hline 45 & $\begin{array}{l}\text { 4,12,12-trimethyl-9-methylene-5- } \\
\text { oxatricyclo[8.2.0.04,6]dodecane }\end{array}$ & 73555586 & 7.433 & 0.11 & No reported \\
\hline 46 & 3,4-O-Isopropylidene-d-galactose & 54504880 & 7.568 & 0.08 & No reported \\
\hline 47 & 2-Hexenoic acid, 6-cyclohexyl- & 5367614 & 7.616 & 0.22 & No reported \\
\hline 48 & Heptadec-8-ene & 520230 & 7.693 & 0.35 & No reported \\
\hline 49 & Octane & 356 & 7.779 & 0.35 & No reported \\
\hline 50 & Myristic acid & 11005 & $7.827,8.096$ & 0.44 & $\begin{array}{l}\text { Anticancer, } \\
\text { Antioxidant }\end{array}$ \\
\hline 51 & D-(-)-Kinic Acid & 1064 & 7.914 & 1.35 & No reported \\
\hline 52 & Nonadecanoic acid & 12591 & 8.135 & 0.35 & No reported \\
\hline 53 & 10-Bromoundecanoic acid & 543401 & $8.337,8.385$ & 0.77 & No reported \\
\hline 54 & Stearic acid & 5281 & 8.520 & 0.23 & Hypocholesterolemic \\
\hline 55 & Cysteamine S-sulfate & 76242 & $\begin{array}{l}8.587,9.231 \\
9.298\end{array}$ & 1.27 & No reported \\
\hline 56 & Limonene dioxide & 232703 & 8.635 & 0.23 & No reported \\
\hline 57 & 2,6-Dimethyl-4-nitro-3-phenyl-cyclohexanone & 562366 & 8.664 & 0.26 & No reported \\
\hline 58 & Methyl palmitate & 8181 & 8.731 & 0.46 & No reported \\
\hline 59 & 2,6-Dimethyl-1,3,6-heptatriene & 5368331 & 8.846 & 0.68 & No reported \\
\hline 60 & Palmitic acid & 985 & $8.962,9.020$ & 2.65 & Antioxidant, Pesticide \\
\hline 61 & Neral & 643779 & 9.077 & 1.58 & $\begin{array}{l}\text { Antibacterial, } \\
\text { Antispasmodic }\end{array}$ \\
\hline 62 & 2-Methyl-6-methylene-1,7-octadien-3-one & 93231 & 9.125 & 0.75 & No reported \\
\hline 63 & $\begin{array}{c}\text { Bis(3-benzyl-2,4- } \\
\text { pentanedionato)palladium(II) }\end{array}$ & 5363840 & 9.423 & 1.03 & No reported \\
\hline 64 & Pentamethylbenzenesulfonyl chloride & 590180 & $9.491,9.635$ & 6.52 & No reported \\
\hline 65 & Myrtenal & 61130 & 9.769 & 3.77 & $\begin{array}{l}\text { Antimalarial, } \\
\text { Antiplasmodial }\end{array}$ \\
\hline 66 & N,N-Dimethyl-2-phenylethen-1-amine & 23277871 & $10.154,10.183$ & 20.61 & No reported \\
\hline 67 & Allyl(chloromethyl)dimethylsilane & 556526 & 10.394 & 7.64 & No reported \\
\hline 68 & Cyclohexene, 4-(4-ethylcyclohexyl)-1-pentyl- & 543386 & 10.596 & 1.64 & No reported \\
\hline 69 & 3-Epicycloeucalenol & 543796 & 10.654 & 1.09 & No reported \\
\hline 70 & 2,5-Furandione, 3-dodecenyl- & 5362708 & 10.750 & 0.61 & No reported \\
\hline 71 & 1-cinnamyl-3-methylindole-2-carbaldehyde & $\mathrm{N} / \mathrm{A}$ & 10.875 & 1.35 & No reported \\
\hline 72 & Glyceryl palmitate & 14900 & 10.962 & 4.82 & No reported \\
\hline 73 & 2-Methyl-Z,Z-3,13-octadecadienol & 5364412 & 11.414 & 0.37 & No reported \\
\hline 74 & $\begin{array}{c}\text { Pentadeca-2,3,6,9,12,13-hexaen-8-one, } \\
\text { 2,5,5,11,11,14-hexamethyl- }\end{array}$ & 5370200 & 11.519 & 0.51 & No reported \\
\hline 75 & CBMicro_013618 & 1109374 & 11.616 & 1.04 & No reported \\
\hline 76 & Monoolein & 5283468 & 11.721 & 2.02 & Antioxidant \\
\hline 77 & Cyclohexene, 4-(4-ethylcyclohexyl)-1-pentyl- & 543386 & $11.808,12.596$ & 1.56 & No reported \\
\hline 78 & Cedrane-8,13-diol & 188457 & 12.654 & 0.12 & No reported \\
\hline 79 & 26,27-Dinorergosta-5,23-dien-3 $\beta$-ol & 22213488 & 12.721 & 0.18 & No reported \\
\hline 80 & Cholest-4-en-3-one, 14-methyl- & 277841 & 13.279 & 0.07 & No reported \\
\hline 81 & $(+)$-Sesamolin & 585998 & 15.221 & 0.08 & No reported \\
\hline 82 & NSC402953 & 345349 & 15.308 & 0.32 & No reported \\
\hline 83 & Campesterol & 173183 & 15.866 & 0.12 & $\begin{array}{c}\text { Antioxidant, } \\
\text { Hypocholesterolemic } \\
\text { Antimicrobial, }\end{array}$ \\
\hline 84 & Stigmasta-5,22-dien-3-ol & 53870683 & 16.144 & 0.06 & $\begin{array}{l}\text { Antioxidant, } \\
\text { Antidiabetic } \\
\text { Anticancer, }\end{array}$ \\
\hline 85 & Clionasterol & 457801 & 16.923 & 0.15 & $\begin{array}{l}\text { Antidaibetic, } \\
\text { Antioxidant }\end{array}$ \\
\hline
\end{tabular}


Table 2. Physicochemical properties of chemical compounds for good oral bioavailability and cell membrane permeability.

\begin{tabular}{|c|c|c|c|c|c|c|c|c|}
\hline \multirow{3}{*}{ No. } & \multirow{3}{*}{ Compounds } & \multicolumn{4}{|c|}{ Lipinski Rules } & \multirow{2}{*}{$\begin{array}{l}\text { Lipinski's } \\
\text { Violations }\end{array}$} & \multirow{2}{*}{$\begin{array}{c}\text { Bioavailability } \\
\text { Score }\end{array}$} & \multirow{2}{*}{$\operatorname{TPSA}\left(\AA^{2}\right)$} \\
\hline & & MW & HBA & HBD & MLog P & & & \\
\hline & & $<500$ & $<10$ & $\leq 5$ & $\leq 4.15$ & $\leq 1$ & $>0.1$ & $<140$ \\
\hline 1 & Myrcene & 136.23 & 0 & 0 & 3.56 & 0 & 0.55 & 0.00 \\
\hline 2 & $\begin{array}{l}\text { 3(5)-[[1,2-Dihydroxy-3- } \\
\text { propoxy]methyl]-4-hydroxy-1H- } \\
\text { pyrazole-5(3)-carboxamide }\end{array}$ & 231.21 & 6 & 5 & -2.70 & 0 & 0.55 & 141.69 \\
\hline 3 & $\beta$-Phellandrene & 136.23 & 0 & 0 & 3.27 & 0 & 0.55 & 0.00 \\
\hline 4 & Hex-3-yne & 82.14 & 0 & 0 & 3.37 & 0 & 0.55 & 0.00 \\
\hline 5 & 3-Hydroxycyclohexanone & 114.14 & 2 & 1 & 0.07 & 0 & 0.55 & 37.30 \\
\hline 6 & Isopropyl hexanoate & 158.24 & 2 & 0 & 2.28 & 0 & 0.55 & 26.30 \\
\hline 7 & Terpinolene & 136.23 & 0 & 0 & 3.27 & 0 & 0.55 & 0.00 \\
\hline 8 & Vinylcyclooctane & 138.25 & 0 & 0 & 4.29 & 1 & 0.55 & 0.00 \\
\hline 9 & 2-Tetradecynoic acid & 224.34 & 2 & 1 & 3.58 & 0 & 0.85 & 37.30 \\
\hline 10 & Citronellal & 154.25 & 1 & 0 & 2.59 & 0 & 0.55 & 17.07 \\
\hline 11 & 3-Hydroxy-2,3-dihydromaltol & 144.13 & 4 & 2 & -1.77 & 0 & 0.85 & 66.76 \\
\hline 12 & Pulegol & 154.25 & 1 & 1 & 2.30 & 0 & 0.55 & 20.23 \\
\hline 13 & Octanoic acid & 144.21 & 2 & 1 & 1.96 & 0 & 0.85 & 37.30 \\
\hline 14 & (E)-4-Undecenal & 168.28 & 1 & 0 & 2.88 & 0 & 0.55 & 17.07 \\
\hline 15 & 4-Isopropyl-2-cyclohexenone & 138.21 & 1 & 0 & 1.89 & 0 & 0.55 & 17.07 \\
\hline 16 & Citronellol & 156.27 & 1 & 1 & 2.70 & 0 & 0.55 & 20.23 \\
\hline 17 & (E)-Ocimene & 136.23 & 0 & 0 & 3.56 & 0 & 0.55 & 0.00 \\
\hline 18 & 3,7-Dimethylocta-2,6-dien-1-ol & 154.25 & 1 & 1 & 2.59 & 0 & 0.55 & 20.23 \\
\hline 19 & $\begin{array}{c}\text { Spiro[4.4]nona-1,3-diene, } \\
\text { 1,2-dimethyl- }\end{array}$ & 148.24 & 0 & 0 & 3.56 & 0 & 0.55 & 0.00 \\
\hline 20 & Piperitone & 152.23 & 1 & 0 & 2.20 & 0 & 0.55 & 17.07 \\
\hline 21 & Nonanoic acid & 158.24 & 2 & 1 & 2.28 & 0 & 0.85 & 37.30 \\
\hline 22 & $\begin{array}{c}\text { 8,8-Dimethoxy-2,6-dimethyloct-2- } \\
\text { ene }\end{array}$ & 200.32 & 2 & 0 & 2.75 & 0 & 0.55 & 18.46 \\
\hline 23 & p-Isopropylbenzyl formate & 178.23 & 2 & 0 & 2.58 & 0 & 0.55 & 26.30 \\
\hline 24 & Citronellic acid & 170.25 & 2 & 1 & 2.47 & 0 & 0.85 & 37.30 \\
\hline 25 & alpha-Terpinene & 136.23 & 0 & 0 & 3.27 & 0 & 0.55 & 0.00 \\
\hline 26 & 2,6-Octadiene, 2,6-dimethyl- & 138.25 & 0 & 0 & 3.66 & 0 & 0.55 & 0.00 \\
\hline 27 & Terpinyl propionate & 210.31 & 2 & 0 & 2.92 & 0 & 0.55 & 26.30 \\
\hline 28 & Geranyl acetate & 196.29 & 2 & 0 & 2.95 & 0 & 0.55 & 26.30 \\
\hline 29 & 3-Methylcyclohexene & 96.17 & 0 & 0 & 3.33 & 0 & 0.55 & 0.00 \\
\hline 30 & $\begin{array}{l}\text { 1,4-Dimethyl-4 } \beta \text {-methoxy-2,5- } \\
\text { cyclohexadien- } 1 \alpha \text {-ol }\end{array}$ & 154.21 & 2 & 1 & 0.97 & 0 & 0.55 & 29.46 \\
\hline 31 & $\begin{array}{c}\text { 2-Propenoic acid, 3-phenyl-, methyl } \\
\text { ester }\end{array}$ & 162.19 & 2 & 0 & 2.20 & 0 & 0.55 & 26.30 \\
\hline 32 & 6-Methylenespiro[4.5]decane & 150.26 & 0 & 0 & 4.58 & 1 & 0.55 & 0.00 \\
\hline 33 & beta-Caryophyllene & 204.35 & 0 & 0 & 4.63 & 1 & 0.55 & 0.00 \\
\hline 34 & Bergamotane & 208.38 & 0 & 0 & 5.80 & 1 & 0.55 & 0.00 \\
\hline 35 & 3-Methyl-4,7-dioxo-oct-2-enal & 168.19 & 3 & 0 & 0.29 & 0 & 0.55 & 51.21 \\
\hline 36 & $\begin{array}{c}\text { 2,6-Dimethyl-3,5,7-octatriene-2-ol, } \\
\text { Z,Z- }\end{array}$ & 152.23 & 1 & 1 & 2.49 & 0 & 0.55 & 20.23 \\
\hline 37 & 2-Dodecenoic acid & 198.30 & 2 & 1 & 3.04 & 0 & 0.85 & 37.30 \\
\hline 38 & $\begin{array}{c}\text { 1,6,10-Dodecatrien-3-ol, } \\
\text { 3,7,11-trimethyl- }\end{array}$ & 222.37 & 1 & 1 & 3.86 & 0 & 0.55 & 20.23 \\
\hline 39 & 1-Methyldecahydronaphthalene & 152.28 & 0 & 0 & 4.72 & 1 & 0.55 & 0.00 \\
\hline 40 & Cadina-1(10),4-diene & 204.35 & 1 & 0 & 4.63 & 1 & 0.55 & 0.00 \\
\hline 41 & $\begin{array}{l}\text { 2-(4-Methylcyclohexyl)prop-2-en-1- } \\
\text { ol }\end{array}$ & 154.25 & 1 & 1 & 2.30 & 0 & 0.55 & 20.23 \\
\hline 42 & Tetradec-13-enal & 210.36 & 1 & 0 & 3.70 & 0 & 0.55 & 17.07 \\
\hline 43 & 9-Octadecenoic acid & 282.46 & 2 & 1 & 4.57 & 1 & 0.85 & 37.30 \\
\hline 44 & 1,2-Di-but-2-enyl-cyclohexane & 192.34 & 0 & 0 & 4.37 & 1 & 0.55 & 0.00 \\
\hline
\end{tabular}


Table 2. Cont

\begin{tabular}{|c|c|c|c|c|c|c|c|c|}
\hline \multirow{3}{*}{ No. } & \multirow{3}{*}{ Compounds } & \multicolumn{4}{|c|}{ Lipinski Rules } & \multirow{2}{*}{$\begin{array}{l}\text { Lipinski's } \\
\text { Violations }\end{array}$} & \multirow{2}{*}{$\begin{array}{l}\text { Bioavailability } \\
\text { Score }\end{array}$} & \multirow{2}{*}{$\operatorname{TPSA}\left(\AA^{2}\right)$} \\
\hline & & MW & HBA & HBD & MLog P & & & \\
\hline & & $<500$ & $<10$ & $\leq 5$ & $\leq 4.15$ & $\leq 1$ & $>0.1$ & $<140$ \\
\hline 45 & $\begin{array}{l}\text { 4,12,12-trimethyl-9-methylene-5- } \\
\text { oxatricyclo[8.2.0.04,6]dodecane }\end{array}$ & 220.35 & 1 & 0 & 3.67 & 0 & 0.55 & 12.53 \\
\hline 46 & 3,4-O-Isopropylidene-d-galactose & 220.22 & 6 & 3 & -1.34 & 0 & 0.55 & 88.38 \\
\hline 47 & 2-Hexenoic acid, 6-cyclohexyl- & 196.29 & 2 & 1 & 2.65 & 0 & 0.85 & 37.30 \\
\hline 48 & Heptadec-8-ene & 238.45 & 0 & 0 & 6.54 & 1 & 0.55 & 0.00 \\
\hline 49 & Octane & 114.23 & 0 & 0 & 4.20 & 1 & 0.55 & 0.00 \\
\hline 50 & Myristic acid & 228.37 & 2 & 1 & 3.69 & 0 & 0.85 & 37.30 \\
\hline 51 & D-(-)-Kinic Acid & 192.17 & 6 & 5 & -2.14 & 0 & 0.55 & 118.22 \\
\hline 52 & Nonadecanoic acid & 298.50 & 2 & 1 & 4.91 & 1 & 0.85 & 37.30 \\
\hline 53 & 10-Bromoundecanoic acid & 265.19 & 2 & 1 & 3.29 & 0 & 0.85 & 37.30 \\
\hline 54 & Stearic acid & 284.48 & 2 & 1 & 4.67 & 1 & 0.85 & 37.30 \\
\hline 55 & Cysteamine S-sulfate & 157.21 & 4 & 2 & -1.51 & 0 & 0.55 & 114.07 \\
\hline 56 & Limonene dioxide & 168.23 & 2 & 0 & 1.52 & 0 & 0.55 & 25.06 \\
\hline 57 & $\begin{array}{l}\text { 2,6-Dimethyl-4-nitro-3-phenyl- } \\
\text { cyclohexanone }\end{array}$ & 247.29 & 3 & 0 & 1.66 & 0 & 0.55 & 62.89 \\
\hline 58 & Methyl palmitate & 270.45 & 2 & 0 & 4.44 & 1 & 0.55 & 26.30 \\
\hline 59 & 2,6-Dimethyl-1,3,6-heptatriene & 122.21 & 0 & 0 & 3.26 & 0 & 0.55 & 0.00 \\
\hline 60 & Palmitic acid & 256.42 & 2 & 1 & 4.19 & 1 & 0.85 & 37.30 \\
\hline 61 & Neral & 152.23 & 1 & 0 & 2.49 & 0 & 0.55 & 17.07 \\
\hline 62 & $\begin{array}{l}\text { 2-Methyl-6-methylene-1,7- } \\
\text { octadien-3-one }\end{array}$ & 150.22 & 1 & 0 & 2.40 & 0 & 0.55 & 17.07 \\
\hline 63 & $\begin{array}{c}\text { Bis(3-benzyl-2,4- } \\
\text { pentanedionato)palladium(II) }\end{array}$ & 486.90 & 4 & 2 & 2.69 & 0 & 0.85 & 74.60 \\
\hline 64 & $\begin{array}{l}\text { Pentamethylbenzenesulfonyl } \\
\text { chloride }\end{array}$ & 246.75 & 2 & 0 & 3.04 & 0 & 0.55 & 42.52 \\
\hline 65 & Myrtenal & 150.22 & 1 & 0 & 2.20 & 0 & 0.55 & 17.07 \\
\hline 66 & $\begin{array}{l}\text { N,N-Dimethyl-2-phenylethen-1- } \\
\text { amine }\end{array}$ & 147.22 & 0 & 0 & 2.40 & 0 & 0.55 & 3.24 \\
\hline 67 & Allyl(chloromethyl)dimethylsilane & 148.71 & 0 & 0 & 2.81 & 0 & 0.55 & 0.00 \\
\hline 68 & $\begin{array}{c}\text { Cyclohexene, } \\
\text { 4-(4-ethylcyclohexyl)-1-pentyl- }\end{array}$ & 262.47 & 0 & 0 & 6.61 & 1 & 0.55 & 0.00 \\
\hline 69 & 3-Epicycloeucalenol & 426.72 & 1 & 1 & 6.92 & 1 & 0.55 & 20.23 \\
\hline 70 & 2,5-Furandione, 3-dodecenyl- & 266.38 & 3 & 0 & 3.53 & 0 & 0.55 & 43.37 \\
\hline 71 & $\begin{array}{l}\text { 1-cinnamyl-3-methylindole-2- } \\
\text { carbaldehyde }\end{array}$ & 275.34 & 1 & 0 & 3.20 & 0 & 0.55 & 22.00 \\
\hline 72 & Glyceryl palmitate & 330.50 & 4 & 2 & 3.18 & 0 & 0.55 & 66.76 \\
\hline 73 & $\begin{array}{l}\text { 2-Methyl-Z,Z-3,13-octadecadienol } \\
\text { Pentadeca-2,3,6,9,12,13-hexaen-8- }\end{array}$ & 280.49 & 1 & 1 & 4.91 & 1 & 0.55 & 20.23 \\
\hline 74 & $\begin{array}{l}\text { one, } \\
2,5,5,11,11,14 \text {-hexamethyl- }\end{array}$ & 298.46 & 1 & 0 & 4.93 & 1 & 0.55 & 17.07 \\
\hline 75 & CBMicro_013618 & 354.40 & 5 & 0 & 1.66 & 0 & 0.55 & 57.90 \\
\hline 76 & Monoolein & 356.54 & 4 & 2 & 3.52 & 0 & 0.55 & 66.76 \\
\hline 77 & $\begin{array}{c}\text { Cyclohexene, } \\
\text { 4-(4-ethylcyclohexyl)-1-pentyl- }\end{array}$ & 262.47 & 0 & 0 & 6.61 & 1 & 0.55 & 0.00 \\
\hline 78 & Cedrane-8,13-diol & 238.37 & 2 & 2 & 2.88 & 0 & 0.55 & 40.46 \\
\hline 79 & 26,27 -Dinorergosta-5,23-dien-3 $\beta$-ol & 370.61 & 1 & 1 & 6.03 & 1 & 0.55 & 20.23 \\
\hline 80 & Cholest-4-en-3-one, 14-methyl- & 398.66 & 1 & 0 & 6.43 & 1 & 0.55 & 17.07 \\
\hline 81 & $(+)$-Sesamolin & 370.35 & 7 & 0 & 1.85 & 0 & 0.55 & 64.61 \\
\hline 82 & NSC402953 & 386.35 & 8 & 0 & 1.74 & 0 & 0.55 & 73.84 \\
\hline 83 & Campesterol & 400.68 & 1 & 1 & 6.54 & 1 & 0.55 & 20.23 \\
\hline 84 & Stigmasta-5,22-dien-3-ol & 412.69 & 1 & 1 & 6.62 & 1 & 0.55 & 20.23 \\
\hline 85 & Clionasterol & 414.71 & 1 & 1 & 6.73 & 1 & 0.55 & 20.23 \\
\hline
\end{tabular}




\subsection{Overlapping Targets between SEA and STP Related to Chemical Compounds}

A total of 470 targets from Similarity Ensemble Approach (SEA) and 629 targets from SwissTargetPrediction (STP) were associated with 85 chemical compounds (Supplementary Table S1). The Venn diagram showed that 215 targets overlapped between the two public databases (Supplementary Table S1) (Figure 3A).

(A)

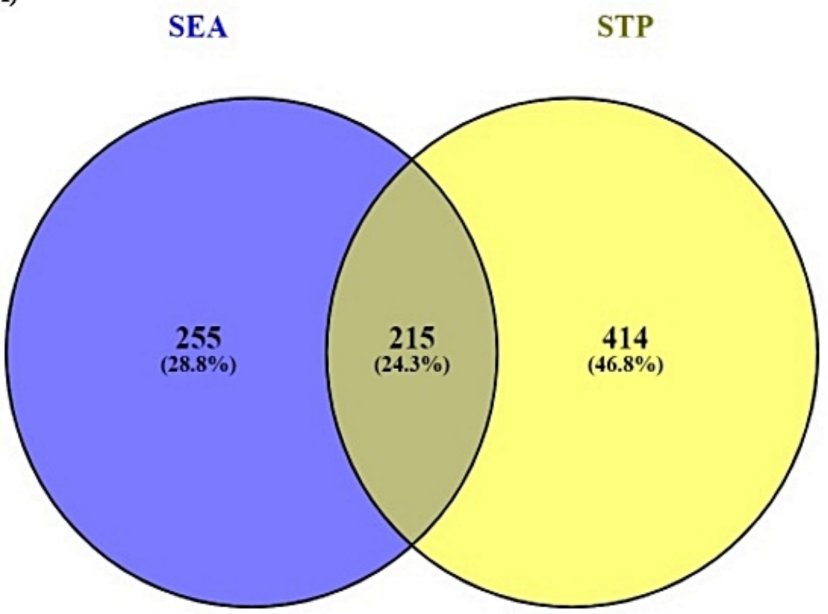

(B)

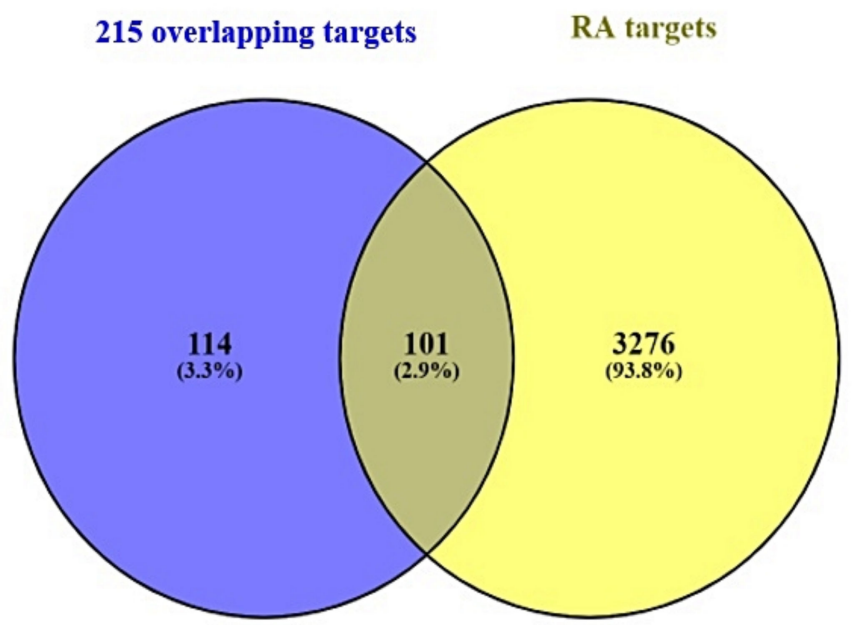

Figure 3. (A) The number of 215 overlapping targets between SEA (470 targets) and STP (629 targets). (B) The number of 101 final overlapping targets between 215 overlapping targets from two databases (SEA and STP) and RA associated with targets (3377 targets).

\subsection{Overlapping Targets between RA-Related Genes and the Final 101 Overlapping Targets}

A total of 3377 targets associated with RA were selected by retrieving from DisGeNET, Online Mendelian Inheritance in Man (OMIM) databases and literature (Supplementary Table S2). Venn diagram's result displayed 101 overlapping targets that were selected between 3377 targets related to RA and the 215 overlapping targets (Figure 3B) (Supplementary Table S3).

\subsection{Acquisition of a Key Target from PPI Networks}

From STRING analysis, 99 out of 101 overlapping targets were directly associated with RA occurrence and development, indicating 99 nodes and 469 edges (Figure 4). The two removed targets (CA1 and CA3) had no connectivity to the overlapping 101 targets. In PPI networks, Vascular Endothelial Growth Factor A (VEGFA) was the highest degree (42) and is considered a key target (Table 3).

Table 3. The degree value of target in PPI.

\begin{tabular}{cccccc}
\hline No. & Target & Degree of Value & No. & Target & Degree of Value \\
\hline 1 & VEGFA & 42 & 51 & ENPP2 & 9 \\
2 & MMP9 & 28 & 52 & PPARA & 8 \\
3 & TLR4 & 23 & 53 & PLA2G2A & 8 \\
4 & IL2 & 23 & 54 & NLRP3 & 8 \\
5 & FGF2 & 23 & 55 & MAOA & 8 \\
6 & PPARG & 22 & 56 & F3 & 8 \\
7 & ESR1 & 21 & 57 & EDNRA & 8 \\
8 & PTPRC & 19 & 58 & AKR1B1 & 8 \\
9 & AR & 19 & 59 & SHBG & 7 \\
10 & CXCR3 & 18 & 60 & PTPN6 & 7 \\
11 & MMP2 & 17 & 61 & PRKCA & 7 \\
12 & CNR1 & 17 & 62 & DHFR & 7 \\
\hline
\end{tabular}


Table 3. Cont.

\begin{tabular}{|c|c|c|c|c|c|}
\hline No. & Target & Degree of Value & No. & Target & Degree of Value \\
\hline 13 & LPAR3 & 16 & 63 & TYR & 6 \\
\hline 14 & ABCG2 & 16 & 64 & NR1I3 & 6 \\
\hline 15 & NR3C1 & 15 & 65 & MAOB & 6 \\
\hline 16 & LPAR2 & 15 & 66 & HMGCR & 6 \\
\hline 17 & LPAR1 & 15 & 67 & HDAC6 & 6 \\
\hline 18 & HDAC1 & 15 & 68 & ESR2 & 6 \\
\hline 19 & $\mathrm{ABCB} 1$ & 14 & 69 & RORC & 5 \\
\hline 20 & S1PR1 & 14 & 70 & HSD11B1 & 5 \\
\hline 21 & CYP2C19 & 14 & 71 & GSR & 5 \\
\hline 22 & CYP1A2 & 14 & 72 & FGF1 & 5 \\
\hline 23 & CYP19A1 & 14 & 73 & $\mathrm{BCHE}$ & 5 \\
\hline 24 & ALOX5 & 14 & 74 & RARB & 4 \\
\hline 25 & ABCB1 & 14 & 75 & HSD11B2 & 4 \\
\hline 26 & PLA2G1B & 13 & 76 & GRK6 & 4 \\
\hline 27 & TNFRSF1A & 12 & 77 & GPR35 & 4 \\
\hline 28 & PLA2G4A & 12 & 78 & GPBAR1 & 4 \\
\hline 29 & GABBR2 & 12 & 79 & DHCR7 & 4 \\
\hline 30 & GABBR1 & 12 & 80 & PTGER2 & 3 \\
\hline 31 & CNR2 & 12 & 81 & PPARD & 3 \\
\hline 32 & PTGES & 11 & 82 & PDE4B & 3 \\
\hline 33 & PTGER4 & 11 & 83 & IL6ST & 3 \\
\hline 34 & NOS2 & 11 & 84 & HEXB & 3 \\
\hline 35 & MTNR1B & 11 & 85 & CES2 & 3 \\
\hline 36 & LTB4R & 11 & 86 & AKR1B10 & 3 \\
\hline 37 & GLI1 & 11 & 87 & TTR & 2 \\
\hline 38 & EDNRB & 11 & 88 & RORA & 2 \\
\hline 39 & TLR9 & 10 & 89 & KCNA3 & 2 \\
\hline 40 & S1PR3 & 10 & 90 & FABP3 & 2 \\
\hline 41 & MMP1 & 10 & 91 & CTRB1 & 2 \\
\hline 42 & HDAC3 & 10 & 92 & CPA1 & 2 \\
\hline 43 & G6PD & 10 & 93 & CA2 & 2 \\
\hline 44 & CYP17A1 & 10 & 94 & ASAH1 & 2 \\
\hline 45 & ALOX12 & 10 & 95 & $\mathrm{ACP} 1$ & 2 \\
\hline 46 & ALDH1A1 & 10 & 96 & PDE4D & 1 \\
\hline 47 & VDR & 9 & 97 & PAM & 1 \\
\hline 48 & TRPV1 & 9 & 98 & HEXA & 1 \\
\hline 49 & SLC6A4 & 9 & 99 & GSTK1 & 1 \\
\hline 50 & $\mathrm{SHH}$ & 9 & & & \\
\hline
\end{tabular}

\subsection{Identification of Two Key Signaling Pathways from a Bubble Chart}

The results of Kyoto Encyclopedia of Genes and Genomes (KEGG) pathway enrichment analysis unveiled that 19 signaling pathways were connected with 40 out of 101 targets (false discovery rate $<0.05$ ). The 19 signaling pathways were directly related to RA, indicating that these 19 signaling pathways might be significant pathways of ZPFs against RA. The description of 19 signaling pathways were shown in Table 4. Additionally, a bubble chart indicated that inactivation of MAPK signaling pathway and activation of PPAR signaling pathway might be key signaling pathways of ZPFs to alleviate RA (Figure 5). Specifically, MAPK signaling pathway is associated with VEGFA (a hub target) in holistic PPI networks while PPAR signaling pathway is not related to VEGFA. 


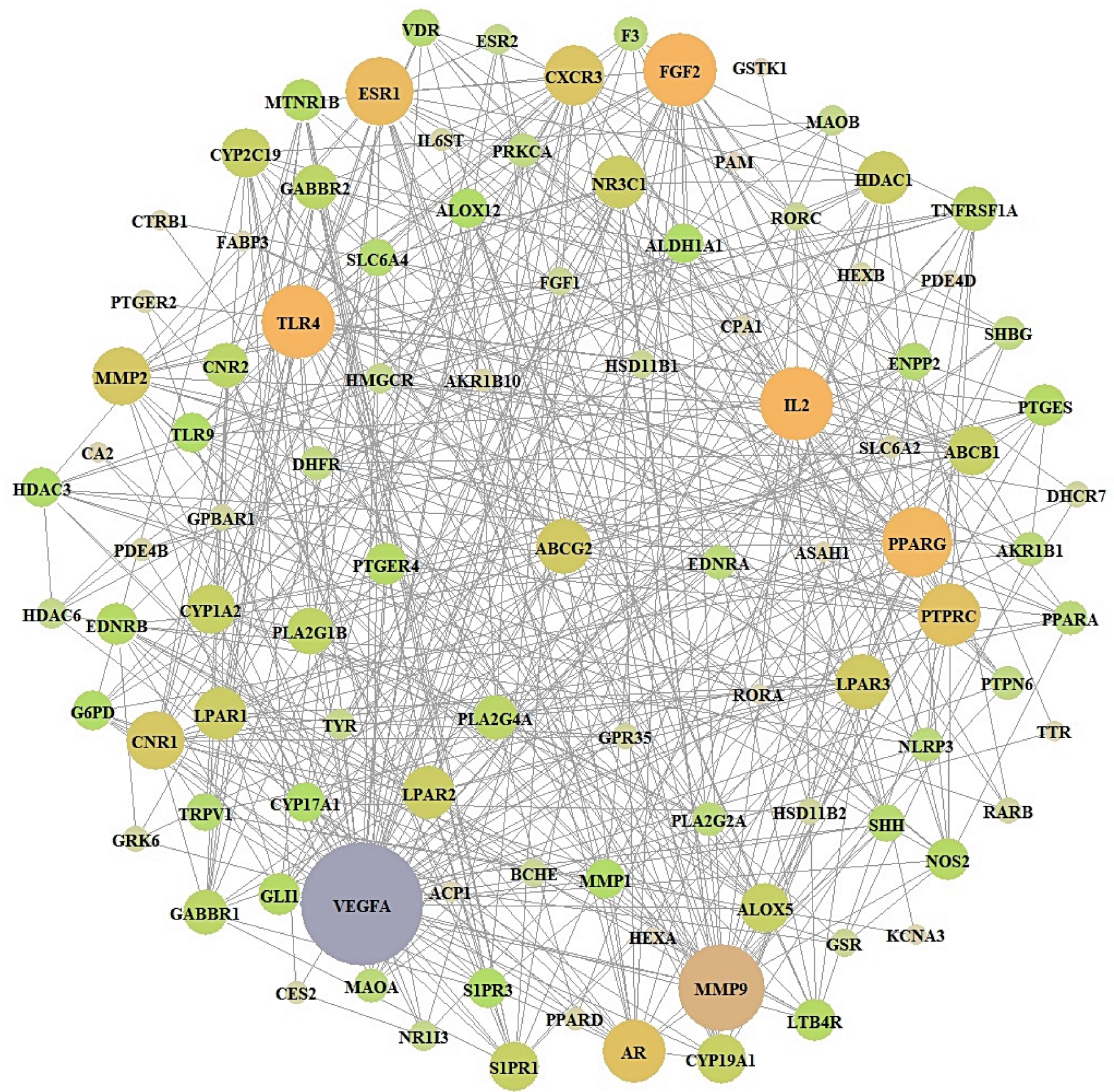

Figure 4. PPI networks (99 nodes, 469 edges). The size of the circle: degree of values.

Table 4. Targets in 19 signaling pathways associated with RA.

\begin{tabular}{ccc}
\hline KEGG ID \& Description & Target Genes & False Discovery Rate \\
\hline hsa04933:AGE-RAGE signaling pathway in & PRKCA,MMP2,VEGFA,F3 & 0.01010 \\
diabetic complications & PRKCA,VEGFA,EDNRB,MMP1,MMP2,MMP9,NOS2 & 0.00018 \\
hsa04926:Relaxin signaling pathway & PRKCA,ESR1,HDAC1,HDAC3 & 0.01460 \\
hsa04919:Thyroid hormone & ESR1,ESR2,CYP17A1 & 0.02200 \\
signaling pathway & GABBR1,GABBR2,MMP2,MMP9,ESR1,ESR2 & 0.00110 \\
hsa04917:Prolactin signaling pathway & PRKCA,MMP2,PLA2G4A & 0.03510 \\
hsa04915:Estrogen signaling pathway & & \\
hsa04912:GnRH signaling pathway &
\end{tabular}


Table 4. Cont.

\begin{tabular}{ccc}
\hline KEGG ID \& Description & Target Genes & False Discovery Rate \\
\hline hsa04660:T cell receptor signaling pathway & IL2,PTPRC,PTPN6 & 0.03950 \\
hsa04644:Fc epsilon RI signaling pathway & PRKCA,ALOX5,PLA2G4A & 0.02070 \\
hsa04370:VEGF signaling pathway & VEGFA,PRKCA,PLA2G4A & 0.01690 \\
hsa04151:PI3K-Akt signaling pathway & PRKCA,VEGFA,IL2,TLR4,FGF1,FGF2,LPAR1, & 0.00110 \\
hsa04072:Phospholipase D & LPAR2,LPAR3 & 0.00660 \\
signaling pathway & PRKCA,MMP2,LPAR1,LPAR2,LPAR3 & 0.00340 \\
hsa04071:Sphingolipid signaling pathway & PRKCA,S1PR1,S1PR3,ASAH1,TNFRSF1A & 0.01010 \\
hsa04066:HIF-1 signaling pathway & PRKCA,VEGFA,TLR4,NOS2 & 0.00023 \\
hsa04024:cAMP signaling pathway & PPARA,EDNRA,PDE4B,PDE4D,GABBR1,GABBR2, & 0.03850 \\
hsa04020:Calcium signaling pathway & PTGER2,GLI1 & 0.00025 \\
hsa04015:Rap1 signaling pathway & PRKCA,VEGFA,FGF1,FGF2,CNR1,LPAR1,LPAR2,LPAR3 & 0.00200 \\
hsa04014:Ras signaling pathway & PRKCA,VEGFA,FGF1,FGF2,PLA2G2A,PLA2G1B & 0.01810 \\
hsa04010:MAPK signaling pathway & PRKCA,VEGFA,FGF1,FGF2,TNFRSF1A,PLA2G4A & 0.00070 \\
hsa03320:PPAR signaling pathway & PPARA,PPARD,PPARG,FABP3,MMP1 &
\end{tabular}

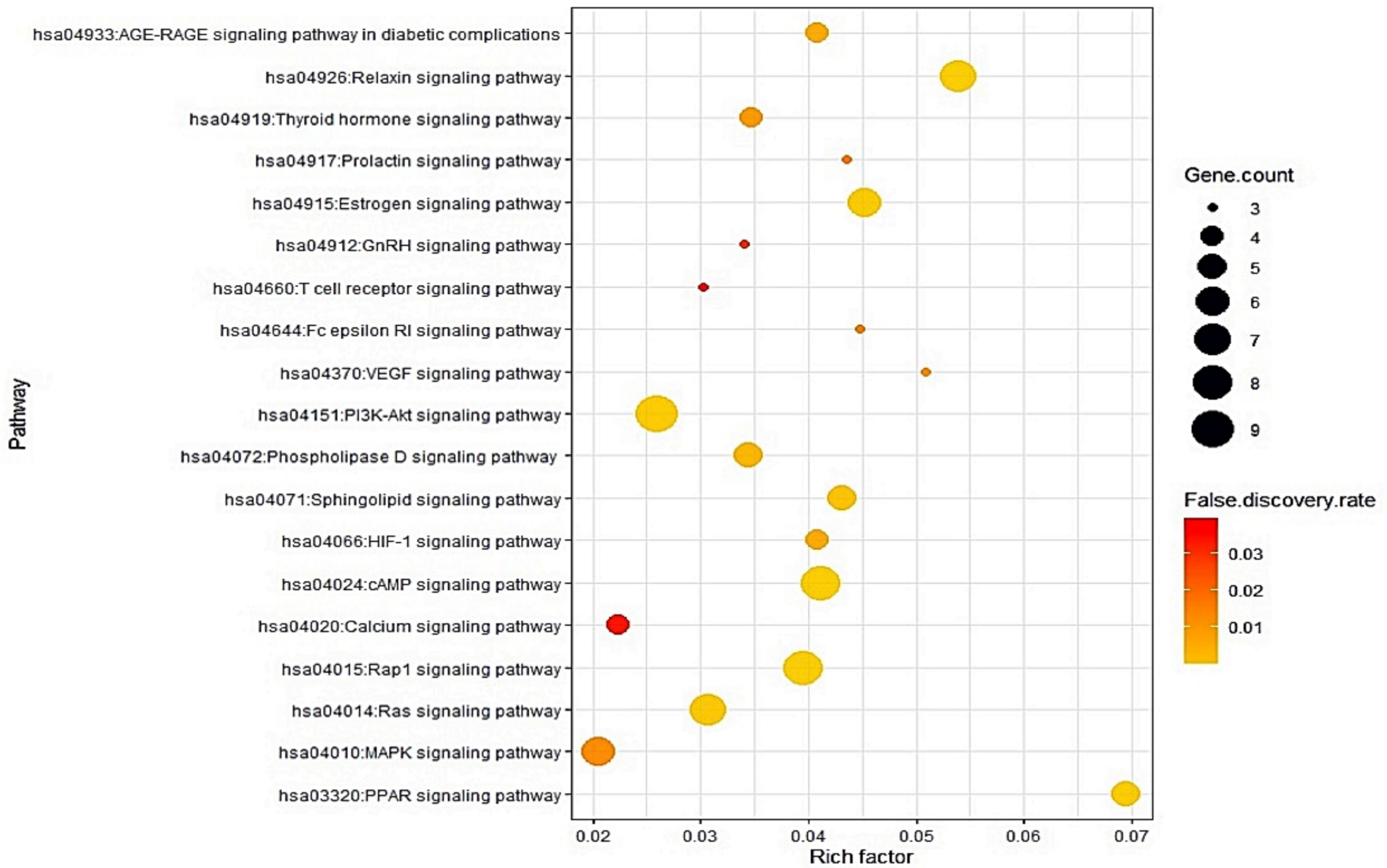

Figure 5. Bubble chart of 19 signaling pathways associated with cancer.

\subsection{Construction of Signaling Pathway-Target-Compound Network}

The signaling pathway-target-compound (STC) network is displayed in Figure 6. There were 19 pathways, 40 targets, and 63 compounds (122 nodes, 488 edges). The nodes stood for the total number of relationships between signaling pathways, targets, and compounds. The edges represented the relationship of three elements (19 pathways, 40 targets, and 63 compounds). The STC network suggested that the uppermost target is Protein Kinase C Alpha (PRKCA) with 14 degrees among 19 signaling pathways (Table 5). 


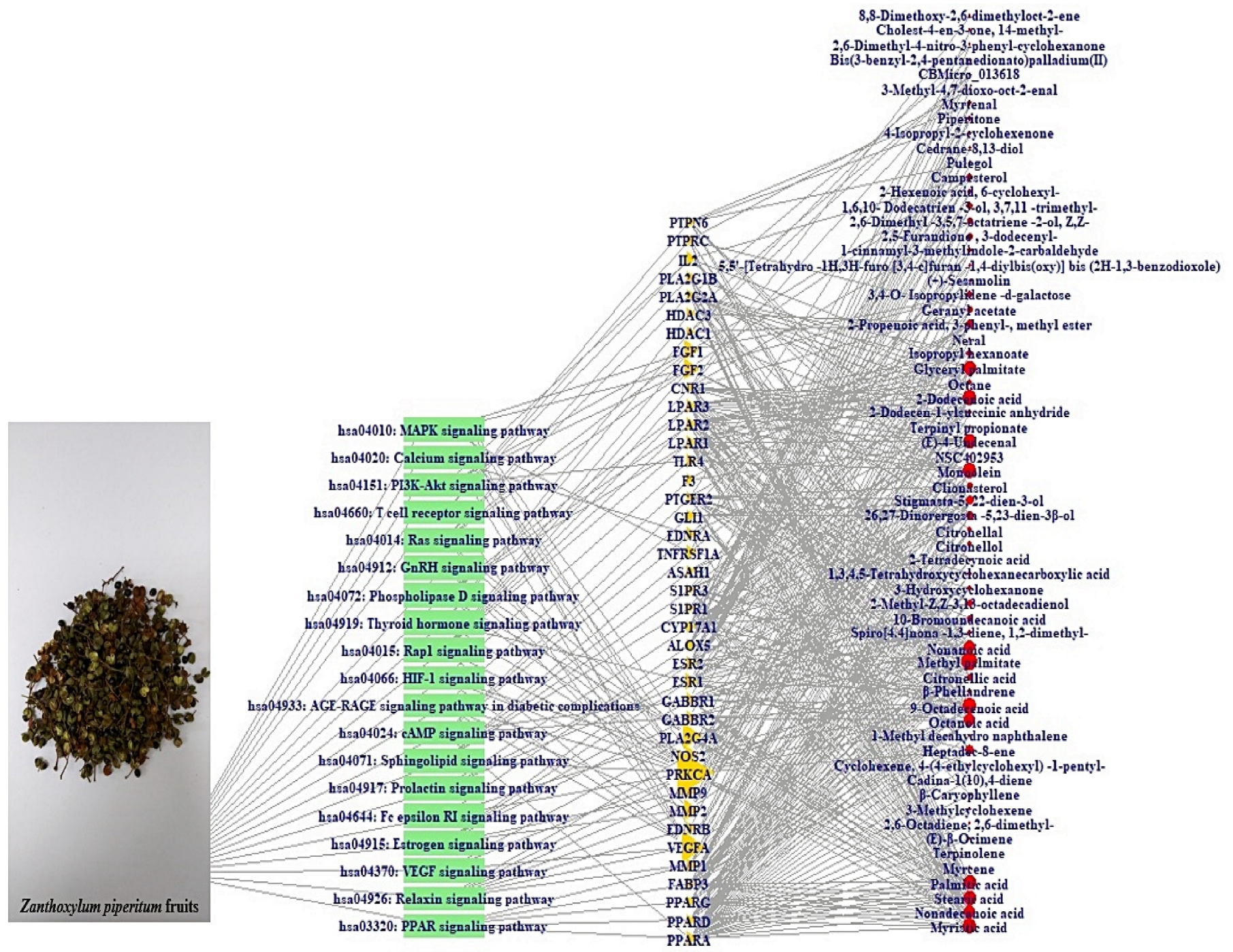

Figure 6. STC networks (122 nodes, 488 edges). Green rectangle: signaling pathway; gold triangle: targets; red circle: compounds.

Table 5. The degree value of target in STC.

\begin{tabular}{cccccc}
\hline No. & Target & Degree of Value & No. & Target & Degree of Value \\
\hline 1 & PRKCA & 14 & 21 & TLR4 & 2 \\
2 & VEGFA & 8 & 22 & IL2 & 2 \\
3 & MMP2 & 5 & 23 & PPARD & 1 \\
4 & PLA2G4A & 4 & 24 & PPARG & 1 \\
5 & FGF2 & 4 & 25 & FABP3 & 1 \\
\hline 6 & FGF1 & 4 & 26 & ALOX5 & 1 \\
7 & NOS2 & 3 & 27 & CYP17A1 & 1 \\
8 & ESR1 & 3 & 28 & S1PR1 & 1 \\
9 & LPAR1 & 3 & 29 & S1PR3 & 1 \\
10 & LPAR2 & 3 & 30 & ASAH1 & 1 \\
11 & LPAR3 & 3 & 31 & GLI1 & 1 \\
12 & PPARA & 2 & 32 & PTGER2 & 1 \\
13 & MMP1 & 2 & 33 & F3 & 1 \\
14 & EDNRB & 2 & 34 & CNR1 & 1 \\
15 & MMP9 & 2 & 35 & HDAC1 & 1 \\
16 & GABBR2 & 2 & 36 & HDAC3 & 1 \\
17 & GABBR1 & 2 & 37 & PLA2G2A & 1 \\
\hline
\end{tabular}


Table 5. Cont.

\begin{tabular}{cccccc}
\hline No. & Target & Degree of Value & No. & Target & Degree of Value \\
\hline 18 & ESR2 & 2 & 38 & PLA2G1B & 1 \\
19 & TNFRSF1A & 2 & 39 & PTPRC & 1 \\
20 & EDNRA & 2 & 40 & PTPN6 & 1 \\
\hline
\end{tabular}

\subsection{KEGG Pathway Enrichment Analysis}

KEGG pathway enrichment analysis [25] reveals that 19 signaling pathways are associated with the occurrence and development of RA. Out of 19 signaling pathways, MAPK signaling pathway and PPAR signaling pathway were most significantly related to RA (Figure 7). The red rectangles indicated core targets on the two signaling pathways. One of the MAPK signaling pathways, PRKCA (a species of PKC), is an excellent immunomodulatory target, which is linked to the onset of inflammatory arthritis, including RA [26]. It was subsequently shown that inactivation of PRKCA results in the inhibition of c-fos; eventually, the cascade leads to the amelioration of RA in an animal model [27]. One of the PPAR signaling pathways, FABP3, is located in the upstream region to regulate lipid metabolism. It was reported that defective lipid metabolism was observed in RA patients who are persistent to proinflammatory responses [28].

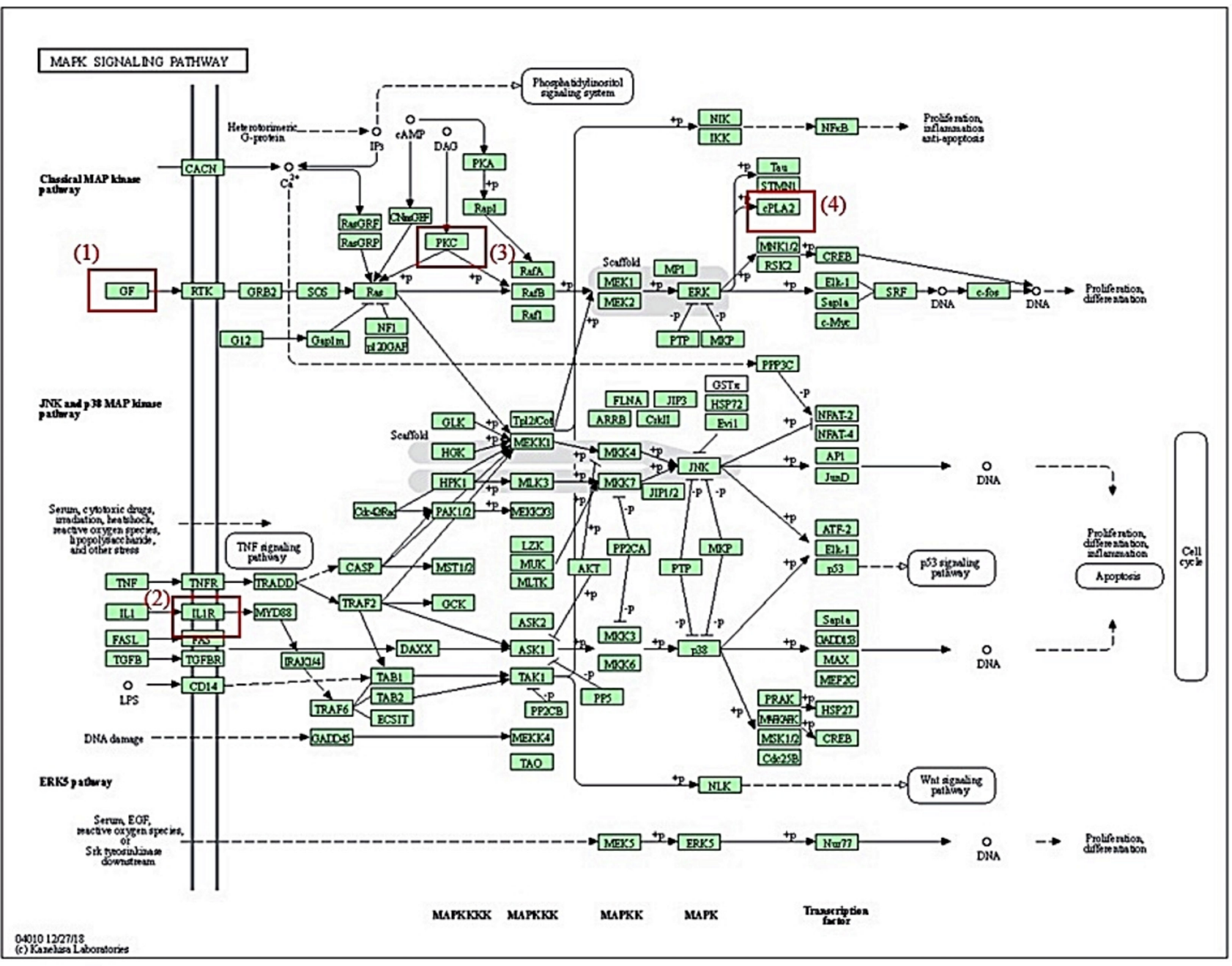

(A)

Figure 7. Cont. 


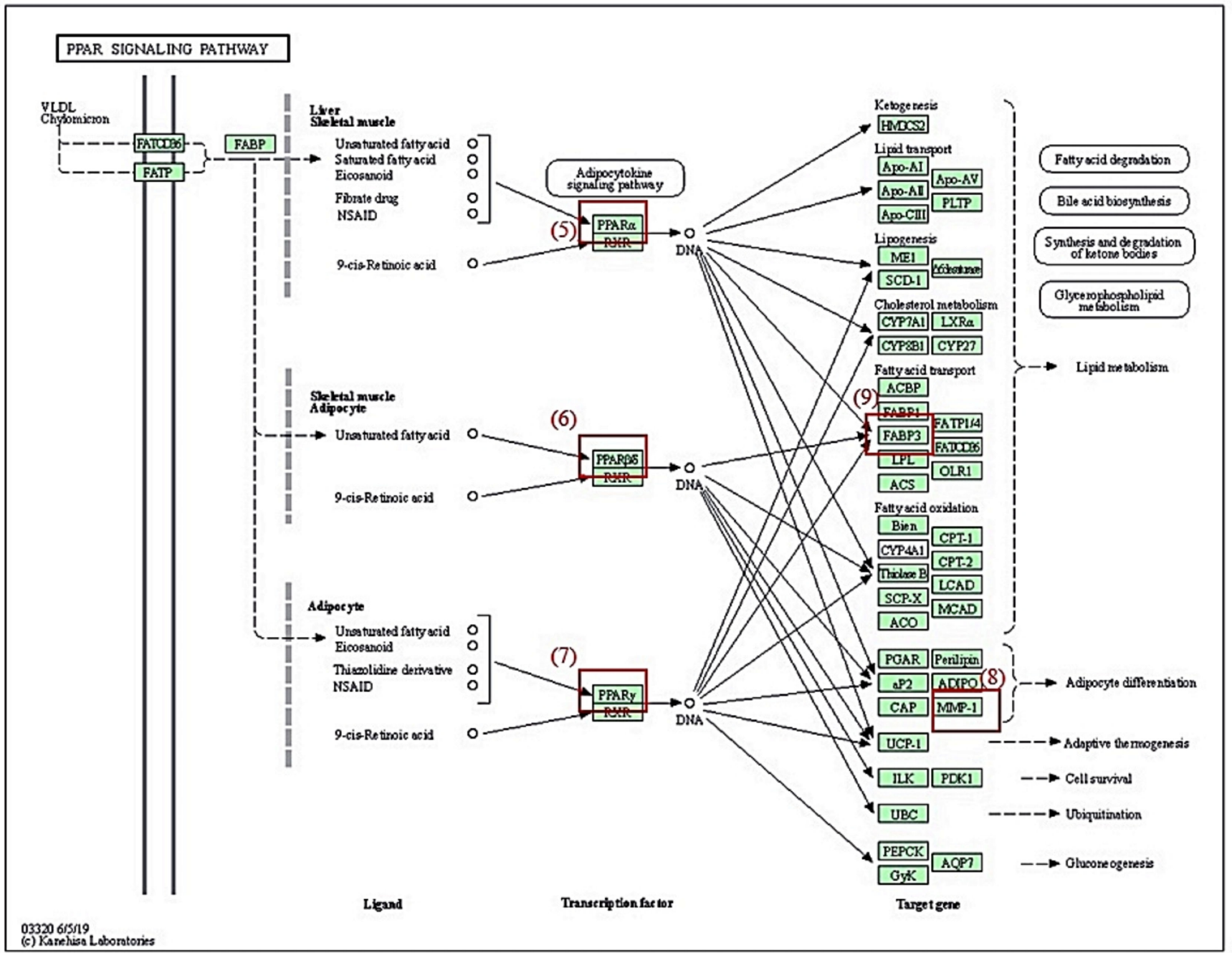

(B)

Figure 7. KEGG pathway enrichment map. (A) MAPK signaling pathway. (B) PPAR signaling pathway. Red rectangles represent key targets: (1) FGF1, FGF2, VEGFA; (2) TNFRSF1A; (3) PRKCA; (4) PLA2G4A; (5) PPARA; (6) PPARD; (7) PPARG; (8) MMP1; (9) FABP3.

3.8. MDT of 6 Targets and 23 Chemical Compounds Associated with MAPK Signaling Pathway

From both SEA and STP databases, it was uncovered that FGF1 is associated with two chemical compounds, FGF2 with four chemical compounds, VEGFA with five chemical compounds, TNFRSF1A with two chemical compounds, PRKCA with 16 chemical compounds, and PLA2G4A with four chemical compounds. Out of 33 chemical compounds, 10 chemical compounds overlapped, and finally, 23 chemical compounds were identified on the MAPK signaling pathway.

The MDT performed to evaluate affinity between target and ligand displayed the greatest affinity complex, depicted in Figure 8 . In detail, campesterol $(-8.4 \mathrm{kcal} / \mathrm{mol})$ docked on the FGF1 target (PDB ID: 3OJ2) had the greatest affinity; however, it had a lower affinity than suramin sodium $(-19.1 \mathrm{kcal})$ as a positive control. The 26,27-Dinorergosta5,23-dien-3 $\beta$-ol $(-8.0 \mathrm{kcal} / \mathrm{mol})$ docked on FGF2 target (PDB ID: 1IIL) had the highest affinity; its affinity was lower than NSC172285 $(-14.7 \mathrm{kcal} / \mathrm{mol})$ as a positive control. The 3,4-O-Isopropylidene-d-galactose $(-5.3 \mathrm{kcal} / \mathrm{mol})$ docked on VEGFA (PDB ID: 3V2A) had the highest affinity; however, the affinity score was invalid (>-6.0 kcal/mol) [29]. The CBMicro_013618 docked on TNFRSF1A (PDB ID: INCF) had the greatest affinity. Moreover, its score was better than Enamin_004209 $(-5.3 \mathrm{kcal} / \mathrm{mol})$ as a positive control. 
The berberine $(-6.6 \mathrm{kcal} / \mathrm{mol})$ as a positive control had a higher affinity than the stearic acid $(-4.5 \mathrm{kcal} / \mathrm{mol})$ docked on PLA2G4A (PDB ID: 1BCI). The monoolein $(-6.7 \mathrm{kcal} / \mathrm{mol})$ had the greatest affinity on PRKCA (PDB ID: 3IW4). Furthermore, its affinity score was greater than sphingosine $(-5.5 \mathrm{kcal} / \mathrm{mol})$ as a positive control. The detailed docking information is listed in Table 6.

Table 6. Binding energy of ligands and positive controls on MAPK signaling pathway.

\begin{tabular}{|c|c|c|c|c|c|c|c|}
\hline \multirow[b]{2}{*}{ Protein } & \multirow[b]{2}{*}{ Ligand } & \multirow[b]{2}{*}{$\begin{array}{l}\text { PubChem } \\
\text { ID }\end{array}$} & \multirow[b]{2}{*}{$\begin{array}{l}\text { Binding } \\
\text { Energy } \\
\text { (kcal/mol) }\end{array}$} & \multicolumn{2}{|c|}{ Grid Box } & \multirow{2}{*}{$\begin{array}{c}\begin{array}{c}\text { Hydrogen } \\
\text { Bond } \\
\text { Interactions }\end{array} \\
\begin{array}{c}\text { Amino Acid } \\
\text { Residue }\end{array} \\
\end{array}$} & \multirow{2}{*}{$\begin{array}{c}\begin{array}{c}\text { Hydrophobic } \\
\text { Interactions }\end{array} \\
\begin{array}{c}\text { Amino Acid } \\
\text { Residue }\end{array}\end{array}$} \\
\hline & & & & Center & Dimension & & \\
\hline \multirow[t]{6}{*}{$\begin{array}{l}\text { FGF1 } \\
\text { (PDB } \\
\text { ID:3OJ2) }\end{array}$} & $(\star)$ Campesterol & 173183 & -8.4 & x 9.051 & $x 40$ & $\begin{array}{l}\text { Asp283, } \\
\text { Ser252 }\end{array}$ & Arg251, Phe172, Ile257 \\
\hline & & & & у 22.527 & y 40 & & Ser220, Leu258, Ile204 \\
\hline & & & & $z-0.061$ & z 40 & & $\begin{array}{c}\text { Ala260, Gln259, } \\
\text { Tyr281 }\end{array}$ \\
\hline & $\begin{array}{l}\text { 3,4-O- } \\
\text { Isopropylidene-d- } \\
\text { galactose }\end{array}$ & 54504880 & -6.1 & x 9.051 & $x 40$ & $\begin{array}{l}\text { Arg255, } \\
\text { Thr174, } \\
\text { Phe172 }\end{array}$ & $\begin{array}{c}\text { Asn350, Asn173, } \\
\text { Ala349 }\end{array}$ \\
\hline & & & & y 22.527 & y 40 & $\begin{array}{l}\text { Asn107, } \\
\text { Gln348 }\end{array}$ & \\
\hline & & & & $z-0.061$ & z 40 & & \\
\hline \multirow{2}{*}{$\begin{array}{l}\text { Positive } \\
\text { control }\end{array}$} & (a) Suramin sodium & 8514 & -19.1 & x 9.051 & $x 40$ & Ser282, Lys27 & Arg203, Ile204, Ala260 \\
\hline & & & & $\begin{array}{l}\text { y } 22.527 \\
\mathrm{z}-0.061\end{array}$ & $\begin{array}{l}\text { y } 40 \\
\text { z } 40\end{array}$ & & $\begin{array}{c}\text { Gln259, Leu258, } \\
\text { Tyr281 } \\
\text { Asn22, Tyr23, Asp283 } \\
\text { Val249, Pro149, } \\
\text { Glu250 } \\
\text { His254, Ser252, Ile257 } \\
\text { Phe172, Val222, Ser220 }\end{array}$ \\
\hline \multirow[t]{7}{*}{$\begin{array}{l}\text { FGF2 (PDB } \\
\text { ID:1IIL) }\end{array}$} & $\begin{array}{c}(\star) \\
\text { 26,27-Dinorergosta- } \\
\text { 5,23-dien-3 } \beta \text {-ol }\end{array}$ & 22213488 & -8.0 & x 26.785 & $x 40$ & $\begin{array}{l}\text { Thr139, } \\
\text { Ser137 }\end{array}$ & $\begin{array}{l}\text { Glu323, Ser122, } \\
\text { Trp123 }\end{array}$ \\
\hline & & & & $\begin{array}{l}\mathrm{y} 14.360 \\
\mathrm{z}-1.182\end{array}$ & $\begin{array}{l}\text { y } 40 \\
\text { z } 40\end{array}$ & & $\begin{array}{c}\text { Lys313, Leu312, Ile329 } \\
\text { Leu327, Tyr328 }\end{array}$ \\
\hline & Campesterol & 173183 & -7.9 & x 26.785 & $x 40$ & Ser137 & $\begin{array}{c}\text { Thr139, Glu323, } \\
\text { Lys313 }\end{array}$ \\
\hline & & & & y 14.360 & y 40 & & Asp336, Tyr328, Ile329 \\
\hline & & & & $\mathrm{z}-1.182$ & z 40 & & \\
\hline & $\begin{array}{l}\text { Stigmasta-5,22- } \\
\text { dien-3-ol }\end{array}$ & 53870683 & -7.8 & x 26.785 & $x 40$ & $\begin{array}{l}\text { Tyr340, } \\
\text { Asp336 }\end{array}$ & Ile329, Leu312, Ser122 \\
\hline & & & & y 14.360 & y 40 & & Trp123, Ser137, Thr319 \\
\hline \multirow{10}{*}{$\begin{array}{l}\text { Positive } \\
\text { control }\end{array}$} & & & & $z-1.182$ & z 40 & & $\begin{array}{c}\text { Glu323, Asn318, } \\
\text { Lys313 } \\
\text { Leu327 }\end{array}$ \\
\hline & $\begin{array}{l}\text { 3,4-O- } \\
\text { Isopropylidene-d- } \\
\text { galactose }\end{array}$ & 54504880 & -5.6 & x 26.785 & $x 40$ & $\begin{array}{l}\text { Glu323, } \\
\text { Trp123, } \\
\text { Ser137 }\end{array}$ & Ser122 \\
\hline & & & & y 14.360 & y 40 & $\begin{array}{l}\text { Thr139, } \\
\text { Lys313 }\end{array}$ & \\
\hline & & & & $z-1.182$ & z 40 & & \\
\hline & (b) NSC172285 & 299405 & -14.7 & x 26.785 & $x 40$ & Tyr207 & Val209, Asp99, Lys119 \\
\hline & & & & y 14.360 & y 40 & & $\begin{array}{l}\text { Lys199, Gln200, } \\
\text { Glu201 }\end{array}$ \\
\hline & & & & $\mathrm{z}-1.182$ & z 40 & & \\
\hline & (b) NSC37204 & 235612 & -9.5 & x 26.785 & x 40 & $\begin{array}{l}\text { Arg210, } \\
\text { Thr121 }\end{array}$ & $\begin{array}{l}\text { Val209, Asn265, } \\
\text { Lys119 }\end{array}$ \\
\hline & & & & у 14.360 & y 40 & $\begin{array}{l}\text { Arg118, } \\
\text { Glu201 }\end{array}$ & Asp99, Gln200, Trp356 \\
\hline & & & & $\mathrm{z}-1.182$ & z 40 & & \\
\hline
\end{tabular}


Table 6. Cont.

\begin{tabular}{|c|c|c|c|c|c|c|c|}
\hline \multirow[b]{2}{*}{ Protein } & \multirow[b]{2}{*}{ Ligand } & \multirow[b]{2}{*}{$\begin{array}{l}\text { PubChem } \\
\text { ID }\end{array}$} & \multirow[b]{2}{*}{$\begin{array}{c}\text { Binding } \\
\text { Energy } \\
\text { (kcal/mol) }\end{array}$} & \multicolumn{2}{|c|}{ Grid Box } & \multirow{2}{*}{$\begin{array}{c}\begin{array}{c}\text { Hydrogen } \\
\text { Bond } \\
\text { Interactions }\end{array} \\
\begin{array}{c}\text { Amino Acid } \\
\text { Residue }\end{array} \\
\end{array}$} & \multirow{2}{*}{$\begin{array}{c}\begin{array}{c}\text { Hydrophobic } \\
\text { Interactions }\end{array} \\
\begin{array}{c}\text { Amino Acid } \\
\text { Residue }\end{array}\end{array}$} \\
\hline & & & & Center & Dimension & & \\
\hline \multirow[t]{8}{*}{$\begin{array}{l}\text { VEGFA } \\
\text { (PDB ID: } \\
\text { 3V2A) }\end{array}$} & $\begin{array}{l}(\star) \text { 3,4-O- } \\
\text { Isopropylidene-d- }\end{array}$ & 54504880 & -5.3 & x 38.009 & $x 40$ & $\begin{array}{l}\text { Gly312, } \\
\text { Ser310 }\end{array}$ & Gly255, Glu44, Ser311 \\
\hline & & & & $\begin{array}{c}y-10.962 \\
z 12.171\end{array}$ & $\begin{array}{l}\text { y } 40 \\
\text { z } 40\end{array}$ & & $\begin{array}{c}\text { Ile256, Asp257, Lys84 } \\
\text { Pro85 }\end{array}$ \\
\hline & Glyceryl palmitate & 14900 & -5.2 & x 38.009 & $x 40$ & $\begin{array}{l}\text { Pro40, } \\
\text { Asp276 }\end{array}$ & Arg275, Phe36, Lys286 \\
\hline & & & & $\begin{array}{c}\mathrm{y}-10.962 \\
\mathrm{z} 12.171\end{array}$ & $\begin{array}{l}\text { y } 40 \\
\text { z } 40\end{array}$ & & Lys48, Asn253, Ile46 \\
\hline & Monoolein & 5283468 & -5.1 & x 38.009 & $x 40$ & $\begin{array}{l}\text { Asp276, } \\
\text { Pro40 }\end{array}$ & $\begin{array}{c}\text { Arg275, Asp34, } \\
\text { Asn253 }\end{array}$ \\
\hline & Methyl palmitate & 8181 & -4.0 & $\begin{array}{c}y-10.962 \\
z \quad 12.171 \\
x 38.009 \\
y-10.962 \\
z 12.171\end{array}$ & $\begin{array}{l}\text { y } 40 \\
\text { z } 40 \\
x 40 \\
\text { y } 40 \\
\text { z } 40\end{array}$ & $\mathrm{n} / \mathrm{a}$ & $\begin{array}{c}\text { Lys48, Phe47, Ile46 } \\
\text { Phe36, Lys286 } \\
\text { Pro40, Arg275, Phe36 } \\
\text { Ile46, Asn253, Lys286 } \\
\text { Asp276 }\end{array}$ \\
\hline & $\begin{array}{l}\text { Isopropyl } \\
\text { hexanoate }\end{array}$ & 16832 & -3.9 & x 38.009 & $x 40$ & $\mathrm{n} / \mathrm{a}$ & Pro85, Ser310, Gly312 \\
\hline & & & & $\begin{array}{c}y-10.962 \\
z 12.171\end{array}$ & $\begin{array}{l}\text { y } 40 \\
\mathrm{z} 40\end{array}$ & & $\begin{array}{l}\text { Glu44, Ser311, Gly255 } \\
\text { Gln87, Lys84, Asp257 }\end{array}$ \\
\hline \multirow{3}{*}{$\begin{array}{l}\text { Positive } \\
\text { control }\end{array}$} & (c) BAW2881 & 16004702 & -7.6 & x 38.009 & $x 40$ & $\mathrm{n} / \mathrm{a}$ & Lys286, Asp34, Ser50 \\
\hline & & & & $y-10.962$ & y 40 & & Asp276, Pro40, Phe36 \\
\hline & & & & z 12.171 & $\mathrm{z} 40$ & & Ile46 \\
\hline \multirow[t]{4}{*}{$\begin{array}{l}\text { TNFRSF1A } \\
\text { (PDB ID: } \\
\text { 1NCF) }\end{array}$} & $\begin{array}{c}(\star) \\
\text { CBMicro_013618 }\end{array}$ & 1109374 & -6.8 & $x 21.259$ & $x 40$ & $\begin{array}{l}\text { Lys132, } \\
\text { Gln133 }\end{array}$ & $\begin{array}{l}\text { Glu109, Tyr106, } \\
\text { Gln130 }\end{array}$ \\
\hline & & & & $\begin{array}{l}\text { y } 14.648 \\
\text { z } 34.77\end{array}$ & $\begin{array}{l}\text { y } 40 \\
\text { z } 40\end{array}$ & & $G \ln 133$ \\
\hline & $\begin{array}{l}\text { 2-Propenoic acid, } \\
\text { 3-phenyl-, methyl } \\
\text { ester }\end{array}$ & 7644 & -5.0 & x 21.259 & $x 40$ & Lys35 & Ala62, Glu64, His34 \\
\hline & & & & $\begin{array}{l}\text { y } 14.648 \\
\text { z } 34.77\end{array}$ & $\begin{array}{l}\text { y } 40 \\
\text { z } 40\end{array}$ & & Lys35, Glu64 \\
\hline \multirow{2}{*}{$\begin{array}{l}\text { Positive } \\
\text { control }\end{array}$} & (d) Enamine_004209 & 2340496 & -5.3 & $x 21.259$ & $x 40$ & $\begin{array}{l}\text { Glu109, } \\
\text { Cvs96. Tvr106 }\end{array}$ & Asn110, Ph112, Val95 \\
\hline & & & & $\begin{array}{l}\text { y } 14.648 \\
\text { z } 34.77\end{array}$ & $\begin{array}{l}\text { y } 40 \\
\text { z } 40\end{array}$ & & $\begin{array}{l}\text { Gln82, Ser74, Thr94 } \\
\text { Arg77, Arg132 }\end{array}$ \\
\hline \multirow[t]{5}{*}{$\begin{array}{l}\text { PLA2G4A } \\
\text { (PDB ID: } \\
\text { 1BCI) }\end{array}$} & $(\star)$ Stearic acid & 5281 & -4.5 & $x-0.058$ & $x 40$ & Gly33, Lys32 & Pro42, Val30, Ile67 \\
\hline & & & & $\begin{array}{l}\text { y } 0.077 \\
\text { z } 0.285\end{array}$ & $\begin{array}{l}\text { y } 40 \\
\text { z } 40\end{array}$ & & Val127, Thr31, Gln126 \\
\hline & Methyl palmitate & 8181 & -3.9 & $\begin{array}{l}x-0.058 \\
y 0.077 \\
z 0.285\end{array}$ & $\begin{array}{l}x 40 \\
\text { y } 40 \\
\text { z } 40\end{array}$ & Lys58 & $\begin{array}{l}\text { Phe77, Pro54, Thr53 } \\
\text { Leu79, Tyr16, Glu76 } \\
\text { Ile78 }\end{array}$ \\
\hline & Palmitic acid & 985 & -3.8 & $\begin{array}{c}\mathrm{x}-0.058 \\
\mathrm{y} 0.077 \\
\mathrm{z} 0.285\end{array}$ & $\begin{array}{l}\mathrm{x} 40 \\
\mathrm{y} 40 \\
\mathrm{z} 40\end{array}$ & Thr53 & $\begin{array}{l}\text { Leu79, Phe77, Ile78 } \\
\text { Tyr16, Glu76, Pro54 }\end{array}$ \\
\hline & Myristic acid & 11005 & -3.3 & $\begin{array}{l}x-0.058 \\
y=077 \\
\text { z } 0.285\end{array}$ & $\begin{array}{l}x 40 \\
\text { y } 40 \\
\text { z } 40\end{array}$ & $\mathrm{n} / \mathrm{a}$ & $\begin{array}{l}\text { Asp55, Pro54, Tyr16 } \\
\text { Phe77, Ile78, Thr53 }\end{array}$ \\
\hline \multirow{2}{*}{$\begin{array}{l}\text { Positive } \\
\text { control }\end{array}$} & (e) Berberine & 2353 & -6.6 & $x-0.058$ & $x 40$ & $\mathrm{n} / \mathrm{a}$ & Arg59, Asp99, Asn95 \\
\hline & & & & $\begin{array}{l}\text { y } 0.077 \\
\text { z } 0.285\end{array}$ & $\begin{array}{l}\text { y } 40 \\
\mathrm{z} 40\end{array}$ & & $\begin{array}{l}\text { His62, Phe63, Asn64 } \\
\text { Arg61, Ala94, Tyr45 }\end{array}$ \\
\hline \multirow[t]{4}{*}{$\begin{array}{l}\text { PRKCA } \\
\text { (PDB ID: } \\
\text { 3IW4) }\end{array}$} & $(\star)$ Monoolein & 5283468 & -6.7 & $x-14.059$ & $x 40$ & $\begin{array}{l}\text { Asn660, } \\
\text { Leu393, } \\
\text { Asp395 }\end{array}$ & $\begin{array}{l}\text { Pro398, Lys478, } \\
\text { Glu418 }\end{array}$ \\
\hline & & & & y 38.224 & y 40 & Lys396 & $\begin{array}{c}\text { Pro666, Tyr419, } \\
\text { Arg608 }\end{array}$ \\
\hline & & & & z 32.319 & z 40 & & Val664, Gln402 \\
\hline & Glyceryl palmitate & 14900 & -6.6 & $x-14.059$ & x 40 & $\begin{array}{l}\text { Asp395, } \\
\text { Lys396, } \\
\text { Leu393 }\end{array}$ & $\begin{array}{l}\text { Leu394, Gln402, } \\
\text { Lys478 }\end{array}$ \\
\hline
\end{tabular}


Table 6. Cont

\begin{tabular}{|c|c|c|c|c|c|c|c|}
\hline \multirow[b]{2}{*}{ Protein } & \multirow[b]{2}{*}{ Ligand } & \multirow[b]{2}{*}{$\begin{array}{l}\text { PubChem } \\
\text { ID }\end{array}$} & \multirow[b]{2}{*}{$\begin{array}{c}\text { Binding } \\
\text { Energy } \\
\text { (kcal/mol) }\end{array}$} & \multicolumn{2}{|c|}{ Grid Box } & \multirow{2}{*}{$\begin{array}{c}\begin{array}{c}\text { Hydrogen } \\
\text { Bond } \\
\text { Interactions }\end{array} \\
\begin{array}{c}\text { Amino Acid } \\
\text { Residue }\end{array}\end{array}$} & \multirow{2}{*}{$\begin{array}{c}\begin{array}{c}\text { Hydrophobic } \\
\text { Interactions }\end{array} \\
\begin{array}{c}\text { Amino Acid } \\
\text { Residue }\end{array}\end{array}$} \\
\hline & & & & Center & Dimension & & \\
\hline \multirow{4}{*}{\multicolumn{2}{|c|}{ Stearic acid }} & \multirow{4}{*}{5281} & \multirow{4}{*}{-6.3} & $\begin{array}{l}\text { y } 38.224 \\
\text { z } 32.319\end{array}$ & $\begin{array}{l}\text { y } 40 \\
\text { z } 40\end{array}$ & \multirow{3}{*}{$\begin{array}{l}\text { Asn660 } \\
\text { Lys396, } \\
\text { Leu393 }\end{array}$} & \multirow{7}{*}{$\begin{array}{c}\text { Arg608, Pro666, Ile667 } \\
\text { Val664, Pro398, Pro397 } \\
\text { Pro397, Pro398, } \\
\text { Lys478 } \\
\text { Arg608, Ile667, Pro666 } \\
\text { His665, Val664, } \\
\text { Gln402 } \\
\text { Asn660, Leu394 } \\
\text { Asn660, Pro397, } \\
\text { Pro398 } \\
\text { Lys478, Pro666, } \\
\text { Arg608 } \\
\text { Glu418, Val664, } \\
\text { Gln402 } \\
\text { Leu394 }\end{array}$} \\
\hline & & & & $x-14.059$ & $x 40$ & & \\
\hline & & & & у 38.224 & y 40 & & \\
\hline & & & & z 32.319 & z 40 & & \\
\hline \multirow{3}{*}{\multicolumn{2}{|c|}{ Nonadecanoic acid }} & \multirow[t]{3}{*}{12591} & \multirow[t]{3}{*}{-6.2} & $x-14.059$ & $x 40$ & \multirow[t]{3}{*}{$\begin{array}{l}\text { Leu393, } \\
\text { Lys396 }\end{array}$} & \\
\hline & & & & y 38.224 & y 40 & & \\
\hline & & & & z 32.319 & z 40 & & \\
\hline & \multirow[t]{3}{*}{$\begin{array}{c}\text { 1,6,10-Dodecatrien- } \\
\text { 3-ol, } \\
\text { 3,7,11-trimethyl- }\end{array}$} & \multirow[t]{3}{*}{8888} & \multirow[t]{3}{*}{-6.2} & $x-14.059$ & $x 40$ & \multirow[t]{3}{*}{$\begin{array}{l}\text { Lys372, } \\
\text { Gln408, } \\
\text { Gln650 }\end{array}$} & $\begin{array}{l}\text { Val410, Thr409, } \\
\text { Gly540 }\end{array}$ \\
\hline & & & & y 38.224 & y 40 & & $\begin{array}{l}\text { Ile645, Asp539, } \\
\text { Asp503 }\end{array}$ \\
\hline & & & & z 32.319 & z 40 & & Phe538, Glu543 \\
\hline & \multirow[t]{3}{*}{$\begin{array}{l}\text { 2,5-Furandione, } \\
\text { 3-dodecenyl- }\end{array}$} & \multirow[t]{3}{*}{5362708} & \multirow[t]{3}{*}{-6.1} & $x-14.059$ & $x 40$ & \multirow[t]{3}{*}{$\begin{array}{l}\text { Lys396, } \\
\text { Asp395 }\end{array}$} & $\begin{array}{l}\text { Asn660, Gln402, } \\
\text { Pro397 }\end{array}$ \\
\hline & & & & y 38.224 & y 40 & & $\begin{array}{c}\text { Pro398, Glu552, } \\
\text { Gln662 }\end{array}$ \\
\hline & & & & z 32.319 & z 40 & & Val664, Leu394 \\
\hline & \multirow[t]{3}{*}{$\begin{array}{c}\text { 2,6-Dimethyl-3,5,7- } \\
\text { octatriene-2-ol, } \\
\text { Z,Z- }\end{array}$} & \multirow[t]{3}{*}{5363692} & \multirow[t]{3}{*}{-5.3} & $x-14.059$ & $x 40$ & \multirow[t]{3}{*}{ Gly540 } & Val410, Ile645, Asp503 \\
\hline & & & & y 38.224 & y 40 & & $\begin{array}{c}\text { Pro502, Glu543, } \\
\text { Gln650 }\end{array}$ \\
\hline & & & & z 32.319 & z 40 & & Leu546, Asp542 \\
\hline & 2-Dodecenoic acid & 5282729 & -5.1 & $x-14.059$ & $x 40$ & $\begin{array}{l}\text { Lys396, } \\
\text { Asn660, } \\
\text { Leu393 }\end{array}$ & $\begin{array}{l}\text { Leu394, Gln662, } \\
\text { Glu552 }\end{array}$ \\
\hline & & & & $\begin{array}{l}\text { y } 38.224 \\
\text { z } 32.319\end{array}$ & $\begin{array}{l}\text { y } 40 \\
\mathrm{z} 40\end{array}$ & & Val664, Gln402 \\
\hline & $\begin{array}{l}\text { 9-Octadecenoic } \\
\text { acid }\end{array}$ & 965 & -5.0 & $x-14.059$ & $x 40$ & $\begin{array}{l}\text { Leu393, } \\
\text { Lys396 }\end{array}$ & $\begin{array}{l}\text { Asn660, Glu552, } \\
\text { Gln548 }\end{array}$ \\
\hline & & & & y 38.224 & y 40 & & $\begin{array}{l}\text { His553, Ser549, } \\
\text { Gln662 }\end{array}$ \\
\hline & & & & z 32.319 & z 40 & & $\begin{array}{l}\text { Val664, Gln402, } \\
\text { Leu394 }\end{array}$ \\
\hline & Octanoic acid & 379 & -5.0 & $x-14.059$ & $x 40$ & $\begin{array}{l}\text { Asn660, } \\
\text { Lys396, } \\
\text { Gln402 }\end{array}$ & $\begin{array}{l}\text { Pro397, Lys478, } \\
\text { Pro398 }\end{array}$ \\
\hline & & & & у 38.224 & y 40 & & $\begin{array}{l}\text { Val664, Glu552, } \\
\text { Arg608 }\end{array}$ \\
\hline & & & & z 32.319 & z 40 & & \\
\hline & Methyl palmitate & 8181 & -5.0 & $x-14.059$ & $x 40$ & $\mathrm{n} / \mathrm{a}$ & $\begin{array}{l}\text { Gln377, Asn647, } \\
\text { Asp373 }\end{array}$ \\
\hline & & & & у 38.224 & y 40 & & $\begin{array}{l}\text { Ile648, Asp649, } \\
\text { Asn468 }\end{array}$ \\
\hline & & & & z 32.319 & z 40 & & $\begin{array}{c}\text { Lys465, Phe350, } \\
\text { Asp467 } \\
\text { Ile376 }\end{array}$ \\
\hline & Palmitic acid & 985 & -5.0 & $x-14.059$ & $x 40$ & $\begin{array}{l}\text { Leu393, } \\
\text { Asp395, } \\
\text { Lys396 }\end{array}$ & Pro397, Pro398, Val664 \\
\hline & & & & y 38.224 & y 40 & & $\begin{array}{l}\text { Glu552, His553, } \\
\text { Ser549 }\end{array}$ \\
\hline & & & & z 32.319 & z 40 & & \\
\hline & (E)-4-Undecenal & 5283357 & -4.8 & $x-14.059$ & $x 40$ & $\mathrm{n} / \mathrm{a}$ & $\begin{array}{l}\text { Leu394, Asn660 } \\
\text { Gln642, Pro536, Ile645 }\end{array}$ \\
\hline & & & & y 38.224 & y 40 & & $\begin{array}{l}\text { Gly540, Val410, } \\
\text { Gln650 }\end{array}$ \\
\hline
\end{tabular}


Table 6. Cont

\begin{tabular}{|c|c|c|c|c|c|c|c|}
\hline \multirow[b]{2}{*}{ Protein } & \multirow[b]{2}{*}{ Ligand } & \multirow[b]{2}{*}{$\begin{array}{l}\text { PubChem } \\
\text { ID }\end{array}$} & \multirow[b]{2}{*}{$\begin{array}{c}\text { Binding } \\
\text { Energy } \\
(\mathrm{kcal} / \mathrm{mol})\end{array}$} & \multicolumn{2}{|c|}{ Grid Box } & \multirow{2}{*}{\begin{tabular}{|c|}
$\begin{array}{c}\text { Hydrogen } \\
\text { Bond } \\
\text { Interactions }\end{array}$ \\
$\begin{array}{c}\text { Amino Acid } \\
\text { Residue }\end{array}$ \\
\end{tabular}} & \multirow{2}{*}{$\begin{array}{c}\begin{array}{c}\text { Hydrophobic } \\
\text { Interactions }\end{array} \\
\begin{array}{c}\text { Amino Acid } \\
\text { Residue }\end{array}\end{array}$} \\
\hline & & & & Center & Dimension & & \\
\hline & & & & z 32.319 & z 40 & & $\begin{array}{c}\text { Glu543, Asp542, } \\
\text { Asp503 } \\
\text { Leu546 }\end{array}$ \\
\hline & Myristic acid & 11005 & -4.8 & $x-14.059$ & $x 40$ & $\begin{array}{l}\text { Lys396, } \\
\text { Gln402 }\end{array}$ & $\begin{array}{l}\text { Asp395, Leu393, } \\
\text { Leu394 }\end{array}$ \\
\hline & & & & y 38.224 & y 40 & & $\begin{array}{c}\text { Pro398, Val664, } \\
\text { Gln662 }\end{array}$ \\
\hline & & & & z 32.319 & z 40 & & Asn660 \\
\hline & Nonanoic acid & 8158 & -4.7 & $x-14.059$ & $x 40$ & $\begin{array}{l}\text { Leu393, } \\
\text { Lys396 }\end{array}$ & $\begin{array}{c}\text { Leu394, Asn660, } \\
\text { Pro397 }\end{array}$ \\
\hline & & & & y 38.224 & y 40 & & $\begin{array}{c}\text { Gln402, Gln662, } \\
\text { Val664 }\end{array}$ \\
\hline & & & & z 32.319 & z 40 & & \\
\hline & Heptadec-8-ene & 520230 & -4.6 & $x-14.059$ & $x 40$ & $\mathrm{n} / \mathrm{a}$ & $\begin{array}{c}\text { Asn647, Asp424, } \\
\text { Met426 }\end{array}$ \\
\hline & & & & $\begin{array}{l}\text { y } 38.224 \\
\text { z } 32.319\end{array}$ & $\begin{array}{l}\text { y } 40 \\
\text { z } 40\end{array}$ & & $\begin{array}{c}\text { Gln377, Ile376, Phe350 } \\
\text { Asp467, Asp373 }\end{array}$ \\
\hline \multirow[t]{3}{*}{$\begin{array}{l}\text { Positive } \\
\text { control }\end{array}$} & (f) Sphingosine & 5280335 & -5.5 & $x-14.059$ & $x 40$ & $\begin{array}{l}\text { Asn660, } \\
\text { Gln662, }\end{array}$ & $\begin{array}{l}\text { Pro397, Gln402, } \\
\text { Val664 }\end{array}$ \\
\hline & & & & y 38.224 & y 40 & & $\begin{array}{l}\text { Gln548, Glu552, } \\
\text { His553 }\end{array}$ \\
\hline & & & & z 32.319 & z 40 & & $\begin{array}{l}\text { Leu394, Ser549, } \\
\text { Asp395 }\end{array}$ \\
\hline
\end{tabular}

${ }^{(\star)}$ Compound with the greatest affinity on each target; ${ }^{(a)}$ FGF1 antagonist; ${ }^{(b)}$ FGF2 antagonist; ${ }^{(c)}$ VEGFA antagonist; ${ }^{(d)}$ TNFRSF1A antagonist; (e) PLA2G4A antagonist; ${ }^{(\mathrm{f})}$ PRKCA antagonist.
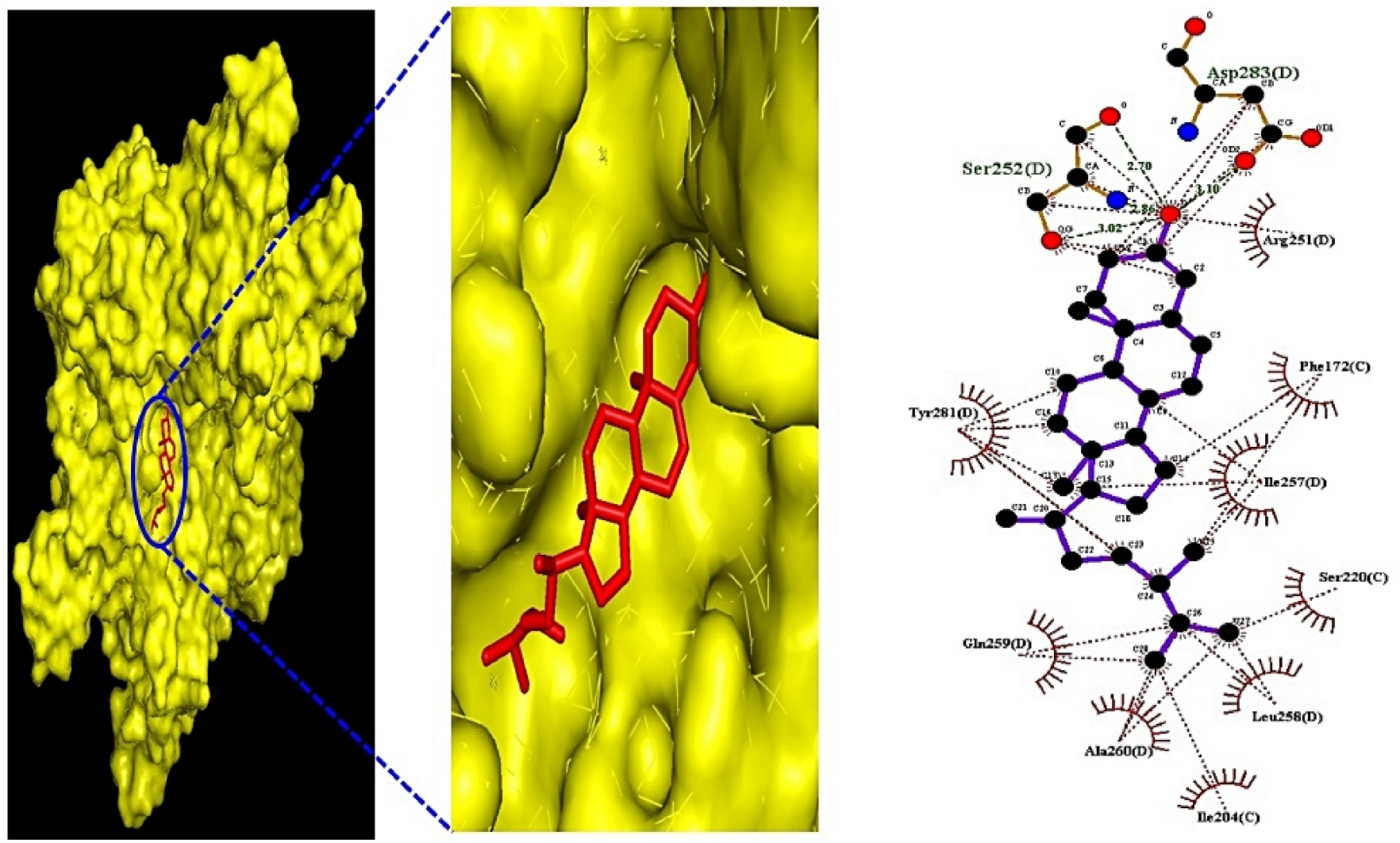

(A)

Figure 8. Cont. 

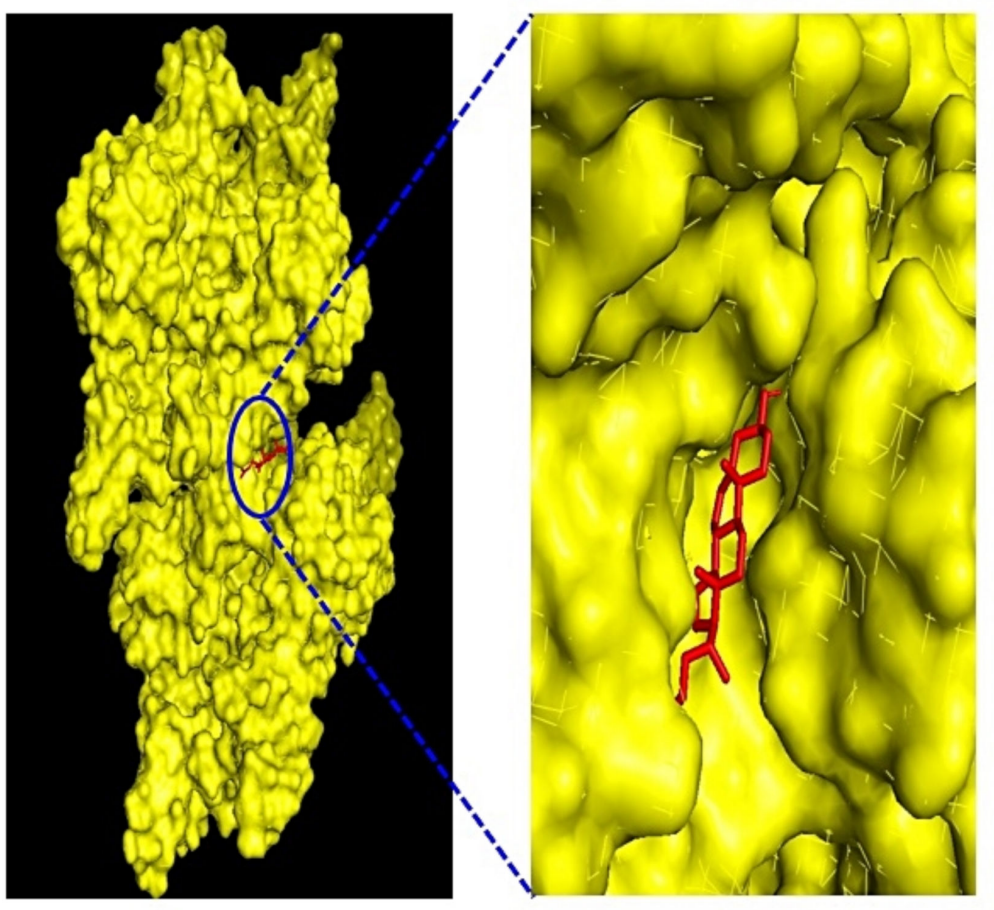

(B)
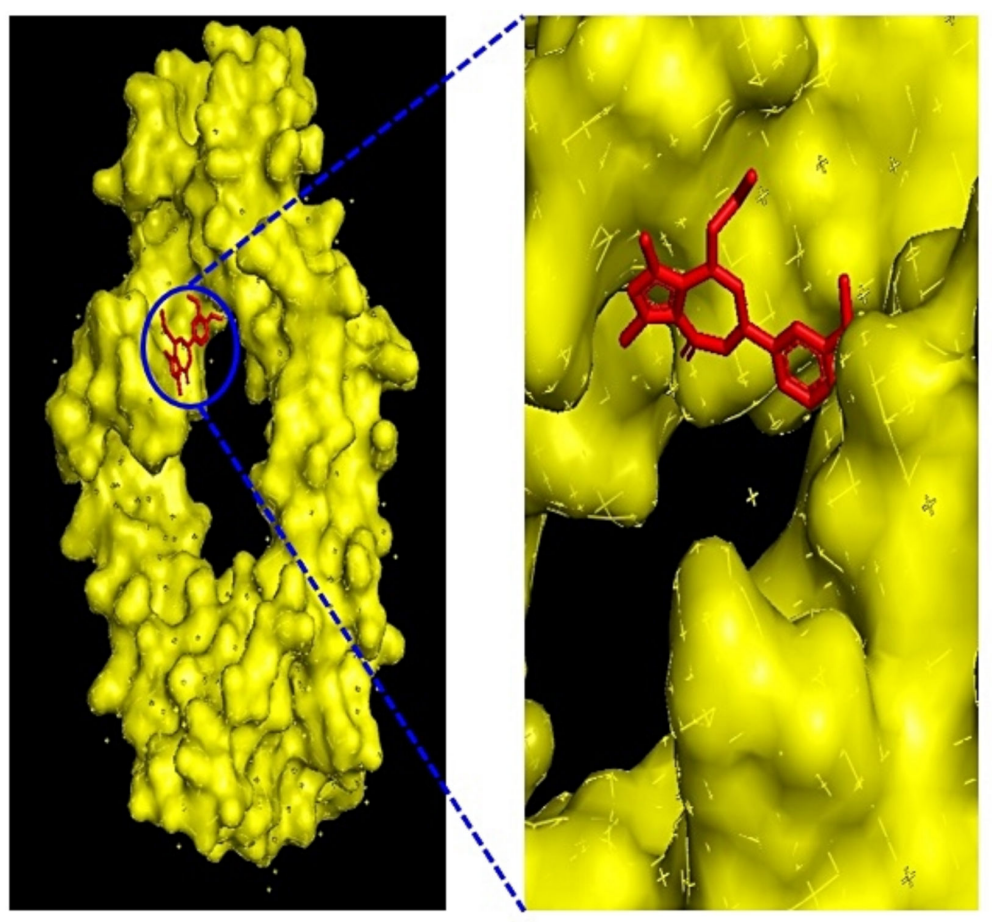

(C)
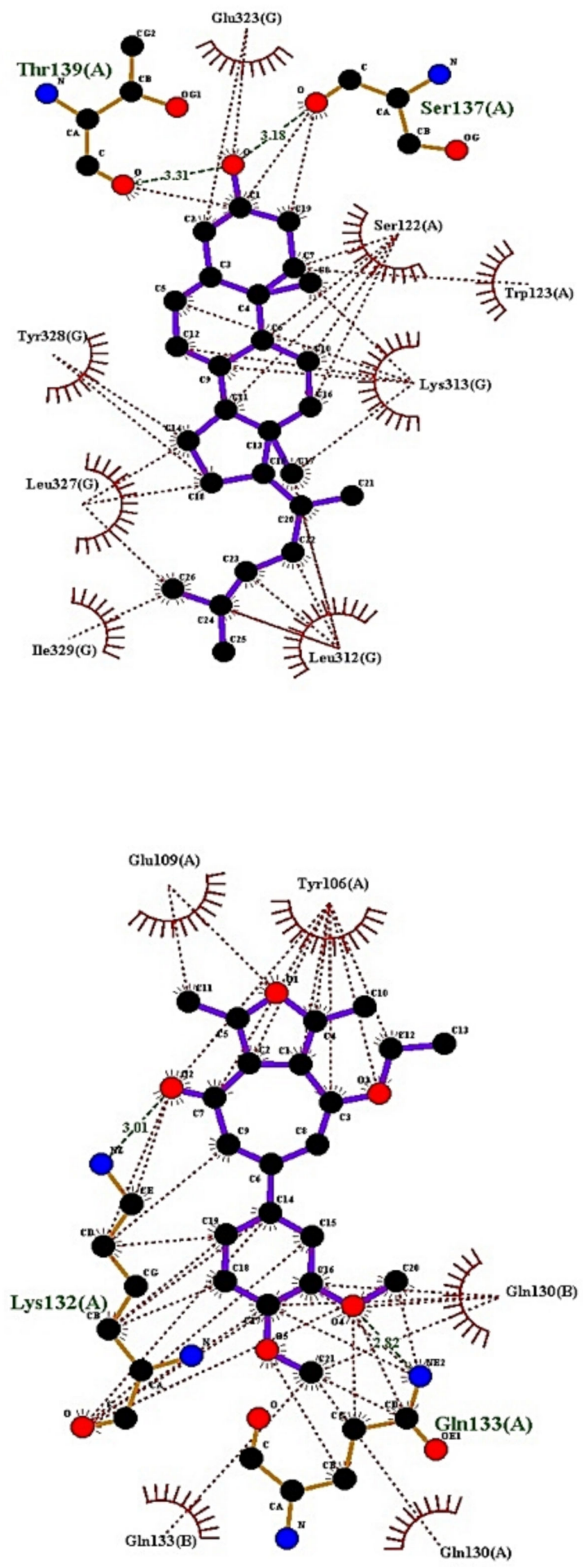

Figure 8. Cont. 

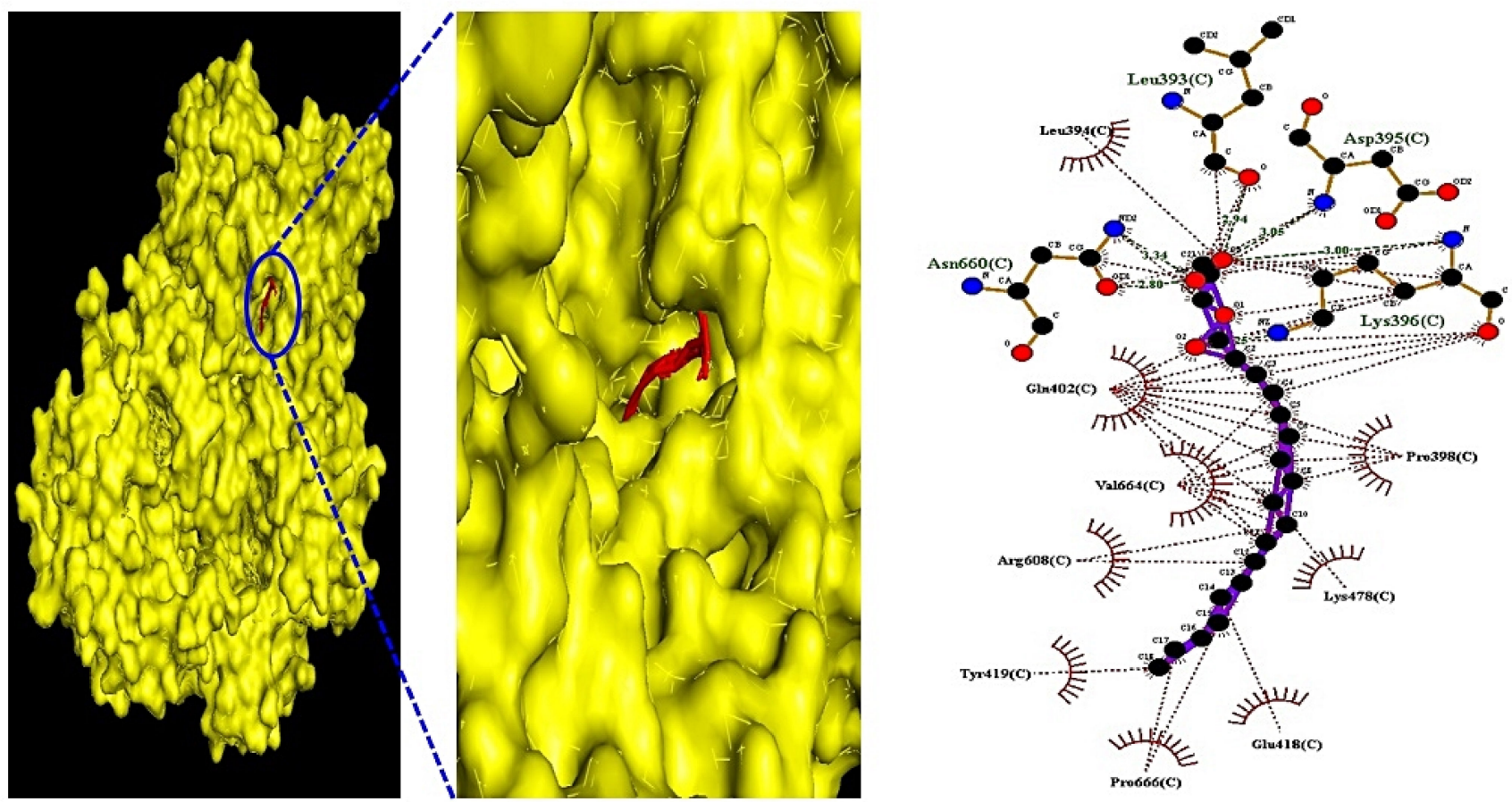

(D)

Figure 8. (A) MDT of campesterol (PubChem ID: 173183) on FGF1 (PDB ID: 3OJ2). (B) MDT of 26,27-Dinorergosta-5,23-dien3ß-ol (PubChem ID: 22213488) on FGF2 (PDB ID: 1IIL). (C) MDT of CBMicro_013618 (PubChem ID: 1109374) on TNFRSF1A (PDB ID: 1NCF). (D) MDT of monoolein (PubChem ID: 5283468) on PRKCA (PDB ID: 1NCF).

\subsection{MDT of 5 Targets and 43 Chemical Compounds Associated with PPAR Signaling Pathway}

From both SEA and STP databases, it was revealed that Peroxisome ProliferatorActivated Receptor Alpha (PPARA) is related to 32 chemical compounds, Peroxisome Proliferator-Activated Receptor Delta (PPARD) to 18 chemical compounds, Peroxisome Proliferator-Activated Receptor Gamma (PPARG) to 17 chemical compounds, FABP3 to 26 chemical compounds, and MMP to five chemical compounds. The MDT performed to obtain affinity between targets and ligands exhibited the highest affinity complex, as displayed in Figure 9. Noticeably, $\beta$-Caryophyllene $(-8.6 \mathrm{kcal} / \mathrm{mol})$ docked on PPARA (PDB ID: 3SP6) had the greatest affinity, which had a higher score than Clofibrate $(-6.4 \mathrm{kcal} / \mathrm{mol})$, Gemfibrozil $(-6.3 \mathrm{kcal} / \mathrm{mol})$, Ciprofibrate $(-5.4 \mathrm{kcal} / \mathrm{mol})$, Bezafibrate $(-5.8 \mathrm{kcal} / \mathrm{mol})$, and Fenofibrate $(-5.4 \mathrm{kcal} / \mathrm{mol})$ as five positive controls. Stigmasta-5,22dien-3-ol $(-8.6 \mathrm{kcal} / \mathrm{mol})$ docked on PPARD (PDB ID: 5U3Q) had the greatest affinity, which had a better score than Cardarine $(-8.5 \mathrm{kcal} / \mathrm{mol})$ as a positive control. NSC402953 $(-8.2 \mathrm{kcal} / \mathrm{mol})$ docked on PPARG (PDB ID: 3E00) had the highest affinity, which had better than Pioglitazone $(-7.7 \mathrm{kcal} / \mathrm{mol})$, Rosiglitazone $(-7.4 \mathrm{kcal} / \mathrm{mol})$, and Lobeglitazone $(-7.3 \mathrm{kcal} / \mathrm{mol})$ as three positive controls. Monoolein $(-8.9 \mathrm{kcal} / \mathrm{mol})$ docked on FABP3 (PDB ID: 5HZ9) had the greatest affinity; specifically, there was no positive control on FABP3 (PDB ID: 5HZ9). 2-Propenoic acid, 3-phenyl-, methyl ester $(-5.0 \mathrm{kcal} / \mathrm{mol})$ docked on MMP1 (PDB ID: 1SU3) had the highest affinity, which had lower affinity than Batimastat $(-6.7 \mathrm{kcal} / \mathrm{mol})$, and Ilomastat $(-6.5 \mathrm{kcal} / \mathrm{mol})$ as two positive controls. The detailed docking information is listed in Table 7.

3.10. Identification of the Uppermost Seven Targets and Eight Compounds from Two Key Signaling Pathways against $R A$

Campesterol on FGF1, 26,27-Dinorergosta-5,23-dien-3ß-ol on FGF2, CBMicro_013618 on TNFRSF1A, and monoolein on PRKCA on MAPK signaling pathway had significant 
valid affinity score to develop new promising ligands. Additionally, $\beta$-Caryophyllene on PPARA, Stigmasta-5,22-dien-3-ol on PPARD, NSC0402953 on PPARG, and monoolein on FABP3 had an important valid score on the PPAR signaling pathway.
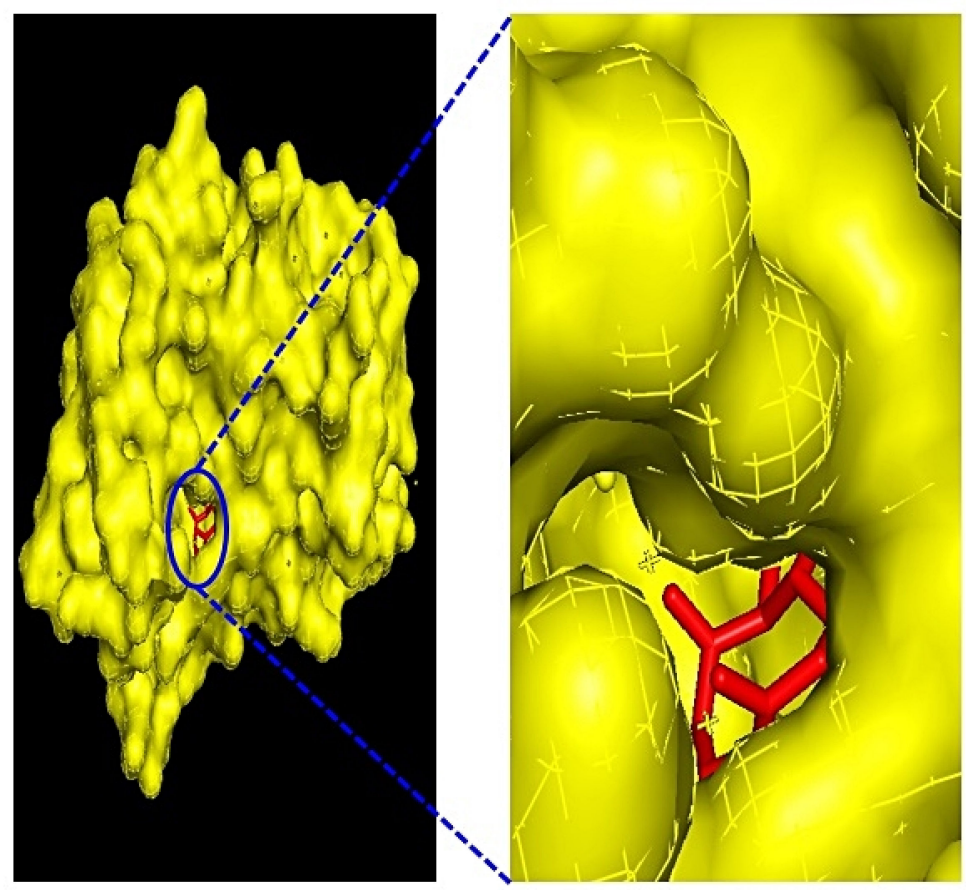

(A)
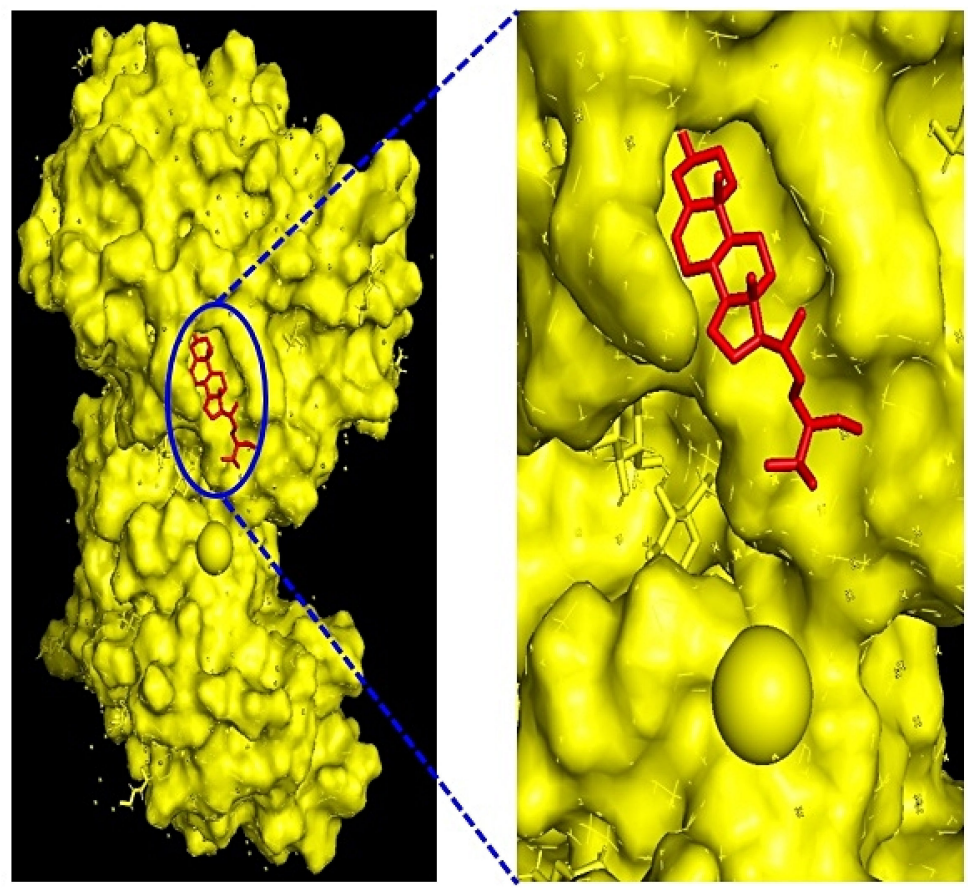

(B)
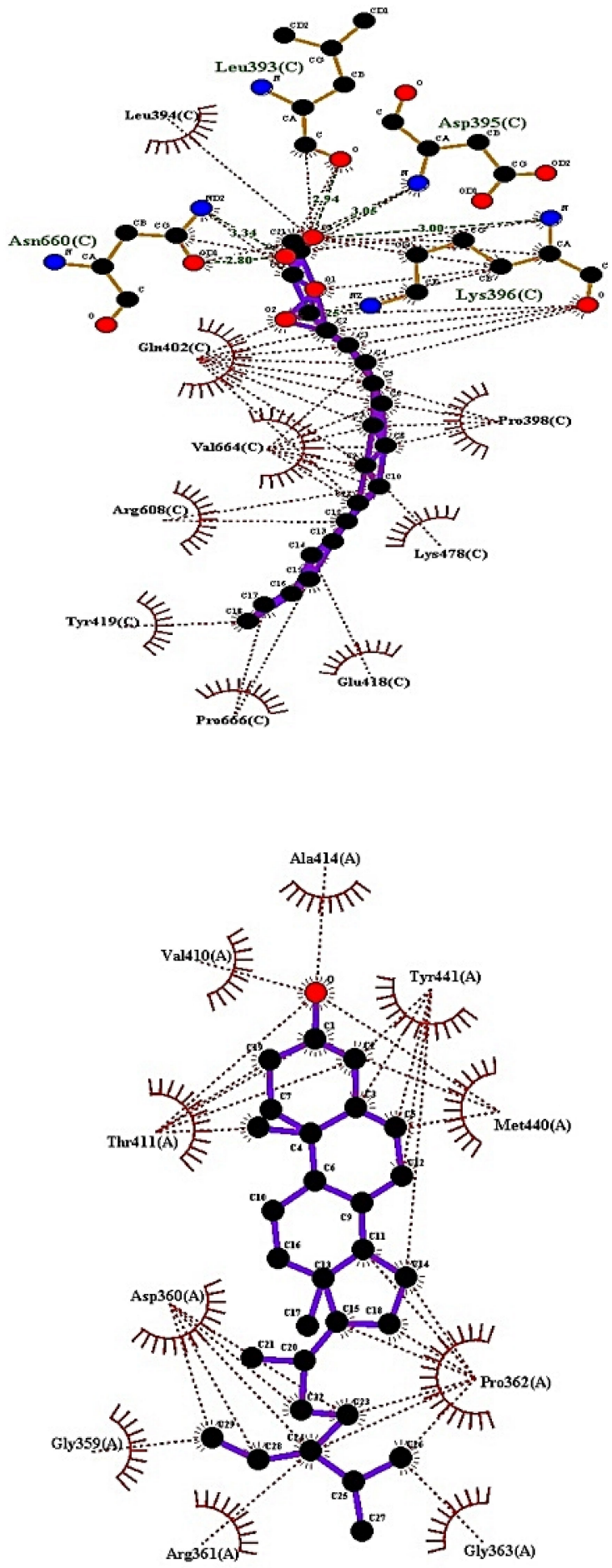

Figure 9. Cont. 

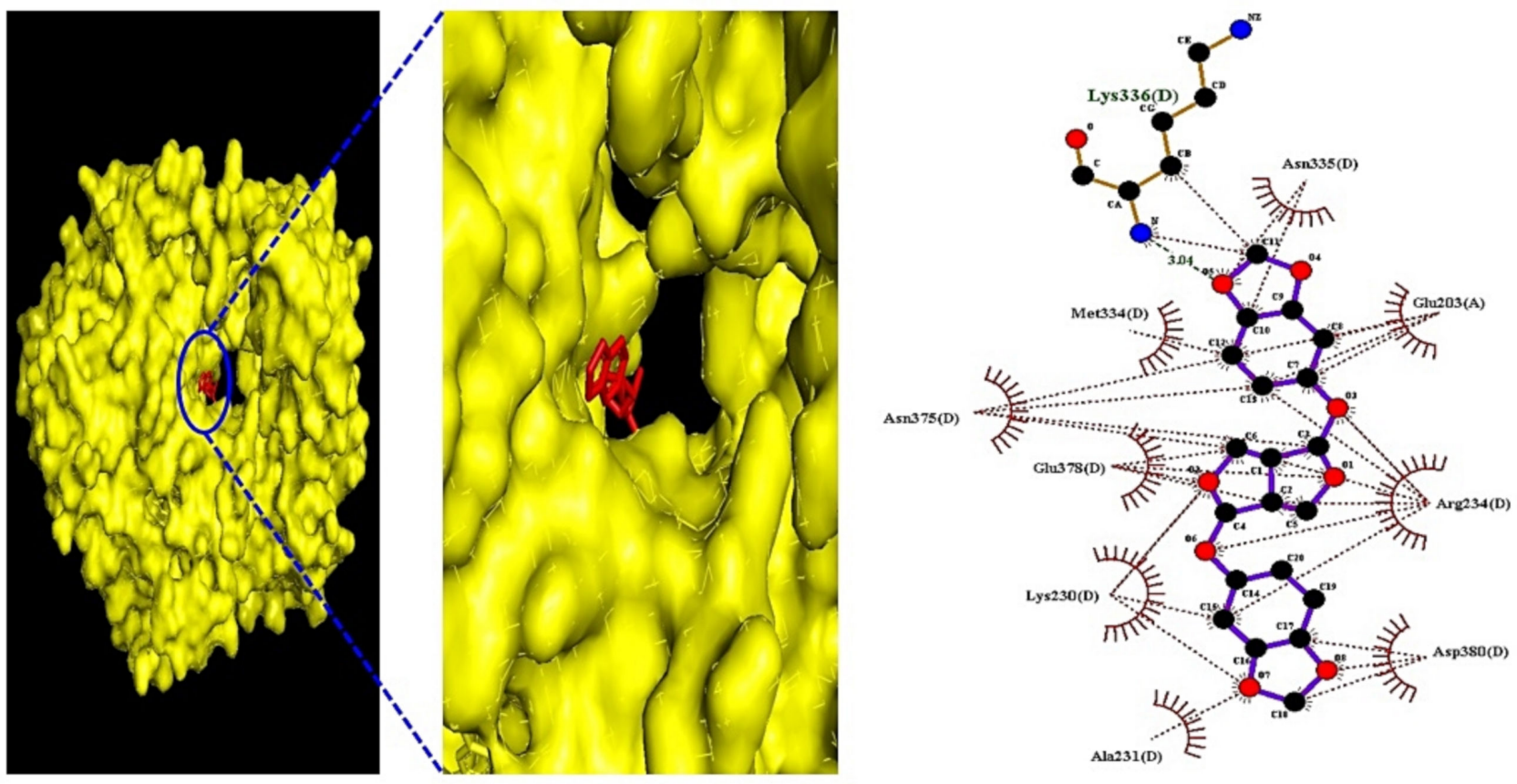

(C)
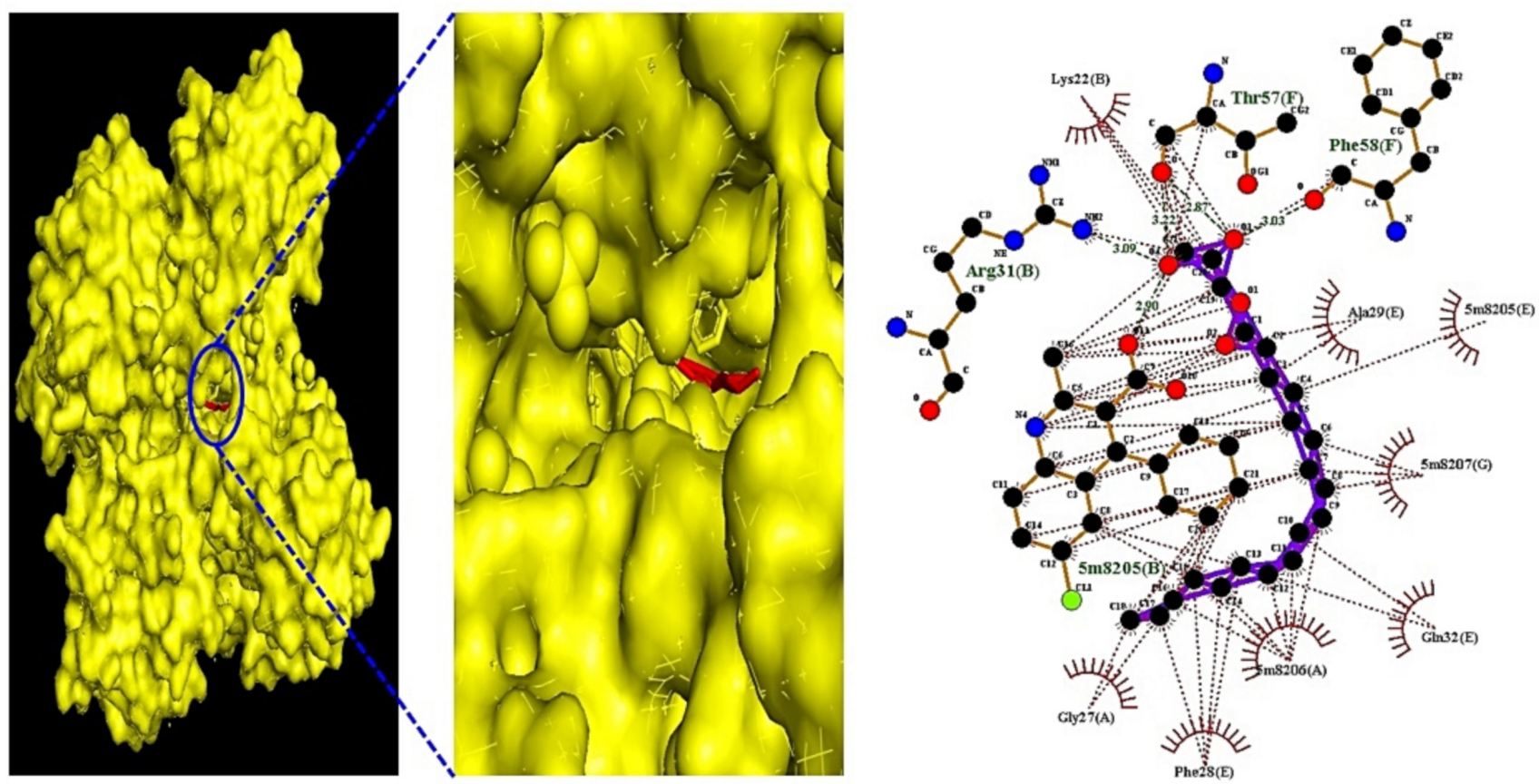

(D)

Figure 9. (A) MDT of $\beta$-Caryophyllene (PubChem ID: 5281515) on PPARA (PDB ID: 3SP6). (B) MDT of Stigmasta-5,22-dien3-ol (PubChem ID: 53870683) on PPARD (PDB ID: 5U3Q). (C) MDT of NSC402953 (PubChem ID:345349) on PPARG (PDB ID: 3E00). (D) MDT of monoolein (PubChem ID: 5283468) on FABP3 (PDB ID: 5HZ9).

\subsection{Toxicological Properties of 8 Compounds}

Additionally, toxicological properties of Campesterol; 26,27-Dinorergosta-5,23-dien3ß-ol; CBMicro_013618; monoolein; $\beta$-Caryophyllene; Stigmasta-5,22-dien-3-ol; and NSC0402953 were predicted by admetSAR online tool. Our result indicated that chemical compounds did not disclose Ames toxicity, carcinogenic properties, acute oral toxicity, and rat acute toxicity properties (Table 8 ). 
Table 7. Binding energy of ligands and positive controls on PPAR signaling pathway.

\begin{tabular}{|c|c|c|c|c|c|c|c|}
\hline \multirow[b]{2}{*}{ Protein } & \multirow[b]{2}{*}{ Ligand } & \multirow[b]{2}{*}{$\begin{array}{l}\text { PubChem } \\
\text { ID }\end{array}$} & \multirow[b]{2}{*}{$\begin{array}{c}\text { Binding } \\
\text { Energy } \\
\text { (kcal/mol) }\end{array}$} & \multicolumn{2}{|c|}{ Grid Box } & \multirow{2}{*}{$\begin{array}{c}\begin{array}{c}\text { Hydrogen } \\
\text { Bond } \\
\text { Interactions }\end{array} \\
\text { Amino } \\
\text { Acid } \\
\text { Residue }\end{array}$} & \multirow{2}{*}{$\begin{array}{c}\begin{array}{c}\text { Hydrophobic } \\
\text { Interactions }\end{array} \\
\begin{array}{c}\text { Amino Acid } \\
\text { Residue }\end{array}\end{array}$} \\
\hline & & & & Center & Dimension & & \\
\hline \multirow[t]{27}{*}{$\begin{array}{l}\text { PPARA } \\
\text { (PDB ID: } \\
\text { 3SP6) }\end{array}$} & $(\star) \beta$-Caryophyllene & 5281515 & -8.6 & x 8.006 & x 40 & $\mathrm{n} / \mathrm{a}$ & Leu321, Leu331, Gly335 \\
\hline & & & & $\begin{array}{l}y-0.459 \\
z 23.392\end{array}$ & $\begin{array}{l}\text { y } 40 \\
\mathrm{z} 40\end{array}$ & & $\begin{array}{l}\text { Val324, Met220, Tyr334 } \\
\text { Ala333, Thr279, Asn219 } \\
\text { Thr283 }\end{array}$ \\
\hline & Cadina-1(10),4-diene & 10223 & -7.4 & $\begin{array}{c}x 8.006 \\
y-0.459 \\
z 23.392\end{array}$ & $\begin{array}{l}x 40 \\
\text { y } 40 \\
\text { z } 40\end{array}$ & $\mathrm{n} / \mathrm{a}$ & $\begin{array}{c}\text { Met220, Leu331, Val324 } \\
\text { Thr279, Thr283, Leu321 } \\
\text { Ile317, Met320 }\end{array}$ \\
\hline & $\begin{array}{l}\text { 26,27-Dinorergosta- } \\
\text { 5,23-dien-3beta-ol }\end{array}$ & 22213488 & -7.0 & x 8.006 & $x 40$ & Lys345 & Glu356, Asp353, Pro357 \\
\hline & & & & $\begin{array}{l}\mathrm{y}-0.459 \\
\mathrm{z} 23.392\end{array}$ & $\begin{array}{l}\text { y } 40 \\
\text { z } 40\end{array}$ & & $\begin{array}{l}\text { Leu443, His440, Glu439 } \\
\text { Leu436, Lys358, Asp360 }\end{array}$ \\
\hline & Clionasterol & 457801 & -6.7 & $\begin{array}{c}x 8.006 \\
y-0.459 \\
z \quad 23.392\end{array}$ & $\begin{array}{l}\mathrm{x} 40 \\
\mathrm{y} 40 \\
\mathrm{z} 40\end{array}$ & Lys345 & $\begin{array}{l}\text { Asp360, Pro357, Glu439 } \\
\text { His440, Leu443, Asp353 } \\
\text { Glu356 }\end{array}$ \\
\hline & $\begin{array}{c}\text { Cyclohexene, } \\
\text { 4-(4-ethylcyclohexyl)- } \\
\text { 1-pentyl- }\end{array}$ & 543386 & -6.6 & x 8.006 & $x 40$ & $\mathrm{n} / \mathrm{a}$ & $\begin{array}{l}\text { Met320, Phe218, } \\
\text { Met220 }\end{array}$ \\
\hline & & & & $\begin{array}{l}y-0.459 \\
z 23.392\end{array}$ & $\begin{array}{l}\text { y } 40 \\
\mathrm{z} 40\end{array}$ & & $\begin{array}{l}\text { Thr279, Val332, Ala333 } \\
\text { Tyr334, Thr283, Asn219 }\end{array}$ \\
\hline & $\begin{array}{l}\text { Spiro[4.4]nona-1,3- } \\
\text { diene, } \\
\text { 1,2-dimethyl- }\end{array}$ & 570800 & -6.5 & x 8.006 & $x 40$ & $\mathrm{n} / \mathrm{a}$ & Leu321, Leu331, Val324 \\
\hline & & & & $\begin{array}{l}y-0.459 \\
z 23.392\end{array}$ & $\begin{array}{l}\text { y } 40 \\
\mathrm{z} 40\end{array}$ & & $\begin{array}{c}\text { Met320, Asn219, Thr283 } \\
\text { Thr279, Met220 }\end{array}$ \\
\hline & $\begin{array}{l}\text { Methyldecahydrona- } \\
\text { phthalene }\end{array}$ & 34193 & -6.4 & x 8.006 & $x 40$ & $\mathrm{n} / \mathrm{a}$ & Met320, Val324, Met220 \\
\hline & & & & $\begin{array}{l}y-0.459 \\
z 23.392\end{array}$ & $\begin{array}{l}\text { y } 40 \\
\text { z } 40\end{array}$ & & $\begin{array}{c}\text { Asn219, Thr279, Thr283 } \\
\text { Leu321 }\end{array}$ \\
\hline & $\begin{array}{c}1,3,4,5- \\
\text { Tetrahydroxycycloh- } \\
\text { exanecarboxylic } \\
\text { acid }\end{array}$ & 1064 & -6.3 & x 8.006 & $x 40$ & $\begin{array}{l}\text { Ile317, } \\
\text { Glu286, } \\
\text { Asn219 }\end{array}$ & $\begin{array}{c}\text { Met320, Met220, } \\
\text { Leu321 }\end{array}$ \\
\hline & & & & $\begin{array}{l}y-0.459 \\
z 23.392\end{array}$ & $\begin{array}{l}\text { y } 40 \\
\text { z } 40\end{array}$ & Thr283 & \\
\hline & $\begin{array}{c}\text { Stigmasta-5,22-dien- } \\
\text { 3-ol }\end{array}$ & 53870683 & -6.3 & x 8.006 & $x 40$ & $\mathrm{n} / \mathrm{a}$ & Arg465, Glu462, Ser688 \\
\hline & & & & $\begin{array}{l}y-0.459 \\
z 23.392\end{array}$ & $\begin{array}{l}\text { y } 40 \\
\mathrm{z} 40\end{array}$ & & $\begin{array}{c}\text { Val306, Asn303, Thr307 } \\
\text { Tyr311, Gly390, Pro389 } \\
\text { Lys310, Asp466 }\end{array}$ \\
\hline & Terpinolene & 11463 & -6.2 & x 8.006 & $x 40$ & $\mathrm{n} / \mathrm{a}$ & Thr279, Tyr334, Val324 \\
\hline & & & & $y-0.459$ & y 40 & & $\begin{array}{c}\text { Met220, Met320, } \\
\text { Leu321 }\end{array}$ \\
\hline & $\beta$-Phellandrene & 11142 & -6.0 & $\begin{array}{c}\text { z } 23.392 \\
x \quad 8.006 \\
y-0.459 \\
z 23.392\end{array}$ & $\begin{array}{l}\text { z } 40 \\
x 40 \\
y 40 \\
\text { z } 40\end{array}$ & $\mathrm{n} / \mathrm{a}$ & $\begin{array}{c}\text { Thr283, Asn219 } \\
\text { Leu331, Val324, Leu321 } \\
\text { Ile317, Thr283, Met320 } \\
\text { Thr279 }\end{array}$ \\
\hline & Citronellic acid & 10402 & -5.9 & x 8.006 & $x 40$ & $\begin{array}{l}\text { Thr283, } \\
\text { Glu286, } \\
\text { Met220 }\end{array}$ & Met320, Asn219, Tyr334 \\
\hline & & & & $\mathrm{y}-0.459$ & y 40 & & Gly335, Leu321, Val324 \\
\hline & & & & z 23.392 & z 40 & & Ile317 \\
\hline & Stearic acid & 5281 & -5.7 & x 8.006 & $x 40$ & $\mathrm{n} / \mathrm{a}$ & $\begin{array}{c}\text { Phe361, Asp432, } \\
\text { Leu436 }\end{array}$ \\
\hline & & & & $\begin{array}{l}y-0.459 \\
z 23.392\end{array}$ & $\begin{array}{l}\text { y } 40 \\
\text { z } 40\end{array}$ & & $\begin{array}{c}\text { Glu433, His440, Leu443 } \\
\text { Asp353, Gln442, Ile446 } \\
\text { Pro357, Lys358 }\end{array}$ \\
\hline & Monoolein & 5283468 & -5.7 & x 8.006 & $x 40$ & $\begin{array}{l}\text { Asn261, } \\
\text { Lys257 }\end{array}$ & Leu258, His274, Cys275 \\
\hline & & & & $\begin{array}{l}y-0.459 \\
z 23.392\end{array}$ & $\begin{array}{l}\text { y } 40 \\
\mathrm{z} 40\end{array}$ & & Ala333, Val255, Cys278 \\
\hline & $\begin{array}{l}\text { 2,6-Octadiene, } \\
\text { 2,6-dimethyl- }\end{array}$ & 5365898 & -5.7 & x 8.006 & $x 40$ & $\mathrm{n} / \mathrm{a}$ & Met320, Phe218, Leu331 \\
\hline
\end{tabular}


Table 7. Cont.

\begin{tabular}{|c|c|c|c|c|c|c|c|}
\hline \multirow[b]{2}{*}{ Protein } & \multirow[b]{2}{*}{ Ligand } & \multirow[b]{2}{*}{$\begin{array}{l}\text { PubChem } \\
\text { ID }\end{array}$} & \multirow[b]{2}{*}{$\begin{array}{c}\text { Binding } \\
\text { Energy } \\
\text { (kcal/mol) }\end{array}$} & \multicolumn{2}{|c|}{ Grid Box } & \multirow{2}{*}{\begin{tabular}{|c|}
$\begin{array}{c}\text { Hydrogen } \\
\text { Bond } \\
\text { Interactions }\end{array}$ \\
Amino \\
Acid \\
Residue
\end{tabular}} & \multirow{2}{*}{$\begin{array}{c}\begin{array}{c}\text { Hydrophobic } \\
\text { Interactions }\end{array} \\
\begin{array}{c}\text { Amino Acid } \\
\text { Residue }\end{array}\end{array}$} \\
\hline & & & & Center & Dimension & & \\
\hline & \multirow{3}{*}{ Citronellol } & \multirow[b]{2}{*}{8842} & \multirow[b]{2}{*}{-5.5} & $\begin{array}{l}y-0.459 \\
z 23.392\end{array}$ & $\begin{array}{l}\text { y } 40 \\
\mathrm{z} 40\end{array}$ & \multirow{3}{*}{ Thr283 } & Val324, Met220, Leu321 \\
\hline & & & & $\begin{array}{c}x 8.006 \\
y-0.459\end{array}$ & & & $\begin{array}{l}\text { Leu331, Val324, Ile317 } \\
\text { Leu321, Met320, Thr279 }\end{array}$ \\
\hline & & \multirow{2}{*}{31253} & \multirow{2}{*}{-5.5} & $\begin{array}{l}\text { z } 23.392 \\
\times 8.006\end{array}$ & $\begin{array}{l}\text { z } 40 \\
\times 40\end{array}$ & & Val332, Val324, Ile317 \\
\hline & \multirow{3}{*}{ (E)- $\beta$-Ocimene } & & & $y-0.459$ & y 40 & $\mathrm{n} / \mathrm{a}$ & Leu321, Met220, Thr283 \\
\hline & & \multirow{2}{*}{5281553} & \multirow{2}{*}{-5.5} & $\begin{array}{l}\text { z } 23.392 \\
\times 8.006\end{array}$ & $\begin{array}{l}\text { z } 40 \\
\times 40\end{array}$ & \multirow[t]{2}{*}{$\mathrm{n} / \mathrm{a}$} & $\begin{array}{c}\text { Met320, Leu331 } \\
\text { Thr283, Ile317, Met320 }\end{array}$ \\
\hline & & & & $\begin{array}{l}y-0.459 \\
z 23.392\end{array}$ & $\begin{array}{l}\text { y } 40 \\
\mathrm{z} 40\end{array}$ & & $\begin{array}{c}\text { Tyr334, Val332, Val324 } \\
\text { Gly335, Leu331, Thr279 } \\
\text { Leu321 }\end{array}$ \\
\hline & \multirow[t]{2}{*}{ Citronellal } & \multirow[t]{2}{*}{7794} & \multirow[t]{2}{*}{-5.2} & x 8.006 & $x 40$ & \multirow[t]{2}{*}{ Thr283 } & $\begin{array}{c}\text { Leu321, Met220, } \\
\text { Met320 }\end{array}$ \\
\hline & & & & $\begin{array}{l}y-0.459 \\
z 23.392\end{array}$ & $\begin{array}{l}\text { y } 40 \\
\mathrm{z} 40\end{array}$ & & $\begin{array}{l}\text { Val324, Asn219, Thr279 } \\
\text { Ile317 }\end{array}$ \\
\hline & \multirow{2}{*}{ Heptadec-8-ene } & \multirow{2}{*}{520230} & \multirow{2}{*}{-5.2} & x 8.006 & $x 40$ & $\mathrm{n} / \mathrm{a}$ & Leu321, Ile317, Thr283 \\
\hline & & & & $\begin{array}{l}y-0.459 \\
z 23.392\end{array}$ & $\begin{array}{l}\text { y } 40 \\
\text { z } 40\end{array}$ & & $\begin{array}{c}\text { Thr279, Val255, Ala333 } \\
\text { Tyr334, Leu331, Val332 } \\
\text { Val324 }\end{array}$ \\
\hline & Myristic acid & 11005 & -5.2 & x 8.006 & $x 40$ & $\begin{array}{c}\text { Tyr334, } \\
\text { Ala333,Thr279 }\end{array}$ & Val332, Met220, Met320 \\
\hline & & & & $\mathrm{y}-0.459$ & y 40 & & Ile317, Leu321, Thr283 \\
\hline & Nonanoic acid & 8158 & -5.1 & $\begin{array}{c}\text { z } 23.392 \\
x \quad 8.006 \\
y-0.459 \\
z 23.392\end{array}$ & $\begin{array}{l}\text { z } 40 \\
x 40 \\
\text { y } 40 \\
\text { z } 40\end{array}$ & Ala333 & $\begin{array}{c}\text { Asn219 } \\
\text { Leu331, Leu321, Val332 } \\
\text { Ile317, Thr283, Met320 } \\
\text { Val324, Thr279 }\end{array}$ \\
\hline & $\begin{array}{c}\text { 10-Bromoundecanoic } \\
\text { acid }\end{array}$ & 543401 & -5.1 & x 8.006 & $x 40$ & $\mathrm{n} / \mathrm{a}$ & Met320, Val324, Leu321 \\
\hline & & & & $\begin{array}{l}y-0.459 \\
z 23.392\end{array}$ & $\begin{array}{l}\text { y } 40 \\
\text { z } 40\end{array}$ & & $\begin{array}{l}\text { Thr279, Leu331, Val332 } \\
\text { Asn219, Tyr334, Met220 }\end{array}$ \\
\hline & $\begin{array}{l}\text { 2-Methyl-Z,Z-3,13- } \\
\text { octadecadienol }\end{array}$ & 5364412 & -5.0 & x 8.006 & $x 40$ & $\mathrm{n} / \mathrm{a}$ & Leu254, Ala333, Cys275 \\
\hline & & & & $\begin{array}{l}y-0.459 \\
z 23.392\end{array}$ & $\begin{array}{l}\text { y } 40 \\
\mathrm{z} 40\end{array}$ & & $\begin{array}{c}\text { Tyr334, Ile317, Met320 } \\
\text { Thr283, Leu321, Leu331 } \\
\text { Val324, Ala250, Thr279 } \\
\text { Ile241, Val255 }\end{array}$ \\
\hline & Octanoic acid & 379 & -5.0 & x 8.006 & $x 40$ & $\begin{array}{l}\text { Asn219, } \\
\text { Thr283, } \\
\text { Met220 }\end{array}$ & Phe218, Leu321, Val324 \\
\hline & & & & $\begin{array}{l}y-0.459 \\
z 23.392\end{array}$ & $\begin{array}{l}\text { y } 40 \\
\text { z } 40\end{array}$ & Glu286 & Leu331, Met320 \\
\hline & Nonadecanoic acid & 12591 & -4.9 & $\begin{array}{c}x 8.006 \\
y-0.459 \\
z 23.392\end{array}$ & $\begin{array}{l}x 40 \\
\text { y } 40 \\
\text { z } 40\end{array}$ & $\mathrm{n} / \mathrm{a}$ & $\begin{array}{c}\text { Asn336, Leu254, Ala333 } \\
\text { Ala250, Cys275, Val255 } \\
\text { Tyr334 }\end{array}$ \\
\hline & $\begin{array}{c}3- \\
\text { Hydroxycyclohexanone }\end{array}$ & 439950 & -4.9 & x 8.006 & $x 40$ & Met220 & $\begin{array}{l}\text { Met320, Phe218, } \\
\text { Asn219 }\end{array}$ \\
\hline & & & & $\begin{array}{l}y-0.459 \\
z 23.392\end{array}$ & $\begin{array}{l}\text { y } 40 \\
\mathrm{z} 40\end{array}$ & & Glu286 \\
\hline & Palmitic acid & 985 & -4.9 & $\times 8.006$ & $\times 40$ & $\mathrm{n} / \mathrm{a}$ & Glu251, Val332, Ile241 \\
\hline & & & & $\begin{array}{l}y-0.459 \\
z 23.392\end{array}$ & $\begin{array}{l}\text { y } 40 \\
\text { z } 40\end{array}$ & & $\begin{array}{c}\text { Ala333, Thr279, Val255 } \\
\text { Tyr334, Leu258, Cys275 } \\
\text { Ala250, Leu254 }\end{array}$ \\
\hline & 3-Methylcyclohexene & 11573 & -4.6 & x 8.006 & $\times 40$ & $\mathrm{n} / \mathrm{a}$ & Met320, Ile317, Leu321 \\
\hline & & & & $\begin{array}{l}y-0.459 \\
z 23.392\end{array}$ & $\begin{array}{l}\text { y } 40 \\
\text { z } 40\end{array}$ & & Thr283 \\
\hline & 9-Octadecenoic acid & 965 & -4.4 & $\times 8.006$ & $\times 40$ & $\mathrm{n} / \mathrm{a}$ & Glu251, Ala250, Leu254 \\
\hline & & & & $y-0.459$ & y 40 & & Val255, Ile241, Ala333 \\
\hline & & & & z 23.392 & z 40 & & Asn336, Tyr334, Cys275 \\
\hline & 2-Tetradecynoic acid & 324386 & -4.1 & x 8.006 & x 40 & Thr307 & Glu462, Ser688, Gln691 \\
\hline & & & & $\begin{array}{l}y-0.459 \\
z 23.392\end{array}$ & $\begin{array}{l}\text { y } 40 \\
\mathrm{z} 40\end{array}$ & & $\begin{array}{l}\text { Tyr311, Lys310, Asn303 } \\
\text { Val306 }\end{array}$ \\
\hline & Methyl palmitate & 8181 & -3.7 & x 8.006 & $x 40$ & $\mathrm{n} / \mathrm{a}$ & Tyr311, Gln691, Pro389 \\
\hline & & & & $\begin{array}{l}y-0.459 \\
z 23.392\end{array}$ & $\begin{array}{l}\text { y } 40 \\
\text { z } 40\end{array}$ & & $\begin{array}{l}\text { Lys310, Thr307, Asn303 } \\
\text { Val306, Ser688, Glu462 }\end{array}$ \\
\hline $\begin{array}{l}\text { Positive } \\
\text { control }\end{array}$ & (a) Clofibrate & 2796 & -6.4 & x 8.006 & $x 40$ & Thr283 & Ala333, Tyr334, Asn219 \\
\hline
\end{tabular}


Table 7. Cont.

\begin{tabular}{|c|c|c|c|c|c|c|c|}
\hline \multirow[b]{2}{*}{ Protein } & \multirow[b]{2}{*}{ Ligand } & \multirow[b]{2}{*}{$\begin{array}{l}\text { PubChem } \\
\text { ID }\end{array}$} & \multirow[b]{2}{*}{$\begin{array}{c}\text { Binding } \\
\text { Energy } \\
\text { (kcal/mol) }\end{array}$} & \multicolumn{2}{|c|}{ Grid Box } & \multirow{2}{*}{\begin{tabular}{|c|}
$\begin{array}{c}\text { Hydrogen } \\
\text { Bond } \\
\text { Interactions }\end{array}$ \\
Amino \\
Acid \\
Residue
\end{tabular}} & \multirow{2}{*}{$\begin{array}{c}\begin{array}{c}\text { Hydrophobic } \\
\text { Interactions }\end{array} \\
\begin{array}{c}\text { Amino Acid } \\
\text { Residue }\end{array}\end{array}$} \\
\hline & & & & Center & Dimension & & \\
\hline & & & & $y-0.459$ & y 40 & & $\begin{array}{l}\text { Met320, Leu321, } \\
\text { Met220 }\end{array}$ \\
\hline & & & & z 23.392 & z 40 & & $\begin{array}{c}\text { Phe218, Val332, Val324 } \\
\text { Thr279 }\end{array}$ \\
\hline & (a) Gemfibrozil & 3463 & -6.3 & x 8.006 & $x 40$ & Tyr468 & Tyr464, Lys448, Leu456 \\
\hline & & & & $\begin{array}{l}y-0.459 \\
z 23.392\end{array}$ & $\begin{array}{l}\text { y } 40 \\
\text { z } 40\end{array}$ & & Arg465, Gln442, Ala441 \\
\hline & (a) Ciprofibrate & 2763 & -5.4 & x 8.006 & $x 40$ & $\begin{array}{l}\text { Ala333, } \\
\text { Thr279 }\end{array}$ & Lys257, Cys278, Tyr334 \\
\hline & & & & $\begin{array}{l}y-0.459 \\
z 23.392\end{array}$ & $\begin{array}{l}\text { y } 40 \\
\text { z } 40\end{array}$ & & Cys275, Val255, Leu258 \\
\hline & (a) Bezafibrate & 39042 & -5.8 & x 8.006 & $x 40$ & $\begin{array}{l}\text { Thr307, } \\
\text { Ser688 }\end{array}$ & Asn303, Glu462, Val306 \\
\hline & & & & $\begin{array}{l}y-0.459 \\
z 23.392\end{array}$ & $\begin{array}{l}\text { y } 40 \\
\text { z } 40\end{array}$ & & Leu690, Lys310, Gly390 \\
\hline & (a) Fenofibrate & 3339 & -5.4 & $\times 8.006$ & $\times 40$ & $\mathrm{n} / \mathrm{a}$ & Gln435, Ala431, Asp360 \\
\hline & & & & $\begin{array}{l}y-0.459 \\
z 23.392\end{array}$ & $\begin{array}{l}\text { y } 40 \\
\text { z } 40\end{array}$ & & $\begin{array}{l}\text { Pro357, Leu436, Glu439 } \\
\text { Lys364, Phe361, Asp432 }\end{array}$ \\
\hline \multirow{24}{*}{$\begin{array}{l}\text { PPARD } \\
\text { (PDB } \\
\text { ID:5U3Q) }\end{array}$} & 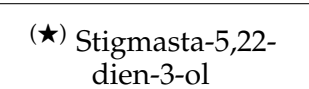 & 53870683 & -8.6 & x 39.265 & $x 40$ & $\mathrm{n} / \mathrm{a}$ & Ala414, Tyr441, Met440 \\
\hline & & & & $\begin{array}{l}y-18.736 \\
z 119.392\end{array}$ & $\begin{array}{l}\text { y } 40 \\
\mathrm{z} 40\end{array}$ & & $\begin{array}{l}\text { Pro362, Gly363, Arg361 } \\
\text { Gly359, Asp360, Thr411 } \\
\text { Val410 }\end{array}$ \\
\hline & Clionasterol & 457801 & -7.3 & $\begin{array}{c}x 39.265 \\
y-18.736 \\
z 119.392\end{array}$ & $\begin{array}{l}\text { x } 40 \\
\text { y } 40 \\
\text { z } 40\end{array}$ & Met440 & $\begin{array}{c}\text { Ala414, Tyr441, Tyr284 } \\
\text { Pro362, Arg361, Asp360 } \\
\text { Val410, Thr411 }\end{array}$ \\
\hline & $\begin{array}{l}\text { 26,27-Dinorergosta- } \\
5,23 \text {-dien-3beta-ol }\end{array}$ & 22213488 & -7.3 & x 39.265 & $x 40$ & $\mathrm{n} / \mathrm{a}$ & Lys188, Glu262, Lys265 \\
\hline & & & & $\begin{array}{l}y-18.736 \\
z \\
119.392\end{array}$ & $\begin{array}{l}\text { y } 40 \\
\text { z } 40\end{array}$ & & Ser266, Ser271 \\
\hline & Stearic acid & 5281 & -6.8 & & $\begin{array}{l}x 40 \\
\text { y } 40\end{array}$ & $\mathrm{n} / \mathrm{a}$ & $\begin{array}{l}\text { Glu288, Tyr284, Asp439 } \\
\text { Asp360, Val367, Gly359 }\end{array}$ \\
\hline & & & & z 119.392 & $\mathrm{z} 40$ & & $\begin{array}{l}\text { Leu364, Gly363, Arg361 } \\
\text { Pro362, Met440, Thr411 } \\
\text { Tyr441 }\end{array}$ \\
\hline & $\begin{array}{c}1,3,4,5- \\
\text { Tetrahydroxycyclohe- } \\
\text { xanecarboxylic } \\
\text { acid }\end{array}$ & 1064 & -6.6 & x 39.265 & $x 40$ & $\begin{array}{l}\text { Tyr441, } \\
\text { Glu288 }\end{array}$ & Ala414, Thr411, Val410 \\
\hline & & & & $\begin{array}{l}y-18.736 \\
z \\
119.392\end{array}$ & $\begin{array}{l}\text { y } 40 \\
\text { z } 40\end{array}$ & & $\begin{array}{c}\text { Tyr284, } \\
\text { Met440 }\end{array}$ \\
\hline & Nonadecanoic acid & 12591 & -5.8 & $\times 39.265$ & $\times 40$ & Asp360 & Pro362, Tyr441, Met440 \\
\hline & & & & $y-18.736$ & y 40 & & Val410, Glu288, Arg407 \\
\hline & Citronellal & 7794 & -5.7 & $\begin{array}{l}\text { z } 119.392 \\
\times 39.265\end{array}$ & $\begin{array}{l}\mathrm{Z} 40 \\
\times 40\end{array}$ & $\mathrm{n} / \mathrm{a}$ & $\begin{array}{l}\text { Thr411, Arg361, Tyr284 } \\
\text { Asn307, Ala306, Thr252 }\end{array}$ \\
\hline & & & & $\mathrm{y}-18.736$ & y 40 & & Trp228, Arg248, Val305 \\
\hline & Nonanoic acid & 8158 & -5.7 & $\begin{array}{l}\text { z } 119.392 \\
\times 39.265\end{array}$ & $\begin{array}{l}\text { Z } 40 \\
\times 40\end{array}$ & Thr256 & $\begin{array}{c}\text { Gln230, Lys229 } \\
\text { Glu255 Asn307. Lys229 }\end{array}$ \\
\hline & & & & $\mathrm{y}-18.736$ & y 40 & & Trp228, Ala306, Thr252 \\
\hline & Citronellic acid & 10402 & -5.3 & $\begin{array}{l}\text { z } 119.392 \\
\times 39.265\end{array}$ & $\begin{array}{l}\text { Z } 40 \\
\times 40\end{array}$ & $\mathrm{n} / \mathrm{a}$ & $\begin{array}{c}\text { Glu259, Asn191 } \\
\text { Arg361, Tyr441, Thr411 }\end{array}$ \\
\hline & & & & $\mathrm{y}-18.736$ & y 40 & & Met440, Tyr284 \\
\hline & Myristic acid & 11005 & -5.2 & $\begin{array}{l}\text { z } 119.392 \\
\times 39.265\end{array}$ & $\begin{array}{l}\text { Z } 40 \\
\times 40\end{array}$ & Tyr441 & Ala414, Glu288, Pro362 \\
\hline & & & & $\mathrm{y}-18.736$ & y 40 & & Arg361, Asp439, Tyr284 \\
\hline & & & & z 119.392 & z 40 & & $\begin{array}{l}\text { Arg407, Thr411, Met440 } \\
\text { Val410 }\end{array}$ \\
\hline & Octanoic acid & 379 & -5.1 & x 39.265 & $x 40$ & $\begin{array}{l}\text { Lys229, } \\
\text { Gln230, }\end{array}$ & Cys251, Trp228, Val305 \\
\hline & & & & $\begin{array}{l}y-18.736 \\
z \\
119.392\end{array}$ & $\begin{array}{l}\text { y } 40 \\
\text { z } 40\end{array}$ & & Ala306, Thr252 \\
\hline & 9-Octadecenoic acid & 965 & -5.0 & $\times 39.265$ & $\times 40$ & $\mathrm{n} / \mathrm{a}$ & Met440, Thr411, Tyr441 \\
\hline & & & & $\begin{array}{l}y-18.736 \\
z \\
119.392\end{array}$ & $\begin{array}{l}\text { y } 40 \\
\text { z } 40\end{array}$ & & $\begin{array}{c}\text { Pro362, Tyr284, Arg361 } \\
\text { Val410 }\end{array}$ \\
\hline
\end{tabular}


Table 7. Cont

\begin{tabular}{|c|c|c|c|c|c|c|c|}
\hline \multirow[b]{2}{*}{ Protein } & \multirow[b]{2}{*}{ Ligand } & \multirow[b]{2}{*}{$\begin{array}{l}\text { PubChem } \\
\text { ID }\end{array}$} & \multirow[b]{2}{*}{$\begin{array}{c}\text { Binding } \\
\text { Energy } \\
\text { (kcal/mol) }\end{array}$} & \multicolumn{2}{|c|}{ Grid Box } & \multirow{2}{*}{$\begin{array}{c}\begin{array}{c}\text { Hydrogen } \\
\text { Bond } \\
\text { Interactions }\end{array} \\
\text { Amino } \\
\text { Acid } \\
\text { Residue }\end{array}$} & \multirow{2}{*}{$\begin{array}{c}\begin{array}{c}\text { Hydrophobic } \\
\text { Interactions }\end{array} \\
\begin{array}{c}\text { Amino Acid } \\
\text { Residue }\end{array}\end{array}$} \\
\hline & & & & Center & Dimension & & \\
\hline & $\begin{array}{l}\text { 3-Hydroxycyclo- } \\
\text { hexanone }\end{array}$ & 439950 & -5.0 & x 39.265 & $x 40$ & $\begin{array}{l}\text { Arg407, } \\
\text { Thr411 }\end{array}$ & Val410, Met440, Tyr441 \\
\hline & Citronellol & 8842 & -4.8 & $\begin{array}{l}y-18.736 \\
\mathrm{z} 119.392 \\
x 39.265 \\
\mathrm{y}-18.736\end{array}$ & $\begin{array}{l}\text { y } 40 \\
\text { z } 40 \\
x 40 \\
\text { y } 40\end{array}$ & Arg361 & $\begin{array}{c}\text { Asp439, Met440, Tyr441 } \\
\text { Tyr284, Val410 }\end{array}$ \\
\hline & Palmitic acid & 985 & -4.6 & $\begin{array}{l}\text { z } 119.392 \\
x 39.265 \\
y-18.736 \\
\text { z } 119.392\end{array}$ & $\begin{array}{l}\text { z } 40 \\
x 40 \\
y 40 \\
\text { z } 40\end{array}$ & $\mathrm{n} / \mathrm{a}$ & $\begin{array}{c}\text { Tyr441, Pro362, Arg361 } \\
\text { Val410, Tyr284, Glu288 } \\
\text { Met440, Thr411, Ala414 } \\
\text { Arg407 }\end{array}$ \\
\hline & $\begin{array}{c}\text { 10-Bromoundecanoic } \\
\text { acid }\end{array}$ & 543401 & -4.6 & x 39.265 & $x 40$ & $\begin{array}{l}\text { Arg361, } \\
\text { Tyr284 }\end{array}$ & Tyr441, Met440, Pro362 \\
\hline & Methyl palmitate & 8181 & -4.5 & $\begin{array}{l}y-18.736 \\
z 119.392 \\
x \quad 39.265\end{array}$ & $\begin{array}{l}\text { y } 40 \\
\text { z } 40 \\
\text { x } 40\end{array}$ & $\mathrm{n} / \mathrm{a}$ & $\begin{array}{c}\text { Thr411, Glu288 } \\
\text { Thr411, Tyr441, Pro362 }\end{array}$ \\
\hline \multirow{3}{*}{$\begin{array}{l}\text { Positive } \\
\text { control }\end{array}$} & \multirow{3}{*}{ (b) Cardarine } & \multirow{3}{*}{9803963} & \multirow{3}{*}{-8.5} & $\begin{array}{l}y-18.736 \\
z-119.392\end{array}$ & $\begin{array}{l}\text { y } 40 \\
\text { z } 40\end{array}$ & & $\begin{array}{c}\text { Met440, Arg361, Tyr284 } \\
\text { Glu288 }\end{array}$ \\
\hline & & & & x 39.265 & $x 40$ & \multirow{2}{*}{$\begin{array}{l}\text { Ser271, } \\
\text { Ser272 }\end{array}$} & Lys265, Glu262, Ser266 \\
\hline & & & & $\begin{array}{l}y-18.736 \\
z 119.392\end{array}$ & $\begin{array}{l}\text { y } 40 \\
\text { z } 40\end{array}$ & & $\begin{array}{c}\text { Lys265, Ser271, Pro268 } \\
\text { Ser269 }\end{array}$ \\
\hline \multirow[t]{18}{*}{$\begin{array}{l}\text { PPARG } \\
\text { (PDB ID: } \\
\text { 3E00) }\end{array}$} & \multirow[t]{3}{*}{$(\star)$ NSC402953 } & \multirow[t]{3}{*}{345349} & \multirow[t]{3}{*}{-8.2} & x 2.075 & $x 40$ & \multirow[t]{3}{*}{ Lys336 } & $\begin{array}{c}\text { Asn335, Glu203, } \\
\text { Arg234 }\end{array}$ \\
\hline & & & & y 31.910 & y 40 & & Asp380, Ala231, Lys230 \\
\hline & & & & z 18.503 & z 40 & & $\begin{array}{c}\text { Glu378, Asn375, } \\
\text { Met334 }\end{array}$ \\
\hline & \multirow{2}{*}{$\begin{array}{l}\text { 26,27-Dinorergosta- } \\
\text { 5,23-dien-3beta-ol }\end{array}$} & \multirow[t]{2}{*}{22213488} & \multirow[t]{2}{*}{-8.0} & x 2.075 & $x 40$ & \multirow[t]{2}{*}{ Asn375 } & Val372, Asn335, Val205 \\
\hline & & & & $\begin{array}{l}\text { y } 31.910 \\
\text { z } 18.503\end{array}$ & $\begin{array}{l}\text { y } 40 \\
\text { z } 40\end{array}$ & & Val163, Glu207, Glu208 \\
\hline & \multirow{2}{*}{$\begin{array}{l}\text { Stigmasta-5,22-dien- } \\
\text { 3-ol }\end{array}$} & \multirow[t]{2}{*}{53870683} & \multirow[t]{2}{*}{-7.8} & $x 2.075$ & $x 40$ & \multirow[t]{2}{*}{ Asn375 } & Arg202, Glu203, Lys336 \\
\hline & & & & $\begin{array}{l}\text { y } 31.910 \\
\text { z } 18.503\end{array}$ & $\begin{array}{l}\text { y } 40 \\
\mathrm{z} 40\end{array}$ & & $\begin{array}{c}\text { Val163, Arg164, Gln206 } \\
\text { Lys165, Glu208, Glu207 } \\
\text { Val372, Asn335 }\end{array}$ \\
\hline & \multirow[t]{2}{*}{ Clionasterol } & \multirow[t]{2}{*}{457801} & \multirow[t]{2}{*}{-7.7} & $\begin{array}{c}\text { x } 2.075 \\
\text { y } 31.910\end{array}$ & $\begin{array}{l}x 40 \\
\text { y } 40\end{array}$ & \multirow[t]{2}{*}{ Asn375 } & $\begin{array}{l}\text { Asn335, Val372, Lys336 } \\
\text { Val163, Arg164, Glu208 }\end{array}$ \\
\hline & & & & z 18.503 & z 40 & & $\begin{array}{c}\text { Asp166, Glu207, } \\
\text { Arg202 }\end{array}$ \\
\hline & \multirow[t]{2}{*}{ Terpinyl propionate } & \multirow[t]{2}{*}{62328} & -6.6 & $x 2.075$ & $x 40$ & Glu343 & Leu340, Ile341, Leu333 \\
\hline & & & & y 31.910 & y 40 & & $\begin{array}{l}\text { Leu228, Met329, } \\
\text { Arg288 }\end{array}$ \\
\hline & Nonadecanoic acid & 12591 & -5.9 & $\begin{array}{l}\text { z } 18.503 \\
\text { x } 2.075 \\
\text { y } 31.910 \\
\text { z } 18.503\end{array}$ & $\begin{array}{l}\text { z } 40 \\
x 40 \\
\text { y } 40 \\
\text { z } 40\end{array}$ & Glu351 & $\begin{array}{l}\text { Lys354, Thr168, Tyr189 } \\
\text { Tyr169, Thr162, Leu167 } \\
\text { Tyr192, Arg202, Arg350 }\end{array}$ \\
\hline & & & & & & & Asp337, Lys336, Gln193 \\
\hline & Stearic acid & 5281 & -5.9 & x 2.075 & $x 40$ & $\begin{array}{l}\text { Ser342, } \\
\text { Cvs285 }\end{array}$ & Ile341, Phe226, Ile296 \\
\hline & Citronellic acid & 10402 & -5.2 & $\begin{array}{l}\text { y } 31.910 \\
\text { z } 18.503 \\
\text { x } 2.075 \\
\text { y } 31.910 \\
\text { z } 18.503\end{array}$ & $\begin{array}{l}\text { y } 40 \\
\text { z } 40 \\
x 40 \\
\text { y } 40 \\
\text { z } 40\end{array}$ & $\mathrm{n} / \mathrm{a}$ & $\begin{array}{l}\text { Glu295, Ala292, Met329 } \\
\text { Leu333, Leu228, Arg288 } \\
\text { Glu295, Arg288, Ala292 } \\
\text { Leu228, Leu333, Met329 }\end{array}$ \\
\hline & Palmitic acid & 985 & -5.1 & $\times 2.075$ & $\times 40$ & $\begin{array}{l}\text { Glu291, } \\
\text { Arg288 }\end{array}$ & Glu343, Leu333, Leu228 \\
\hline & Myristic acid & 11005 & -5.1 & $\begin{array}{l}\text { y } 31.910 \\
\text { z } 18.503 \\
x 2.075 \\
\text { y } 31.910\end{array}$ & $\begin{array}{l}\text { y } 40 \\
\text { z } 40 \\
x 40 \\
\text { y } 40\end{array}$ & Glu343 & $\begin{array}{l}\text { Met229, Ala292, Ile326 } \\
\text { Glu295 } \\
\text { Arg288, Ser342, Leu333 } \\
\text { Met329, Leu228, Glu295 }\end{array}$ \\
\hline & Nonanoic acid & 8158 & -5.0 & $\begin{array}{l}210.005 \\
\times 2.075\end{array}$ & $\begin{array}{l}240 \\
\times 40\end{array}$ & Lys354 & Arg350, Glu351, Tyr169 \\
\hline
\end{tabular}


Table 7. Cont.

\begin{tabular}{|c|c|c|c|c|c|c|c|}
\hline \multirow[b]{2}{*}{ Protein } & \multirow[b]{2}{*}{ Ligand } & \multirow[b]{2}{*}{$\begin{array}{l}\text { PubChem } \\
\text { ID }\end{array}$} & \multirow[b]{2}{*}{$\begin{array}{c}\text { Binding } \\
\text { Energy } \\
\text { (kcal/mol) }\end{array}$} & \multicolumn{2}{|c|}{ Grid Box } & \multirow{2}{*}{$\begin{array}{c}\begin{array}{c}\text { Hydrogen } \\
\text { Bond } \\
\text { Interactions }\end{array} \\
\text { Amino } \\
\text { Acid } \\
\text { Residue }\end{array}$} & \multirow{2}{*}{$\begin{array}{c}\begin{array}{c}\text { Hydrophobic } \\
\text { Interactions }\end{array} \\
\begin{array}{c}\text { Amino Acid } \\
\text { Residue }\end{array}\end{array}$} \\
\hline & & & & Center & Dimension & & \\
\hline & Citronellal & 7794 & -4.9 & $\begin{array}{l}\text { y } 31.910 \\
\text { z } 18.503 \\
\text { x } 2.075 \\
\text { y } 31.910 \\
\text { z } 18.503\end{array}$ & $\begin{array}{l}\text { y } 40 \\
\text { z } 40 \\
x 40 \\
\text { y } 40 \\
\text { z } 40\end{array}$ & Gln193 & $\begin{array}{c}\text { Tyr192, Tyr189, Leu167 } \\
\text { Gln193, Asp337, Lys336 } \\
\text { Tyr189, Arg202, Leu167 } \\
\text { Thr162, Tyr192, Asp337 } \\
\text { Lys336, Arg350, Lys354 } \\
\text { Glu351 }\end{array}$ \\
\hline & (E)-4-Undecenal & 5283357 & -4.9 & x 2.075 & $x 40$ & Tyr169 & Leu167, Tyr192, Lys336 \\
\hline & & & & y 31.910 & y 40 & & $\begin{array}{c}\text { Arg350, Glu351, } \\
\text { Asp337 }\end{array}$ \\
\hline & & & & z 18.503 & z 40 & & Gln193, Lys354, Tyr189 \\
\hline & Octanoic acid & 379 & -4.7 & x 2.075 & $x 40$ & $\begin{array}{l}\text { Arg202, } \\
\text { Leu167 }\end{array}$ & Asp337, Thr168, Tyr192 \\
\hline & & & & $\begin{array}{l}\text { y } 31.910 \\
\text { z } 18.503\end{array}$ & $\begin{array}{l}\text { y } 40 \\
\text { z } 40\end{array}$ & & $\begin{array}{c}\text { Tyr169, Gln193, Tyr189 } \\
\text { Glu351, Lys336 }\end{array}$ \\
\hline & 9-Octadecenoic acid & 965 & -4.1 & x 2.075 & $x 40$ & $\begin{array}{l}\text { Arg164, } \\
\text { Glu208 }\end{array}$ & Glu207, Glu203, Asp166 \\
\hline & & & & y 31.910 & y 40 & & Lys336, Arg202, Val372 \\
\hline \multirow{8}{*}{$\begin{array}{l}\text { Positive } \\
\text { control }\end{array}$} & & & & z 18.503 & $\mathrm{z} 40$ & & Asn375, Val163 \\
\hline & Methyl palmitate & 8181 & -3.8 & x 2.075 & $x 40$ & $\begin{array}{l}\text { Glu291, } \\
\text { Aro288 }\end{array}$ & Glu343, Leu333, Leu330 \\
\hline & & & & $\begin{array}{l}\text { y } 31.910 \\
\text { z } 18.503\end{array}$ & $\begin{array}{l}\text { y } 40 \\
\mathrm{z} 40\end{array}$ & & $\begin{array}{c}\text { Leu228, Met329, Ala292 } \\
\text { Ile326, Glu295 }\end{array}$ \\
\hline & (c) Pioglitazone & 4829 & -7.7 & x 2.075 & $x 40$ & Arg288 & Ile326, Leu333, Met329 \\
\hline & & & & $\begin{array}{l}\text { y } 31.910 \\
\text { z } 18.503\end{array}$ & $\begin{array}{l}\text { y } 40 \\
\mathrm{z} 40\end{array}$ & & $\begin{array}{l}\text { Ala292, Ile341, Cys285 } \\
\text { Ser342, Glu343, Glu295 } \\
\text { Leu228 }\end{array}$ \\
\hline & (c) Rosiglitazone & 77999 & -7.4 & x 2.075 & $x 40$ & Tyr169 & Glu351, Tyr189, Gln193 \\
\hline & & & & $\begin{array}{l}\text { y } 31.910 \\
\text { z } 18.503\end{array}$ & $\begin{array}{l}\text { y } 40 \\
\text { z } 40\end{array}$ & & $\begin{array}{l}\text { Thr168, Leu167, Glu369 } \\
\text { Lys373, Val372, Arg350 }\end{array}$ \\
\hline & (c) Lobeglitazone & 9826451 & -7.3 & $\begin{array}{l}\text { x } 2.075 \\
\text { y } 31.910 \\
\text { z } 18.503\end{array}$ & $\begin{array}{l}x 40 \\
\text { y } 40 \\
\mathrm{z} 40\end{array}$ & Arg234 & $\begin{array}{l}\text { Val372, Asn375, Met334 } \\
\text { Val163, Lys230, Glu203 } \\
\text { Lys157, Val205, Arg164 } \\
\text { Arg202, Asp166, Lys336 }\end{array}$ \\
\hline \multirow[t]{13}{*}{$\begin{array}{l}\text { FABP3 } \\
\text { (PDB ID: } \\
\text { 5HZ9) }\end{array}$} & $(\star)$ Monoolein & 5283468 & -8.9 & $x-1.215$ & $x 40$ & $\begin{array}{l}\text { Arg31, } \\
\text { Thr57, } \\
\text { Phe58 }\end{array}$ & Ala29, Gln32, Phe28 \\
\hline & & & & $\begin{array}{c}y 46.730 \\
z-15.099\end{array}$ & $\begin{array}{l}\text { y } 40 \\
\text { z } 40\end{array}$ & & Gly27 \\
\hline & Glyceryl palmitate & 14900 & -8.7 & $\begin{array}{c}x-1.215 \\
y 46.730 \\
z-15.099\end{array}$ & $\begin{array}{l}240 \\
\times 40 \\
\text { y } 40\end{array}$ & $\operatorname{Arg} 31$ & $\begin{array}{l}\text { Thr57, Phe58, Ala29 } \\
\text { Gln32, Phe28, Lys22 }\end{array}$ \\
\hline & Nonadecanoic acid & 12591 & -8.3 & $\begin{array}{c}x-1.215 \\
y 46.730 \\
z-15.099\end{array}$ & $\begin{array}{l}240 \\
\times 40 \\
\mathrm{y} 40\end{array}$ & Lys22 & $\begin{array}{l}\text { Ala29, Gln32, Phe28 } \\
\text { Gly27, Gly25 }\end{array}$ \\
\hline & Stearic acid & 5281 & -8.3 & $\begin{array}{l}z-15.099 \\
x-1.215 \\
y 46.730\end{array}$ & $\begin{array}{l}\mathrm{Z} 40 \\
\times 40 \\
\mathrm{y} 40\end{array}$ & $\mathrm{n} / \mathrm{a}$ & $\begin{array}{l}\text { Gly27, Asp78, Thr122 } \\
\text { Phe58, Ala29, Phe28 }\end{array}$ \\
\hline & & & & $z-15.099$ & z 40 & & $\begin{array}{l}\text { Lys22, Lys59, Asp77 } \\
\text { Thr30 }\end{array}$ \\
\hline & $\begin{array}{l}\text { 2-Dodecen-1- } \\
\text { ylsuccinic } \\
\text { anhydride }\end{array}$ & 5362708 & -7.8 & $x-1.215$ & $x 40$ & $\begin{array}{l}\text { Thr30, } \\
\text { Gly27 }\end{array}$ & Gly25, Gln32, Ala29 \\
\hline & & & & $\begin{array}{c}y 46.730 \\
z-15.099\end{array}$ & $\begin{array}{l}\text { y } 40 \\
\mathrm{z} 40\end{array}$ & & Phe28 \\
\hline & Heptadec-8-ene & 520230 & -7.6 & $\begin{array}{c}x-1.215 \\
y 46.730 \\
z-15.099\end{array}$ & $\begin{array}{l}x 40 \\
\text { y } 40 \\
\text { z } 40\end{array}$ & $\mathrm{n} / \mathrm{a}$ & $\begin{array}{l}\text { Phe28, Met36, Thr57 } \\
\text { Val33, Ala29, Gln32 }\end{array}$ \\
\hline & $\begin{array}{l}\text { 2-Methyl-Z,Z-3,13- } \\
\text { octadecadienol }\end{array}$ & 5364412 & -7.4 & $x-1.215$ & $x 40$ & $\mathrm{n} / \mathrm{a}$ & Lys22, Gly25, Gly27 \\
\hline & & & & $\begin{array}{c}y 46.730 \\
z-15.099\end{array}$ & $\begin{array}{l}\text { y } 40 \\
\text { z } 40\end{array}$ & & Gln32, Phe28, Ala29 \\
\hline & 2-Tetradecynoic acid & 324386 & -7.4 & $x-1.215$ & $x 40$ & $\begin{array}{l}\text { Arg31, } \\
\text { Lvs22 }\end{array}$ & Phe58, Ala29, Gln32 \\
\hline & & & & $\begin{array}{c}y 46.730 \\
z-15.099\end{array}$ & $\begin{array}{l}\text { y } 40 \\
\text { z } 40\end{array}$ & & Phe28, Thr57, Lys59 \\
\hline
\end{tabular}


Table 7. Cont

\begin{tabular}{|c|c|c|c|c|c|c|c|}
\hline \multirow[b]{2}{*}{ Protein } & \multirow[b]{2}{*}{ Ligand } & \multirow[b]{2}{*}{$\begin{array}{l}\text { PubChem } \\
\text { ID }\end{array}$} & \multirow[b]{2}{*}{$\begin{array}{c}\text { Binding } \\
\text { Energy } \\
\text { (kcal/mol) }\end{array}$} & \multicolumn{2}{|c|}{ Grid Box } & \multirow{2}{*}{$\begin{array}{c}\begin{array}{c}\text { Hydrogen } \\
\text { Bond } \\
\text { Interactions }\end{array} \\
\text { Amino } \\
\text { Acid } \\
\text { Residue }\end{array}$} & \multirow{2}{*}{$\begin{array}{c}\begin{array}{c}\text { Hydrophobic } \\
\text { Interactions }\end{array} \\
\begin{array}{c}\text { Amino Acid } \\
\text { Residue }\end{array}\end{array}$} \\
\hline & & & & Center & Dimension & & \\
\hline & Methyl palmitate & 8181 & -7.1 & $\begin{array}{c}x-1.215 \\
y 46.730 \\
z-15.099\end{array}$ & $\begin{array}{l}\mathrm{x} 40 \\
\mathrm{y} 40 \\
\mathrm{z} 40\end{array}$ & $\mathrm{n} / \mathrm{a}$ & $\begin{array}{l}\text { Phe28, Gly27, Gly25 } \\
\text { Gln32, Ala29 }\end{array}$ \\
\hline & 9-Octadecenoic acid & 637517 & -7.1 & $\begin{array}{l}z-10.099 \\
x-1.215 \\
y 46.730\end{array}$ & $\begin{array}{l}Z 40 \\
\times 40 \\
\text { y } 40\end{array}$ & $\mathrm{n} / \mathrm{a}$ & $\begin{array}{l}\text { Ala29, Gly25, Phe28 } \\
\text { Gly27, Gly25, Gln32 }\end{array}$ \\
\hline & Myristic acid & 11005 & -6.9 & $\begin{array}{l}x-1.215 \\
y 46.730\end{array}$ & $\begin{array}{l}240 \\
\times 40 \\
\mathrm{y} 40\end{array}$ & Phe58 & $\begin{array}{l}\text { Ala29, Phe28, Gly27 } \\
\text { Lys22 }\end{array}$ \\
\hline & 2-Dodecenoic acid & 5282729 & -6.8 & $\begin{array}{c}z-15.099 \\
x-1.215 \\
y-46.730 \\
z-15.099\end{array}$ & $\begin{array}{l}\mathrm{z} 40 \\
\times 40 \\
\mathrm{y} 40 \\
\mathrm{z} 40\end{array}$ & Arg31 & $\begin{array}{l}\text { Lys59, Phe58, Thr57 } \\
\text { Ala29, Gln32, Phe28 } \\
\text { Lys22 }\end{array}$ \\
\hline & Citronellic acid & 10402 & -6.6 & $\begin{array}{c}x-1.215 \\
y 46.730 \\
z-15.099\end{array}$ & $\begin{array}{l}x 40 \\
\text { y } 40 \\
740\end{array}$ & Phe58 & $\begin{array}{l}\text { Lys22, Met36, Ala29 } \\
\text { Thr57 }\end{array}$ \\
\hline & Palmitic acid & 985 & -6.5 & $\begin{array}{l}\mathrm{x}-1.215 \\
\mathrm{y} 46.730\end{array}$ & $\begin{array}{l}240 \\
\times 40 \\
\text { y } 40\end{array}$ & $\mathrm{n} / \mathrm{a}$ & $\begin{array}{l}\text { Val33, Thr57, Lys22 } \\
\text { Phe58, Ala29, Gln32 }\end{array}$ \\
\hline & (E)-4-Undecenal & 5283357 & -6.5 & $\begin{array}{c}z-15.099 \\
x-1.215 \\
y-46.730\end{array}$ & $\begin{array}{l}\text { z } 40 \\
\text { x } 40 \\
\text { y } 40\end{array}$ & $\mathrm{n} / \mathrm{a}$ & $\begin{array}{l}\text { Gln32, Ala29, Gly25 } \\
\text { Gly27, Phe28 }\end{array}$ \\
\hline & Isopropyl hexanoate & 16832 & -6.4 & $\begin{array}{c}z-15.099 \\
x-1.215 \\
y 46.730\end{array}$ & $\begin{array}{l}\text { z } 40 \\
\times 40 \\
\text { y } 40\end{array}$ & $\mathrm{n} / \mathrm{a}$ & Ala29, Phe28, Val33 \\
\hline & Octane & 356 & -6.1 & $\begin{array}{c}z-15.099 \\
x-1.215 \\
y 46.730 \\
z-15.099\end{array}$ & $\begin{array}{l}\mathrm{z} 40 \\
\mathrm{x} 40 \\
\mathrm{y} 40 \\
\mathrm{z} 40\end{array}$ & $\mathrm{n} / \mathrm{a}$ & Phe28 \\
\hline & $\begin{array}{c}\text { 10-Bromoundecanoic } \\
\text { acid }\end{array}$ & 543401 & -6.1 & $x-1.215$ & $\times 40$ & $\mathrm{n} / \mathrm{a}$ & Thr57, Ala29, Gln32 \\
\hline & & & & $\begin{array}{c}y 46.730 \\
z-15.099\end{array}$ & $\begin{array}{l}\text { y } 40 \\
\text { z } 40\end{array}$ & & Phe28, Lys22, Thr57 \\
\hline & Nonanoic acid & 8158 & -6.0 & $\begin{array}{l}x-1.215 \\
y 46.730\end{array}$ & $\begin{array}{l}\times 40 \\
\text { y } 40\end{array}$ & $\mathrm{n} / \mathrm{a}$ & Gln32, Phe28 \\
\hline & Octanoic acid & 379 & -5.8 & $\begin{array}{c}z-15.099 \\
x-1.215 \\
y 46.730 \\
z-15.099\end{array}$ & $\begin{array}{l}y 40 \\
\mathrm{x} 40 \\
\mathrm{y} 40 \\
\mathrm{z} 40\end{array}$ & $\mathrm{n} / \mathrm{a}$ & $\begin{array}{l}\text { Gln32, Val33, Gly25 } \\
\text { Gly27, Phe28, Ala29 }\end{array}$ \\
\hline & $\begin{array}{c}1,3,4,5- \\
\text { Tetrahydroxycyclohe- } \\
\text { xanecarboxylic } \\
\text { acid }\end{array}$ & 1064 & -5.6 & $x-1.215$ & $x 40$ & $\begin{array}{l}\text { Asn307, } \\
\text { Ala306, } \\
\text { Lys229 }\end{array}$ & Val305, Thr252, Trp228 \\
\hline & Citronellol & 8842 & -5.4 & $\begin{array}{c}y 46.730 \\
z-15.099 \\
x-1.215\end{array}$ & $\begin{array}{l}\text { y } 40 \\
\text { z } 40 \\
x 40\end{array}$ & $\mathrm{n} / \mathrm{a}$ & Ala306, Glu255, Asn307 \\
\hline \multirow{7}{*}{$\begin{array}{l}\text { MMP1 } \\
\text { (PDB ID: } \\
\text { 1SU3) }\end{array}$} & & & & $\begin{array}{c}y 46.730 \\
z-15.099\end{array}$ & $\begin{array}{l}\text { y } 40 \\
\text { z } 40\end{array}$ & \multirow[b]{2}{*}{$\begin{array}{l}\text { Thr54, } \\
\text { Arg107, } \\
\text { His94 }\end{array}$} & $\begin{array}{l}\text { Thr252, Trp228, Arg248 } \\
\text { Gln230, Lys229 }\end{array}$ \\
\hline & $\begin{array}{l}3- \\
\text { Hydroxycyclohexanone }\end{array}$ & 439950 & -5.3 & $x-1.215$ & $\times 40$ & & Thr61, Leu52, Ile63 \\
\hline & Neral & 643779 & -5.0 & $\begin{array}{c}y 46.730 \\
z-15.099 \\
x-1.215 \\
y 46.730 \\
z-15.099\end{array}$ & $\begin{array}{l}\text { y } 40 \\
\text { z } 40 \\
x 40 \\
\text { y } 40 \\
\text { z } 40\end{array}$ & $\mathrm{n} / \mathrm{a}$ & $\begin{array}{c}\text { Glu73, Leu105 } \\
\text { Arg361, Thr411, Tyr441 } \\
\text { Met440 }\end{array}$ \\
\hline & Citronellal & 7794 & -4.9 & $\begin{array}{c}x-1.215 \\
y 46.730 \\
z-15.099\end{array}$ & $\begin{array}{l}x 40 \\
\text { y } 40 \\
\text { z } 40\end{array}$ & $\mathrm{n} / \mathrm{a}$ & $\begin{array}{l}\text { Thr411, Met440, Pro362 } \\
\text { Asp360, Val410, Tyr284 }\end{array}$ \\
\hline & $\begin{array}{c}(\star) \text { 2-Propenoic acid, } \\
\text { 3-phenyl-, methyl } \\
\text { ester }\end{array}$ & 7644 & -5.0 & x 34.394 & $x 40$ & $\begin{array}{l}\text { Arg399, } \\
\text { Tyr411, } \\
\text { Tyr397 }\end{array}$ & Asp418, Phe436, Tyr390 \\
\hline & Geranyl acetate & 1549026 & -4.5 & $\begin{array}{c}y-44.313 \\
\mathrm{z} 37.396 \\
x 34.394 \\
y-44.313 \\
\mathrm{z} 37.396\end{array}$ & $\begin{array}{l}\text { y } 40 \\
\text { z } 40 \\
x 40 \\
\text { y } 40 \\
\text { z } 40\end{array}$ & Tyr411 & $\begin{array}{c}\text { Phe419, Phe447, Pro449 } \\
\text { Lys413 } \\
\text { Arg399, Asp418, Pro449 } \\
\text { Phe447, Lys452, Phe419 } \\
\text { Tyr390, Tyr397 }\end{array}$ \\
\hline & 2-Dodecenoic acid & 5282729 & -4.3 & x 34.394 & $x 40$ & $\begin{array}{l}\text { Tyr397, } \\
\text { Tyr411 }\end{array}$ & Lys413, Tyr390, Pro449 \\
\hline
\end{tabular}


Table 7. Cont

\begin{tabular}{|c|c|c|c|c|c|c|c|}
\hline \multirow[b]{2}{*}{ Protein } & \multirow[b]{2}{*}{ Ligand } & \multirow[b]{2}{*}{$\begin{array}{l}\text { PubChem } \\
\text { ID }\end{array}$} & \multirow[b]{2}{*}{$\begin{array}{c}\text { Binding } \\
\text { Energy } \\
\text { (kcal/mol) }\end{array}$} & \multicolumn{2}{|c|}{ Grid Box } & \multirow{2}{*}{$\begin{array}{c}\begin{array}{c}\text { Hydrogen } \\
\text { Bond } \\
\text { Interactions }\end{array} \\
\text { Amino } \\
\text { Acid } \\
\text { Residue }\end{array}$} & \multirow{2}{*}{$\begin{array}{c}\begin{array}{c}\text { Hydrophobic } \\
\text { Interactions }\end{array} \\
\begin{array}{c}\text { Amino Acid } \\
\text { Residue }\end{array}\end{array}$} \\
\hline & & & & Center & Dimension & & \\
\hline \multirow{8}{*}{$\begin{array}{l}\text { Positive } \\
\text { control }\end{array}$} & & & & $\begin{array}{l}y-44.313 \\
z 37.396\end{array}$ & $\begin{array}{l}\text { y } 40 \\
\mathrm{z} 40\end{array}$ & \multirow{3}{*}{ Arg399 } & Phe419, Phe447, Asp418 \\
\hline & Citronellal & 7794 & -4.0 & $\begin{array}{l}2 \\
\times 34.394\end{array}$ & $\begin{array}{l}240 \\
\times 40\end{array}$ & & Phe419, Asp418, Tyr397 \\
\hline & & & & $\begin{array}{c}y-44.313 \\
z 37.396\end{array}$ & $\begin{array}{l}\text { y } 40 \\
\text { z } 40\end{array}$ & & $\begin{array}{l}\text { Phe436, Tyr390, Glu383 } \\
\text { Pro449 }\end{array}$ \\
\hline & (E)-4-Undecenal & 5283357 & -3.6 & x 34.394 & $\times 40$ & $\mathrm{n} / \mathrm{a}$ & Lys452, Asp418, Pro449 \\
\hline & & & & $y-44.313$ & $\begin{array}{l}\text { y } 40 \\
\text { z } 40\end{array}$ & \multirow{3}{*}{$\begin{array}{l}\text { Thr112, } \\
\text { His113, } \\
\text { Lys396 } \\
\text { Thr373 }\end{array}$} & $\begin{array}{c}\text { Phe436, Phe419, Phe447 } \\
\text { Tyr390. Tyr397 }\end{array}$ \\
\hline & (d) Batimastat & 5362422 & -6.7 & x 34.394 & $\times 40$ & & Pro146, Glu110, Arg108 \\
\hline & & & & $\begin{array}{l}\mathrm{y}-44.313 \\
\mathrm{z} 37.396\end{array}$ & $\begin{array}{l}\text { y } 40 \\
\text { z } 40\end{array}$ & & $\begin{array}{c}\text { Pro371, Trp398, Pro412 } \\
\text { Val393 }\end{array}$ \\
\hline & (d) Ilomastat & 132519 & -6.5 & $\begin{array}{c}x 34.394 \\
y-44.313 \\
z 37.396\end{array}$ & $\begin{array}{l}x 40 \\
\text { y } 40 \\
\text { z } 40\end{array}$ & His113 & $\begin{array}{c}\text { Thr145, Ser142, Thr148 } \\
\text { Leu147, Lys413, His417 } \\
\text { Met414, Pro412, Gln264 } \\
\text { Pro146 }\end{array}$ \\
\hline
\end{tabular}

${ }^{\star}{ }^{\star}$ Compound with the greatest affinity on each target; ${ }^{(a)}$ PPARA agonist; ${ }^{(b)}$ PPARD agonist; ${ }^{(c)}$ PPARG agonist; ${ }^{\text {(d) }}$ MMP agonist.

Table 8. Toxicological properties of seven key compounds in the MDT.

\begin{tabular}{|c|c|c|c|c|c|c|c|}
\hline \multirow[t]{2}{*}{ Parameters } & \multicolumn{7}{|c|}{ Compound Name } \\
\hline & Campesterol & $\begin{array}{c}26,27- \\
\text { Dinorergosta- } \\
\text { 5,23-dien-3 } \beta \text {-ol }\end{array}$ & $\begin{array}{c}\text { CBMicro } \\
013618\end{array}$ & Monoolein & $\begin{array}{c}\beta- \\
\text { Caryophyllene }\end{array}$ & $\begin{array}{l}\text { Stigmasta- } \\
\text { 5,22-dien-3-ol }\end{array}$ & NSC0402953 \\
\hline Ames toxicity & NAT & NAT & NAT & NAT & NAT & NAT & NAT \\
\hline Carcinogens & $\mathrm{NC}$ & $\mathrm{NC}$ & $\mathrm{NC}$ & $\mathrm{NC}$ & $\mathrm{NC}$ & $\mathrm{NC}$ & $\mathrm{NC}$ \\
\hline $\begin{array}{l}\text { Acute oral } \\
\text { toxicity }\end{array}$ & I & I & III & IV & III & I & III \\
\hline $\begin{array}{l}\text { Rat acute } \\
\text { toxicity }\end{array}$ & 2.8078 & 2.8078 & 2.5735 & 1.0526 & 1.4345 & 2.6561 & 1.9796 \\
\hline
\end{tabular}

AT: Ames toxic; NAT: Non-ames toxic; NC: Non-carcinogenic; category I means (50 mg/kg $\leq$ LD50); category II means (50 mg/kg > LD50 < $500 \mathrm{mg} / \mathrm{kg}$ ); category III means ( $500 \mathrm{mg} / \mathrm{kg}>$ LD50 < $5000 \mathrm{mg} / \mathrm{kg}$ ); category IV means (LD50 > 5000mg $/ \mathrm{kg}$ ).

\section{Discussion}

PPI network showed that the therapeutic efficacy of ZPFs on RA was associated with 99 targets. The KEGG pathway analysis enrichment of 99 targets revealed that 19 signaling pathways were related closely to the occurrence and progression of RA, suggesting that these signaling pathways might be remedial mechanisms of ZPFs to alleviate RA. The relationships of the 19 signaling pathways with RA are briefly discussed as follows. PPAR signaling pathway: the activation of PPAR is a good strategy to alleviate RA, which can suppress the inflammatory activity of NF-KB in fibroblast-like synoviocytes (FLSs) [30]. Relaxin signaling pathway: the combined relaxin with estrogen exerts anti-inflammatory effects by dampening neutrophil function [31]. Vascular Endothelial Growth Factor (VEGF) signaling pathway: VEGF expression level in RA patients increased significantly, compared with healthy groups; moreover, patients under RA for an extended period exerted higher VEGF expression level in serum [32]. Estrogen signaling pathway: the estrogen treatment might have an inhibitory effect on RA symptoms or delay in the onset of disease, and estrogen has anti-inflammatory activity in an animal test of RA [33]. Fc epsilon RI signaling pathway: Fc epsilon RI signaling pathway is associated with inflammatory etiology, antigen-induced autoimmune reaction, and control of lipid metabolic pathway, and damage in human joint [34]. Prolactin signaling pathway: Prolactin collaborates with other proinflammatory factors to stimulate macrophages through prolactin receptors which might be a potential therapeutic target in RA [35]. Sphingolipid signaling pathway: Sphingolipid expression level in RA elevated in the serum sample, consistent with known 
roles of sphingolipids related to inflammation [36]. Cyclic AMP (cAMP) signaling pathway: stimuli-induced cAMP production can exert proinflammatory effects in RA [37]. It implies that the activation of the cAMP level might drive inflammatory responses. AGERAGE (the receptor for advanced glycation end products) signaling pathway in diabetic complications: the expression of RAGE increased in RA patients, and IL-17 and IL- $1 \beta$ triggered RAGE production [37]. It suggests that the inhibition of RAGE might be a good target for RA treatment. Hypoxia-Inducible Factor-1(HIF-1) signaling pathway: synovial hypoxia is characterized by RA patients, leading to inflammation, cartilage destruction, and oxidative impairment [38]. Ras-associated protein-1 (RAP1) signaling pathway: The downregulation of RAP1 in RA induces ongoing inflammation due to the overproduction of free radicals [39]. Thyroid hormone signaling pathway: joint damages in thyroid gland abnormalities are due to hypothyroidism, and thyroid hormone plays a vital role in antioxidant modulation, as indicated by in vivo and in vitro tests [40,41]. Phospholipase D signaling pathway: phospholipase D1 (PLD1) is a mediator to induce proinflammatory cytokines with a reduction of the regulatory $\mathrm{T}$ (Treg) cell and recruitment of Th17 cell in collagen-induced arthritis mice [42]. Gonadotropin-releasing hormone (GnRH) signaling pathway: GnRH is a potent substance to alleviate inflammation in RA patients with a high level of GnRH [43]. Ras signaling pathway: T cells in RA patients show the Ras signalling pathway's overactivation-related deeply to inflammation [44]. T cell receptor signaling pathway: T cell recruitment to the inflammatory sites can induce chronic inflammation and autoimmunity [45]. Phosphoinositide 3-kinase-Akt (PI3K-Akt) signaling pathway: a report demonstrated that PI3K-Akt signaling pathway was excessively activated, aggravating in overexpression Bcl-2, Mcl-1, and FLIP to result in unbalanced apoptosis of synovial cells, which is associated with occurrence and progression of RA [46,47]. Calcium signaling pathway: it was reported that the cytoplasmic calcium concentration in RA naïve $\mathrm{CD}^{+} \mathrm{T}$ cells is increased significantly [48]. It implies that the calcium signaling pathway is involved intensely in inflammatory responses.

MAPK signaling pathway: a study demonstrated that andrographolide with antiinflammatory activities has potent anti-RA efficacy, inhibiting MAPK pathway [49]. This report is consistent with our result via network pharmacology analysis.

To sum things up, the relationship between compounds and targets through the network pharmacology concept was clarified, and ligands with the most excellent affinity out of selective ligands in ZPFs were considered as potential therapeutic candidates against RA, compared with existing ligands via MDT. The PPI network suggested that VEGFA is the highest degree of value (42) on the MAPK signaling pathway. However, the affinity of ZPFs compounds on VEGFA was invalid $(>-6.0 \mathrm{kcal} / \mathrm{mol}$ ) [29]; furthermore, existing ligand (BAW2881: $-7.6 \mathrm{kcal} / \mathrm{mol}$ ) had better affinity than a compound (3,4-O-Isopropylidene-dgalactose: $-5.3 \mathrm{kcal} / \mathrm{mol}$ ) with the greatest affinity from ZPFs. The STC network indicated that PRKCA had the greatest degree of value (14) on the MAPK signaling pathway, and monoolein (PubChem ID: 5283468$)$ had the greatest affinity $(-6.7 \mathrm{kcal} / \mathrm{mol})$, which was superior to an existing ligand (sphingosine: $-5.5 \mathrm{kcal} / \mathrm{mol}$ ). On PPAR signaling pathway, $\beta$-Caryophyllene $(-8.6 \mathrm{kcal} / \mathrm{mol})$ on PPARA, Stigmasta-5,22-dien-3-ol $(-8.6 \mathrm{kcal} / \mathrm{mol})$ on PPARD, and NSC402953 $(-8.2 \mathrm{kcal} / \mathrm{mol})$ on PPARG had better affinity than existing ligands on each target. Specifically, monoolein on FABP3 had the greatest affinity $(-8.9 \mathrm{kcal} / \mathrm{mol})$. Moreover, agonists of FABP3 were not reported yet. Furthermore, there were no valid ligands on MMP1 on the PPAR signaling pathway. Taken together, the most noticeable ligand of ZPFs against RA is monoolein with dual effects on RA. Monoolein might be an antagonist on the MAPK signaling pathway or an agonist on the PPAR signaling pathway (Figure 10). A study demonstrated that monoolein treatment in LPS- stimulated primary murine bone marrow-derived dendritic cells (BMDCs) inhibited the activation of MAPK and NF- $\mathrm{kB}$; moreover, it suppressed the production of NO and iNOS in RAW264.7 cells [50]. This suggests that the inactivation of the MAPK signaling pathway is a good strategy to ameliorate inflammation associated with bone damage. Another report indicated that the expression level of PPARG in FLSs of RA patients was significantly reduced compared 
with healthy FLSs [30]; this implies that the activation of the PPAR signaling pathway might be a therapeutic mechanism against RA. These reports are in line with our results. Additionally, we suggested that monoolein might be an antagonist in activating PRKCA on MAPK signaling pathway and might also be an agonist to activate FABP3 on the PPAR signaling pathway.
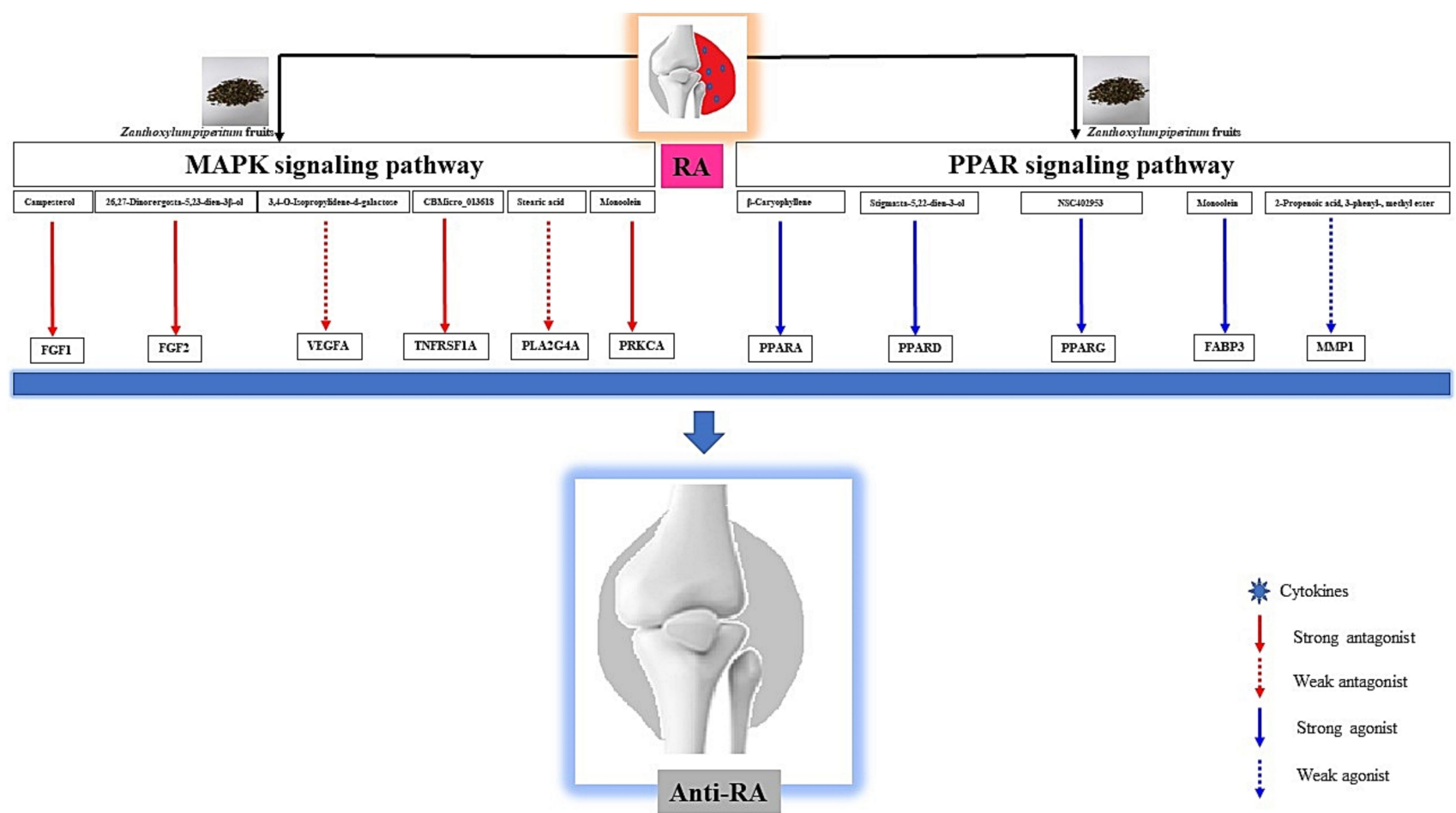

Figure 10. Schematic representation of key findings in the study.

\section{Conclusions}

This study provides eight targets, seven compounds, and two key signaling pathways of ZPFs which show promise against RA, which might be useful for multiple target combination therapies on RA. Out of seven compounds from ZPFs, monoolein shows dual effects: inactivation of MAPK signaling pathway and activation of the PPAR signaling pathway. The key pharmacological mechanisms of ZPFs against RA might be to inhibit cytokine production in synovial cells by binding on PRKCA or FABP3.

Supplementary Materials: The following are available online at https: / www.mdpi.com/article / 10.3390/biology10080703/s1, Table S1: The list of 470, 629, and 215 targets from SEA, STP, and overlapping target proteins between SEA and STP, respectively, Table S2: The list of 3377 target proteins associated with RA, Table S3: The number of final 101 target proteins of ZPFs on RA.

Author Contributions: Conceptualization, methodology, formal analysis, investigation, visualization, data curation, writing - original draft, K.O.; software, investigation, data curation, K.O. and M.A.; validation, writing-review and editing, M.A.; supervision, project administration, D.C. All authors have read and agreed to the published version of the manuscript.

Funding: This research did not receive any specific grant from funding agencies in the public, commercial, or not-for-profit sectors.

Institutional Review Board Statement: Not applicable.

Informed Consent Statement: Not applicable.

Data Availability Statement: All data generated or analyzed during this study are included in this published article (and its Supplementary Materials). 
Acknowledgments: This research was acknowledged by the Department of Bio-Health Convergence, College of Biomedical Science, Kangwon National University, Chuncheon 24341, Korea.

Conflicts of Interest: There is no conflict of interest declared.

$\begin{array}{ll}\text { Abbreviations } \\ \text { AGE } & \text { Advanced Glycation End-product; } \\ \text { AMPK } & \text { AMP-activated protein kinase; } \\ \text { cAMP } & \text { Cyclic AMP; } \\ \text { DMARDs } & \text { Disease-Modifying Arthritis Drugs; } \\ \text { FLSs } & \text { Fibroblast-Like Synoviocytes; } \\ \text { GC-MS } & \text { Gas Chromatography Mass Spectrum; } \\ \text { GnRH } & \text { Gonadotropin releasing hormone; } \\ \text { HIF-1 } & \text { Hypoxia Inducible Factor-1; } \\ \text { KEGG } & \text { Kyoto Encyclopedia of Genes and Genomes; } \\ \text { LPS } & \text { Lipopolysaccharide; } \\ \text { MAPK } & \text { Mitogen Activated Protein Kinase; } \\ \text { MDT } & \text { Molecular Docking Test; } \\ \text { NF-KB } & \text { Nuclear Factor Kappa B; } \\ \text { NSAIDs } & \text { Non-Steroidal Anti-Inflammatories Drugs; } \\ \text { OMIM } & \text { Online Mendelian Inheritance in Man; } \\ \text { PI3K-Akt } & \text { Phosphoinositide 3-kinase-Akt; } \\ \text { PLD } & \text { Phospholipase D; } \\ \text { PLD1 } & \text { Phospholipase D1; } \\ \text { PPAR } & \text { Peroxisome Proliferator Activated Receptor; } \\ \text { PPARA } & \text { Peroxisome Proliferator Activated Receptor Alpha; } \\ \text { PPARD } & \text { Peroxisome Proliferator Activated Receptor Delta; } \\ \text { PPARG } & \text { Peroxisome Proliferator Activated Receptor Gamma; } \\ \text { PPI } & \text { Protein-protein interaction; } \\ \text { PRKCA } & \text { Protein Kinase C Alpha; } \\ \text { RA } & \text { Rheumatoid Arthritis; } \\ \text { RAGE } & \text { The Receptor for Advanced Glycation End Products; } \\ \text { Rap1 } & \text { Ras-associated protein-1; } \\ \text { SEA } & \text { Similarity Ensemble Approach; } \\ \text { SMILES } & \text { Simplified Molecular Input Line Entry System; } \\ \text { STP } & \text { SwissTargetPrediction; } \\ \text { VEGF } & \text { Vascular Endothelial Growth Factor; } \\ \text { VEGFA } & \text { Vascular Endothelial Growth Factor A; } \\ \text { ZP } & \text { Zanthoxylum piperitum; } \\ \text { ZPFs } & \text { Zanthoxylum piperitum fruits } \\ & \end{array}$

\section{References}

1. Guo, Q.; Wang, Y.; Xu, D.; Nossent, J.; Pavlos, N.J.; Xu, J. Rheumatoid arthritis: Pathological mechanisms and modern pharmacologic therapies. Bone Res. 2018, 6, 1-14. [CrossRef] [PubMed]

2. Heidari, B. Rheumatoid arthritis: Early diagnosis and treatment outcomes. Casp. J. Intern. Med. 2011, 2, 161-170.

3. El Miedany, Y.; Youssef, S.; Mehanna, A.N.; El Gaafary, M. Development of a scoring system for assessment of outcome of early undifferentiated inflammatory synovitis. Jt. Bone Spine 2008, 75, 155-162. [CrossRef]

4. McInnes, I.B.; Schett, G. Pathogenetic insights from the treatment of rheumatoid arthritis. Lancet 2017, 389, 2328-2337. [CrossRef]

5. Noack, M.; Miossec, P. Selected cytokine pathways in rheumatoid arthritis. Semin. Immunopathol. 2017, 39, 365-383. [CrossRef] [PubMed]

6. Scherer, H.U.; Häupl, T.; Burmester, G.R. The etiology of rheumatoid arthritis. J. Autoimmun. 2020, 110, 102400. [CrossRef]

7. Kumar, P.; Banik, S. Pharmacotherapy options in rheumatoid arthritis. Clin. Med. Insights: Arthritis Musculoskelet. Disord. 2013, 6, 35-43. [CrossRef]

8. Grove, M.L.; Hassell, A.B.; Hay, E.M.; Shadforth, M.F. Adverse reactions to disease-modifying anti-rheumatic drugs in clinical practice. QJM Mon. J. Assoc. Physicians 2001, 94, 309-319. [CrossRef] [PubMed]

9. Wongrakpanich, S.; Wongrakpanich, A.; Melhado, K.; Rangaswami, J. A comprehensive review of non-steroidal anti-inflammatory drug use in the elderly. Aging Dis. 2018, 9, 143-150. [CrossRef] 
10. Hajja, G.; Bahlouli, A. Medicinal plants in the prevention and treatment of rheumatoid arthritis. MOJ Bioequivalence Bioavailab. 2018, 5. [CrossRef]

11. Farzaei, M.H.; Farzaei, F.; Abdollahi, M.; Abbasabadi, Z.; Abdolghaffari, A.H.; Mehraban, B. A mechanistic review on medicinal plants used for rheumatoid arthritis in traditional Persian medicine. J. Pharm. Pharmacol. 2016, 68, 1233-1248. [CrossRef]

12. Chung, M.-S. Volatile Compounds of Zanthoxylum piperitum A.P. DC. Food Sci. Biotechnol. 2005, 14, 529-532.

13. Lee, J.-H.; Chang, K.-M.; Kim, G.-H. Anti-inflammatory Activities of Chopi (Zanthoxylum piperitum A.P. DC) Essential Oil: Suppression of the Inducible Nitric Oxide Synthase and Cellular Adhesion. Food Sci. Biotechnol. 2009, 18, 1371-1378.

14. Hee, C.Y.; Yil, M.N. The Anti-inflammatory Mechanism of the Peel of Zanthoxylum piperitum D.C. is by Suppressing NFkB/Caspase-1 Activation in LPS-Induced RAW264.7 Cells. Korean J. Plant Reources. 2019, 32, 669-676.

15. Oh, K.K.; Adnan, M.; Cho, D.H. Network pharmacology of bioactives from Sorghum bicolor with targets related to diabetes mellitus. PLoS ONE 2020, 15, e0240873. [CrossRef]

16. Oh, K.K.; Adnan, M.; Cho, D.H. Network pharmacology approach to decipher signaling pathways associated with target proteins of NSAIDs against COVID-19. Sci. Rep. 2021, 11, 9606. [CrossRef]

17. Oh, K.-K.; Adnan, M.; Cho, D.-H. Network Pharmacology Study to Interpret Signaling Pathways of Ilex cornuta Leaves against Obesity. Processes 2021, 9, 1106. [CrossRef]

18. Chandran, U.; Mehendale, N.; Patil, S.; Chaguturu, R.; Patwardhan, B. Network Pharmacology. In Innovative Approaches in Drug Discovery: Ethnopharmacology, Systems Biology and Holistic Targeting; Elsevier Inc.: Amsterdam, The Netherlands, 2017; pp. 127-164, ISBN 9780128018224.

19. Lai, X.; Wang, X.; Hu, Y.; Su, S.; Li, W.; Li, S. Editorial: Network Pharmacology and Traditional Medicine. Front. Pharmacol. 2020, 11, 1194. [CrossRef]

20. Oh, K.K.; Adnan, M.; Ju, I.; Cho, D.H. A network pharmacology study on main chemical compounds fromHibiscus cannabinusL. leaves. RSC Adv. 2021, 11, 11062-11082. [CrossRef]

21. Keiser, M.J.; Roth, B.L.; Armbruster, B.N.; Ernsberger, P.; Irwin, J.J.; Shoichet, B.K. Relating protein pharmacology by ligand chemistry. Nat. Biotechnol. 2007, 25, 197-206. [CrossRef]

22. Daina, A.; Michielin, O.; Zoete, V. SwissTargetPrediction: Updated data and new features for efficient prediction of protein targets of small molecules. Nucleic Acids Res. 2019, 47, W357-W3664. [CrossRef] [PubMed]

23. Szklarczyk, D.; Gable, A.L.; Lyon, D.; Junge, A.; Wyder, S.; Huerta-Cepas, J.; Simonovic, M.; Doncheva, N.T.; Morris, J.H.; Bork, P.; et al. STRING v11: Protein-protein association networks with increased coverage, supporting functional discovery in genome-wide experimental datasets. Nucleic Acids Res. 2019, 47, D607-D613. [CrossRef]

24. Khanal, P.; Patil, B.M.; Chand, J.; Naaz, Y. Anthraquinone Derivatives as an Immune Booster and their Therapeutic Option Against COVID-19. Nat. Prod. Bioprospecting 2020, 10, 325-335. [CrossRef] [PubMed]

25. Kanehisa, M.; Furumichi, M.; Sato, Y.; Ishiguro-Watanabe, M.; Tanabe, M. KEGG: Integrating viruses and cellular organisms. Nucleic Acids Res. 2021, 49, D545-D551. [CrossRef]

26. Barton, A.; Woolmore, J.A.; Ward, D.; Eyre, S.; Hinks, A.; Ollier, W.E.R.; Strange, R.C.; Fryer, A.A.; John, S.; Hawkins, C.P.; et al. Association of protein kinase C alpha (PRKCA) gene with multiple sclerosis in a UK population. Brain 2004, 127, 1717-1722. [CrossRef] [PubMed]

27. Makino, H.; Seki, S.; Yahara, Y.; Shiozawa, S.; Aikawa, Y.; Motomura, H.; Nogami, M.; Watanabe, K.; Sainoh, T.; Ito, H.; et al. A selective inhibition of c-Fos/activator protein-1 as a potential therapeutic target for intervertebral disc degeneration and associated pain. Sci. Rep. 2017, 7, 1-14. [CrossRef]

28. Arias de la Rosa, I.; Escudero-Contreras, A.; Rodríguez-Cuenca, S.; Ruiz-Ponce, M.; Jiménez-Gómez, Y.; Ruiz-Limón, P.; PérezSánchez, C.; Ábalos-Aguilera, M.C.; Cecchi, I.; Ortega, R.; et al. Defective glucose and lipid metabolism in rheumatoid arthritis is determined by chronic inflammation in metabolic tissues. J. Intern. Med. 2018, 284, 61-77. [CrossRef]

29. Shityakov, S.; Förster, C. In silico predictive model to determine vector-mediated transport properties for the blood-brain barrier choline transporter. Adv. Appl. Bioinform. Chem. 2014, 7, 23-36. [CrossRef] [PubMed]

30. Liu, Y.; Wang, J.; Luo, S.; Zhan, Y.; Lu, Q. The roles of PPAR $\gamma$ and its agonists in autoimmune diseases: A comprehensive review. J. Autoimmun. 2020, 113, 102510. [CrossRef] [PubMed]

31. Yamasaki, S.; Nakashima, T.; Kawakami, A.; Miyashita, T.; Ida, H.; Migita, K.; Nakata, K.; Eguchi, K. Functional changes in rheumatoid fibroblast-like synovial cells through activation of peroxisome proliferator-activated receptor $\gamma$-mediated signalling pathway. Clin. Exp. Immunol. 2002, 129, 379-384. [CrossRef]

32. Szekanecz, Z.; Koch, A.E. VEGF as an activity marker in rheumatoid arthritis. Int. J. Clin. Rheumatol. 2010, 5, 287-289. [CrossRef]

33. Fernanda Romo-García, M.; Zapata-Zuñiga, M.; Antonio Enciso-Moreno, J.; Enrique Castañeda-Delgado, J. The Role of Estrogens in Rheumatoid Arthritis Physiopathology. In Rheumatoid Arthritis-Other Perspectives towards a Better Practice; IntechOpen: London, UK, 2020.

34. Huang, L.; Lv, Q.; Xie, D.; Shi, T.; Wen, C. Deciphering the Potential Pharmaceutical Mechanism of Chinese Traditional Medicine (Gui-Zhi-Shao-Yao-Zhi-Mu) on Rheumatoid Arthritis. Sci. Rep. 2016, 6, 1-12. [CrossRef]

35. Tang, M.W.; Reedquist, K.A.; Garcia, S.; Fernandez, B.M.; Codullo, V.; Vieira-Sousa, E.; Goffin, V.; Reuwer, A.Q.; Twickler, M.T.; Gerlag, D.M.; et al. The prolactin receptor is expressed in rheumatoid arthritis and psoriatic arthritis synovial tissue and contributes to macrophage activation. Rheumatology 2016, 55, 2248-2259. [CrossRef] 
36. Miltenberger-Miltenyi, G.; Cruz-Machado, A.R.; Saville, J.; Conceição, V.A.; Calado, Â.; Lopes, I.; Fuller, M.; Fonseca, J.E. Increased monohexosylceramide levels in the serum of established rheumatoid arthritis patients. Rheumatology 2020, 59, 2085-2089. [CrossRef]

37. Lorenowicz, M.J.; Fernandez-Borja, M.; Hordijk, P.L. cAMP signaling in leukocyte transendothelial migration. Arterioscler. Thromb. Vasc. Biol. 2007, 27, 1014-1022. [CrossRef]

38. Quiñonez-Flores, C.M.; González-Chávez, S.A.; Pacheco-Tena, C. Hypoxia and its implications in rheumatoid arthritis. J. Biomed. Sci. 2016, 23, 1-9. [CrossRef]

39. Remans, P.; Reedquist, K.; Bos, J.; Verweij, C.; Breedveld, F.; van Laar, J.; Gringhuis, S. Deregulated Ras and Rap1 signaling in rheumatoid arthritis T cells leads to persistent production of free radicals. Arthritis Res. Ther. 2002, 4, 1-38. [CrossRef]

40. Silman, A.J.; Ollier, W.E.R.; Bubel, M.A. Autoimmune thyroid disease and thyroid autoantibodies in rheumatoid arthritis patients and their families. Rheumatology 1989, 28, 18-21. [CrossRef]

41. Mancini, A.; Di Segni, C.; Raimondo, S.; Olivieri, G.; Silvestrini, A.; Meucci, E.; Currò, D. Thyroid Hormones, Oxidative Stress, and Inflammation. Mediat. Inflamm. 2016, 2016, 1-12. [CrossRef]

42. Yoo, H.J.; Hwang, W.C.; Min, D.S. Targeting of phospholipase d1 ameliorates collagen-induced arthritis via modulation of treg and Th17 cell imbalance and suppression of osteoclastogenesis. Int. J. Mol. Sci. 2020, 21, 3230. [CrossRef]

43. Kåss, A.; Hollan, I.; Fagerland, M.W.; Gulseth, H.C.; Torjesen, P.A.; Førre, Ø.T. Rapid anti-inflammatory effects of gonadotropinreleasing hormone antagonism in rheumatoid arthritis patients with high gonadotropin levels in the Agra trial. PLoS ONE 2015, 10. [CrossRef] [PubMed]

44. Zayoud, M.; Marcu-Malina, V.; Vax, E.; Jacob-Hirsch, J.; Elad-Sfadia, G.; Barshack, I.; Kloog, Y.; Goldstein, I. Ras signaling inhibitors attenuate disease in adjuvant-induced arthritis via targeting pathogenic antigen-specific Th17-type cells. Front. Immunol. 2017, 8, 799. [CrossRef]

45. Mellado, M.; Martínez-Muñoz, L.; Cascio, G.; Lucas, P.; Pablos, J.L.; Rodríguez-Frade, J.M. T cell migration in rheumatoid arthritis. Front. Immunol. 2015, 6, 384. [CrossRef]

46. Ito, K.; Caramori, G.; Adcock, I.M. Therapeutic potential of phosphatidylinositol 3-kinase inhibitors in inflammatory respiratory disease. J. Pharmacol. Exp. Ther. 2007, 321, 1-8. [CrossRef]

47. Smith, M.D.; Weedon, H.; Papangelis, V.; Walker, J.; Roberts-Thomson, P.J.; Ahern, M.J. Apoptosis in the rheumatoid arthritis synovial membrane: Modulation by disease-modifying anti-rheumatic drug treatment. Rheumatology 2010, 49, 862-875. [CrossRef]

48. Ye, Z.; Shen, Y.; Jin, K.; Qiu, J.; Hu, B.; Jadhav, R.R.; Sheth, K.; Weyand, C.M.; Goronzy, J.J. Arachidonic acid-regulated calcium signaling in $\mathrm{T}$ cells from patients with rheumatoid arthritis promotes synovial inflammation. Nat. Commun. 2021, 12, 1-17. [CrossRef]

49. Li, Z.Z.; Tan, J.P.; Wang, L.L.; Li, Q.H. Andrographolide Benefits Rheumatoid Arthritis via Inhibiting MAPK Pathways. Inflammation 2017, 40, 1599-1605. [CrossRef]

50. Ali, I.; Manzoor, Z.; Koo, J.E.; Moon, S.R.; Byeon, S.H.; Yoo, E.S.; Kang, H.K.; Hyun, J.W.; Lee, N.H.; Koh, Y.S. Monoolein, isolated from Ishige sinicola, inhibits lipopolysaccharide-induced inflammatory response by attenuating mitogen-activated protein kinase and NF-kB pathways. Food Sci. Biotechnol. 2017, 26, 507-511. [CrossRef] 